\title{
Far away from flatland. Synthesis and molecular structure of di- and tri-hetera[3.3.n]propellanes - advanced analogues of morpholine/piperazine
}

Yevhenii M. Sokolenko, Yevhen Yu. Yurov, Bohdan V. Vashchenko, Oleksandr V. Hryshchuk, Yuliia Filimonova, Eugeniy N. Ostapchuk, Artem Artemenko, Oleg V. Zaremba, Oleksandr O. Grygorenko*

\section{Table of contents}

cis-Hexahydro-3a,6a-(methanooxymethano)cyclopenta[c]pyrrol-2-ium chloride (15) ${ }^{1} \mathrm{H}$ NMR

cis-Hexahydro-3a,6a-(methanooxymethano)cyclopenta[c]pyrrol-2-ium chloride (15) ${ }^{13} \mathrm{C}$ NMR

Tetrahydro-1H-3a,6a-ethanofuro[3,4-c]pyrrol-5-ium chloride (16) ${ }^{1} \mathrm{H}$ NMR

Tetrahydro-1H-3a,6a-ethanofuro[3,4-c]pyrrol-5-ium chloride $(16){ }^{13} \mathrm{C}$ NMR

Tetrahydro-1H-3a,6a-(methanooxymethano)furo[3,4-c]pyrrol-5-ium chloride (17) ${ }^{1} \mathrm{H}$ NMR ......

Tetrahydro-1H-3a,6a-(methanooxymethano)furo[3,4-c]pyrrol-5-ium chloride $(17){ }^{13} \mathrm{C}$ NMR .

Hexahydro-3a,6a-(methanooxymethano)pyrrolo[3,4-c]pyrrole-2,5-diium chloride (18) ${ }^{1} \mathrm{H}$ NMR .

Hexahydro-3a,6a-(methanoiminomethano)pyrrolo[3,4-c] pyrrole-2,5-diium chloride (19) ${ }^{1} \mathrm{H}$ NMR

Hexahydro-3a,6a-(methanoiminomethano)pyrrolo[3,4-c]pyrrole-2,5-diium chloride (19) ${ }^{13} \mathrm{C}$ NMR. 
tert-Butyl tetrahydro-1H-3a,7a-(methanoiminomethano)isoindole-2(3H)-carboxylate $(22){ }^{13} \mathrm{C} \mathrm{NMR} \mathrm{.}$

tert-Butyl tetrahydro-3a,6a-(methanoiminomethano)cyclopenta[c]pyrrole-2(1H)-carboxylate $(23)^{1}{ }^{H}$ NMR .......................................................................2 21

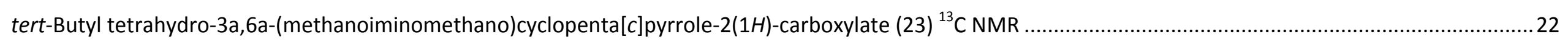

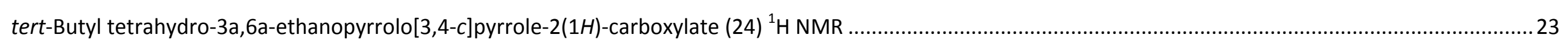

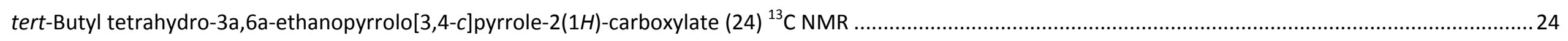

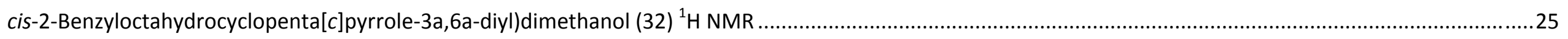

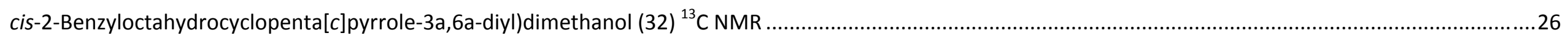

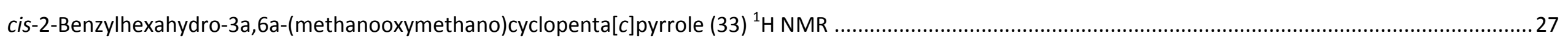

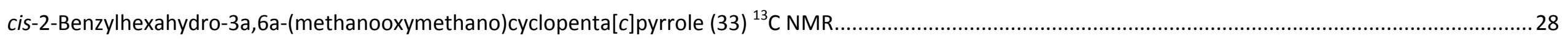

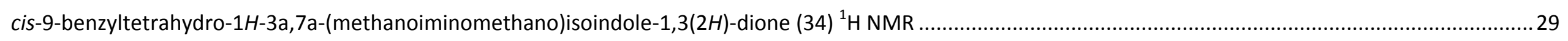

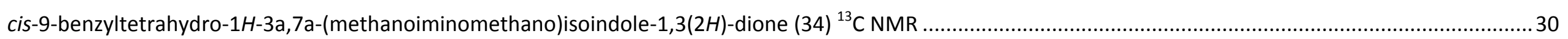

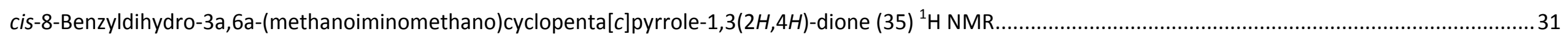

cis-8-Benzyldihydro-3a,6a-(methanoiminomethano) cyclopenta[c]pyrrole-1,3(2H,4H)-dione $(35){ }^{13} \mathrm{C}$ NMR ...........................................................................32

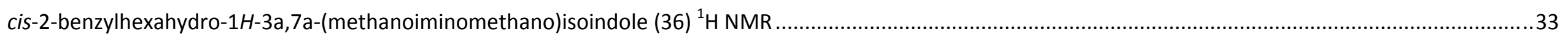

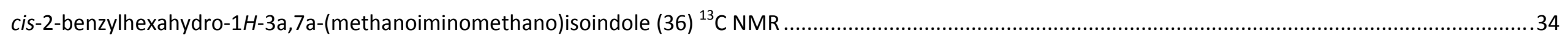

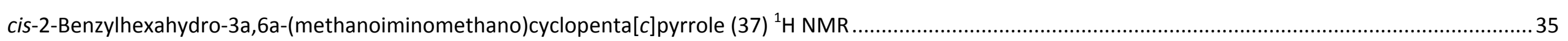

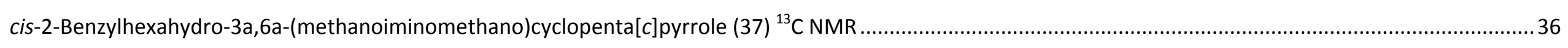

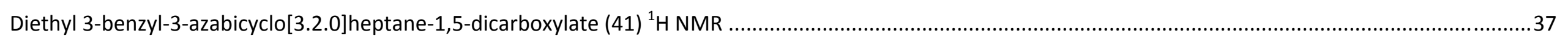

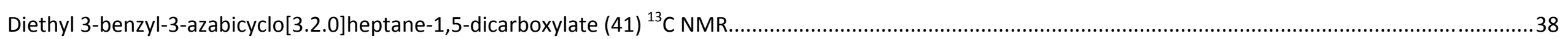

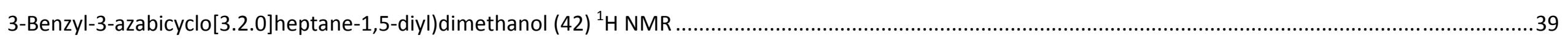

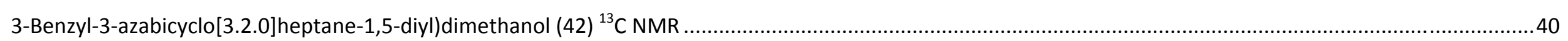

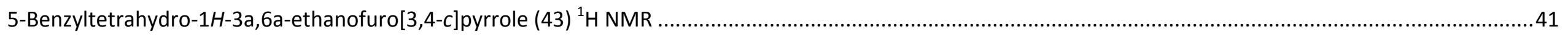


5-Benzyltetrahydro-1H-3a,6a-ethanofuro[3,4-c]pyrrole (43) ${ }^{13} \mathrm{C} N \mathrm{NMR}$

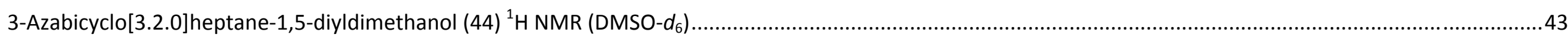

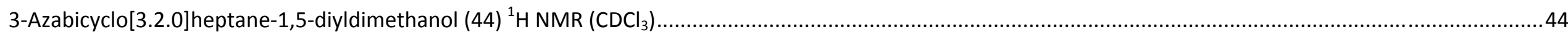

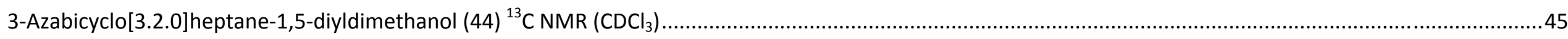

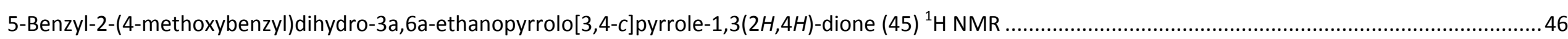

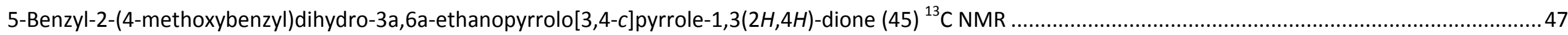

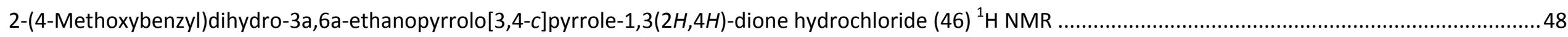

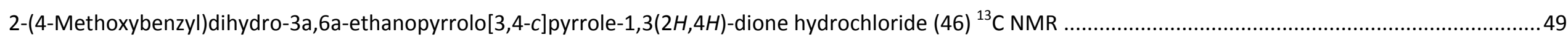

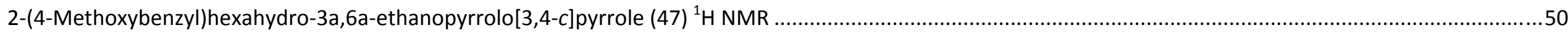

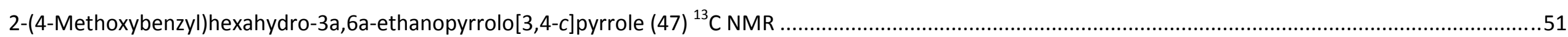

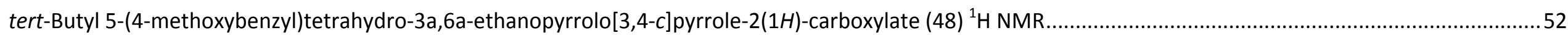

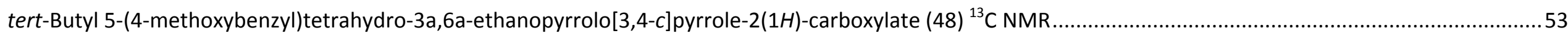

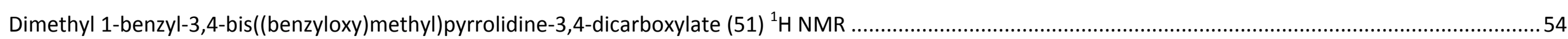

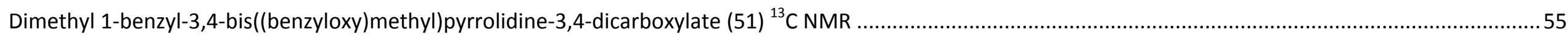

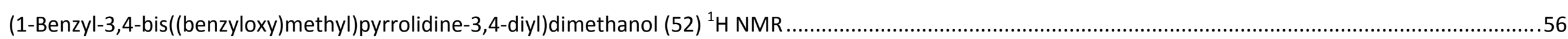

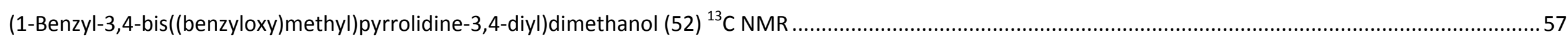

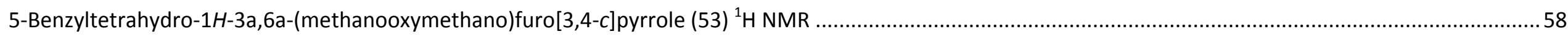

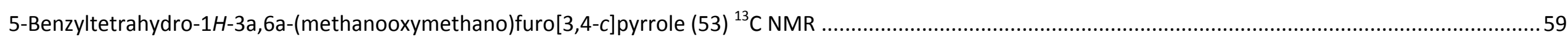

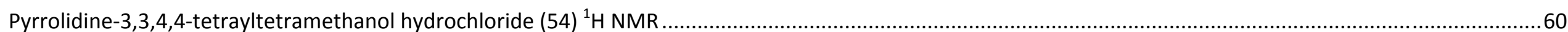

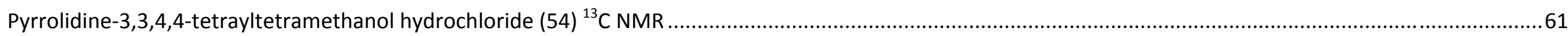

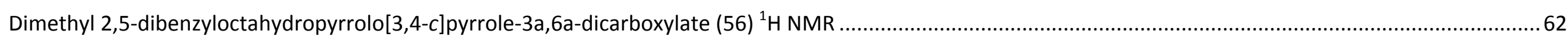

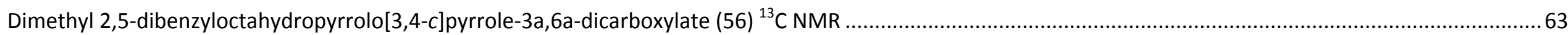


(2,5-Dibenzyloctahydropyrrolo[3,4-c]pyrrole-3a,6a-diyl)dimethanol (57) ${ }^{1} \mathrm{H}$ NMR .

(2,5-Dibenzyloctahydropyrrolo[3,4-c]pyrrole-3a,6a-diyl)dimethanol $(57){ }^{13} \mathrm{C}$ NMR.

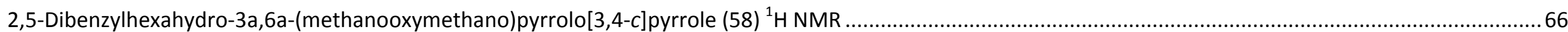

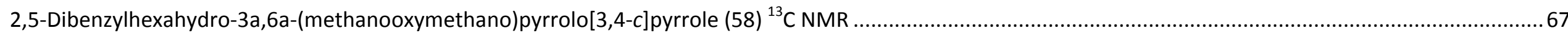

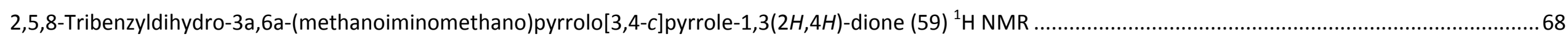

2,5,8-Tribenzyldihydro-3a,6a-(methanoiminomethano)pyrrolo[3,4-c]pyrrole-1,3(2H,4H)-dione $(59){ }^{13} \mathrm{C} \mathrm{NMR} \mathrm{.........................................................................69}$

2,5,8-Tribenzylhexahydro-3a,6a-(methanoiminomethano)pyrrolo[3,4-c]pyrrole (60) ${ }^{1} \mathrm{H}$ NMR............................................................................................. 70

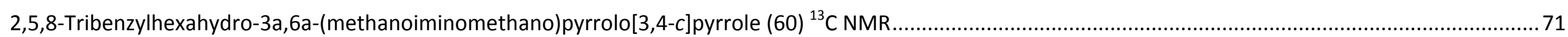

5,8-Dibenzyl-2-(4-methoxybenzyl)dihydro-3a,6a-(methanoiminomethano)pyrrolo[3,4-c]pyrrole-1,3(2H,4H)-dione (61) ${ }^{1} \mathrm{H}$ NMR ................................................72

5,8-Dibenzyl-2-(4-methoxybenzyl)dihydro-3a,6a-(methanoiminomethano)pyrrolo[3,4-c]pyrrole-1,3(2H,4H)-dione $(61){ }^{13} \mathrm{C} \mathrm{NMR} \mathrm{\ldots ...............................................} \mathrm{73}$

2,5-dibenzyl-8-(4-methoxybenzyl)hexahydro-3a,6a-(methanoiminomethano)pyrrolo[3,4-c]pyrrole $(62){ }^{1} \mathrm{H}$ NMR ..................................................................... 74

2,5-dibenzyl-8-(4-methoxybenzyl)hexahydro-3a,6a-(methanoiminomethano)pyrrolo[3,4-c]pyrrole $(62){ }^{13} \mathrm{C} \mathrm{NMR} \mathrm{.....................................................................75}$

cis-Hexahydro-1H-3a,7a-(methanooxymethano)isoindol-2-ium chloride (14 (A)) ORTEP diagram (Projection 1) .........................................................................76

cis-Hexahydro-1H-3a,7a-(methanooxymethano)isoindol-2-ium chloride (14 (A)) ORTEP diagram (Projection 2) .....................................................................77

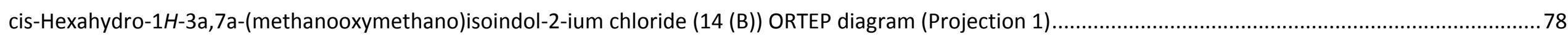

cis-Hexahydro-1H-3a,7a-(methanooxymethano)isoindol-2-ium chloride (14 (B)) ORTEP diagram (Projection 2)......................................................................... 79

cis-Hexahydro-3a,6a-(methanooxymethano)cyclopenta[c]pyrrol-2-ium chloride (15) ORTEP diagram (Projection 1) ................................................................8 80

cis-Hexahydro-3a,6a-(methanooxymethano)cyclopenta[c]pyrrol-2-ium chloride (15) ORTEP diagram (Projection 2) ...................................................................81

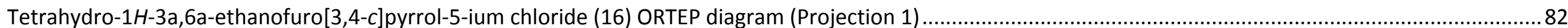

Tetrahydro-1H-3a,6a-ethanofuro[3,4-c]pyrrol-5-ium chloride (16) ORTEP diagram (Projection 2) ..................................................................................... 83

Hexahydro-3a,6a-(methanooxymethano)pyrrolo[3,4-c]pyrrole-2,5-diium chloride (18 (A)) ORTEP diagram (Projection 1) .........................................................8 84

Hexahydro-3a,6a-(methanooxymethano)pyrrolo[3,4-c]pyrrole-2,5-diium chloride (18 (A)) ORTEP diagram (Projection 2) ……....................................................85 
Hexahydro-3a,6a-(methanooxymethano)pyrrolo[3,4-c]pyrrole-2,5-diium chloride (18 (B)) ORTEP diagram (Projection 1).

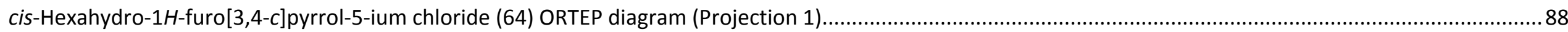

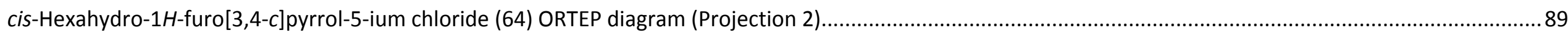

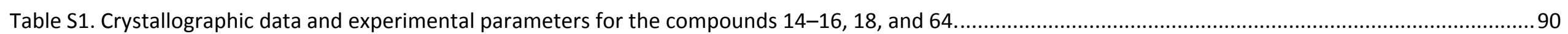



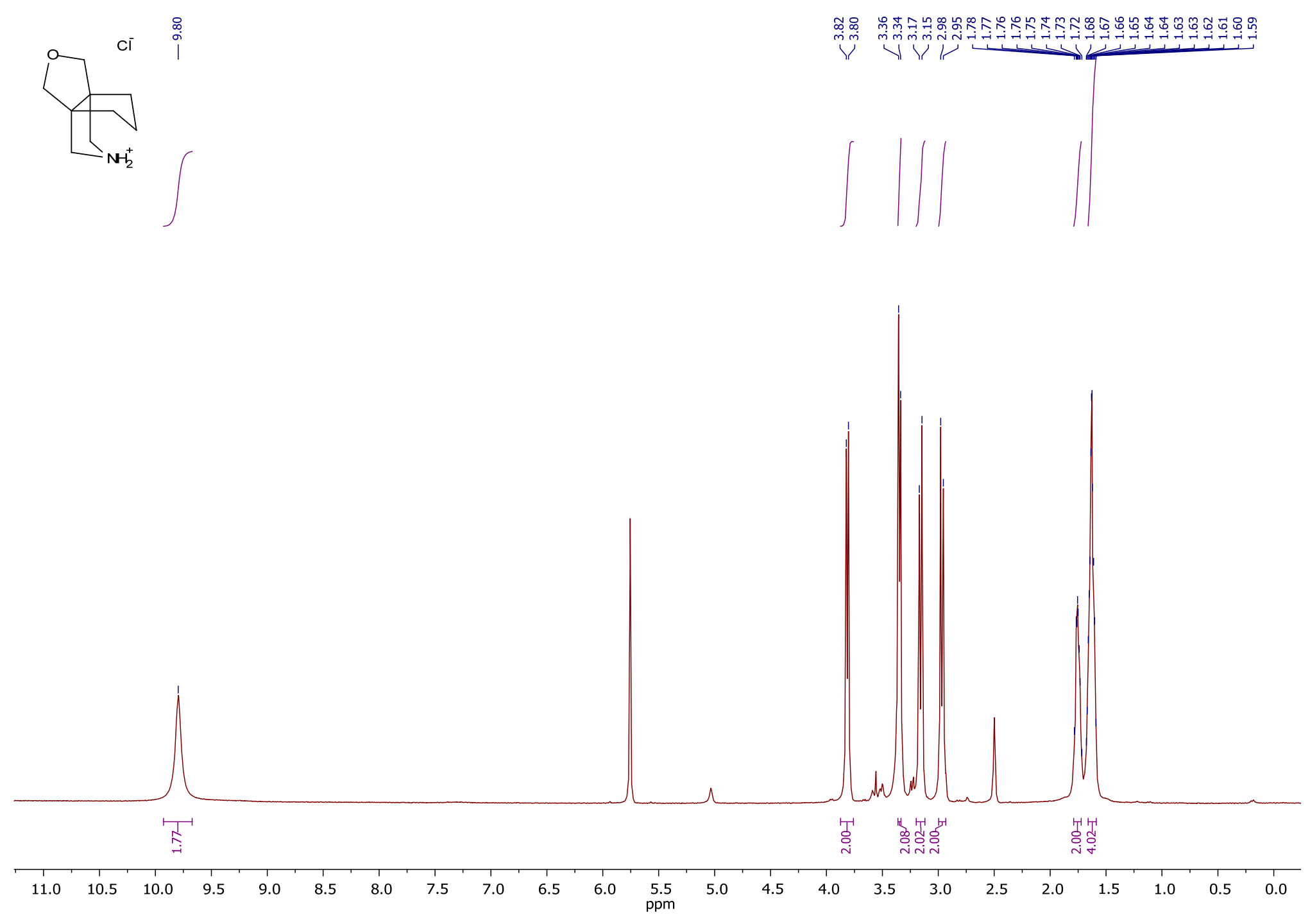

cis-Hexahydro-3a,6a-(methanooxymethano)cyclopenta[c]pyrrol-2-ium chloride (15) ${ }^{1} \mathrm{H}$ NMR 


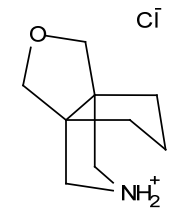

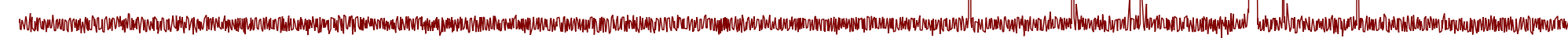

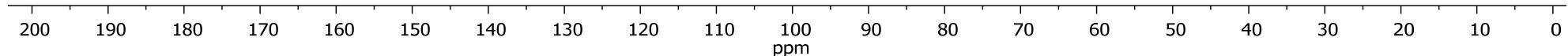

cis-Hexahydro-3a,6a-(methanooxymethano)cyclopenta[c]pyrrol-2-ium chloride $(15){ }^{13} \mathrm{C}$ NMR 


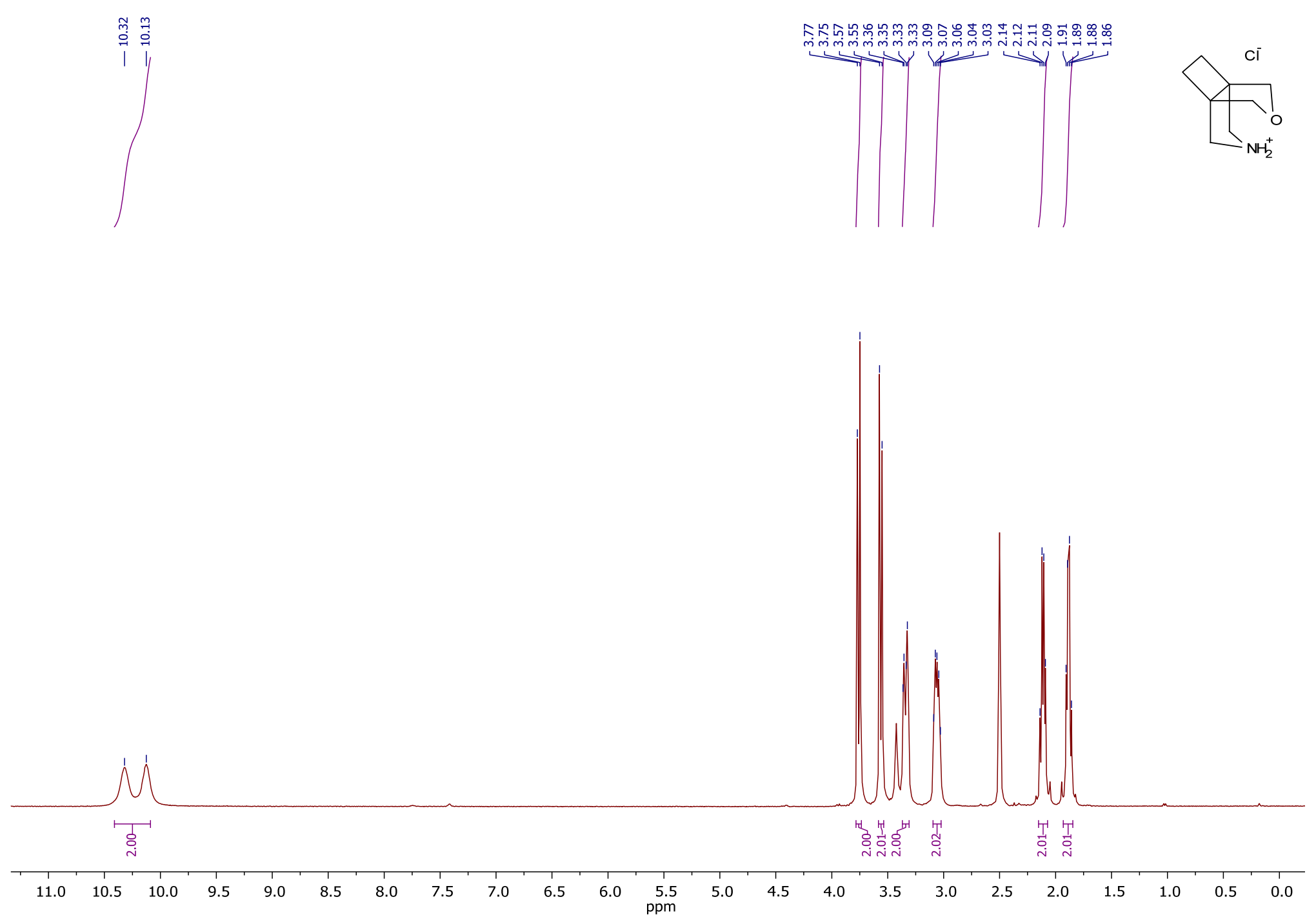

Tetrahydro-1H-3a,6a-ethanofuro[3,4-c]pyrrol-5-ium chloride (16) ${ }^{1} \mathrm{H}$ NMR 

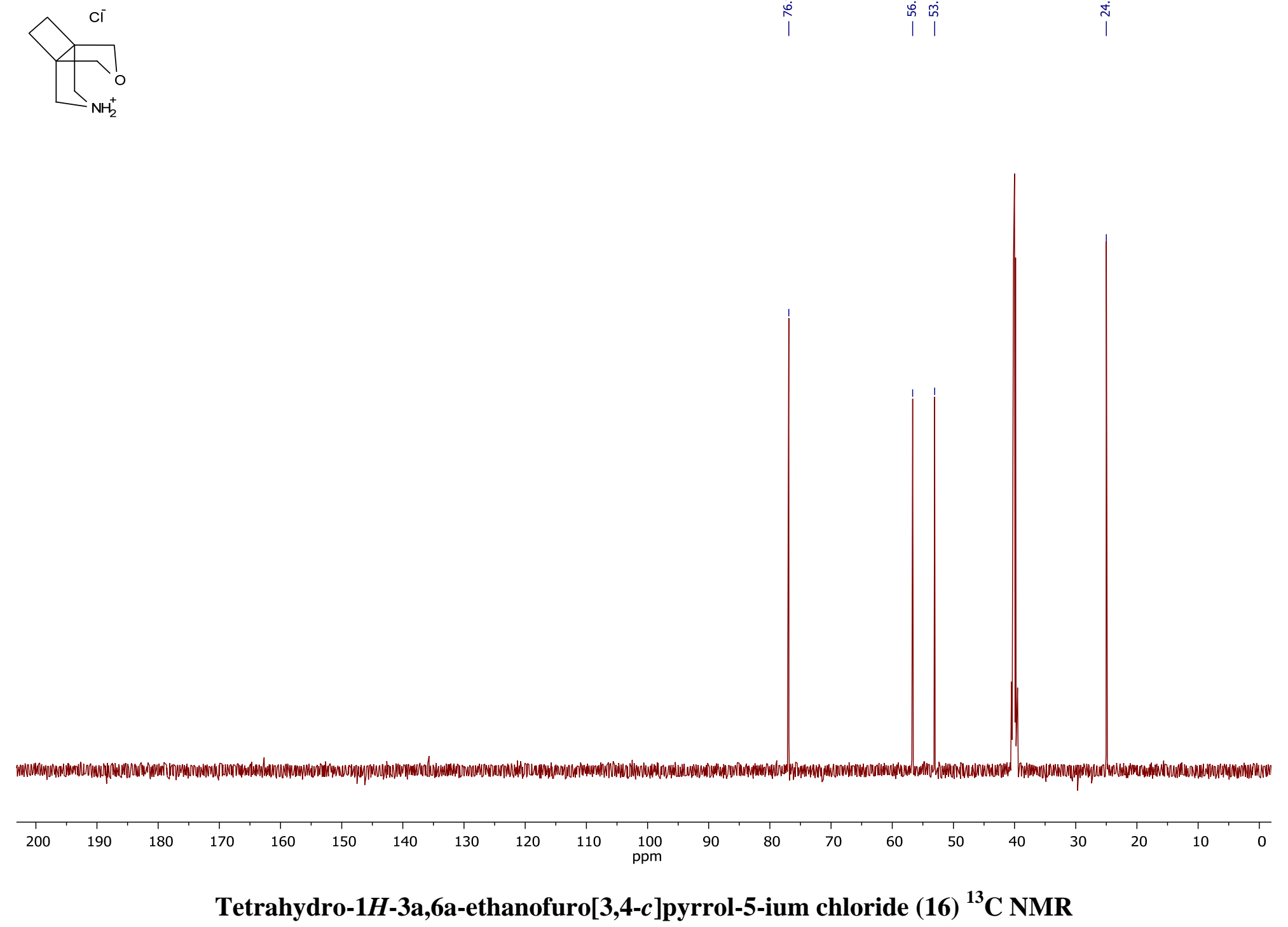


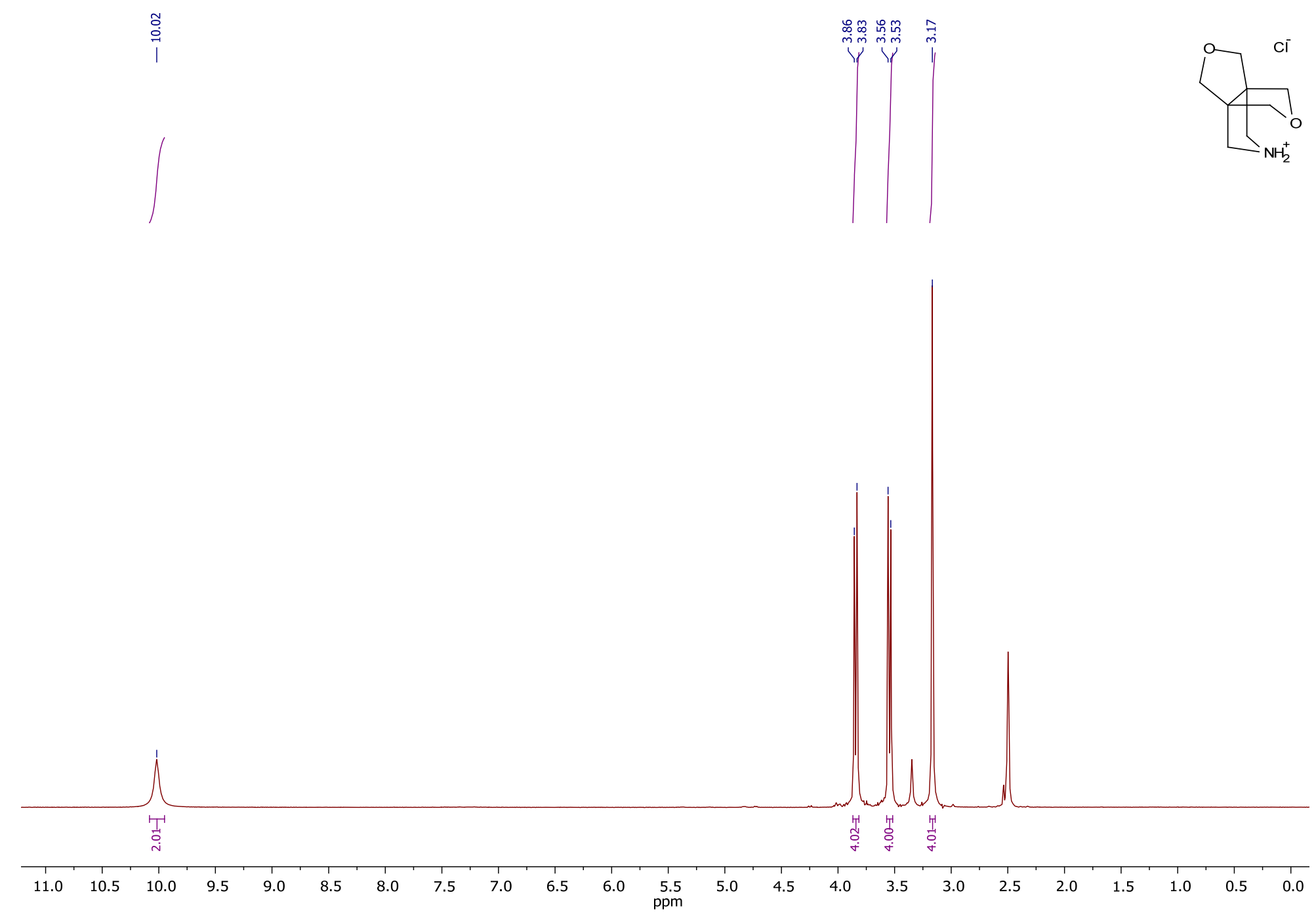

Tetrahydro-1H-3a,6a-(methanooxymethano)furo[3,4-c]pyrrol-5-ium chloride (17) ${ }^{1} \mathrm{H}$ NMR 

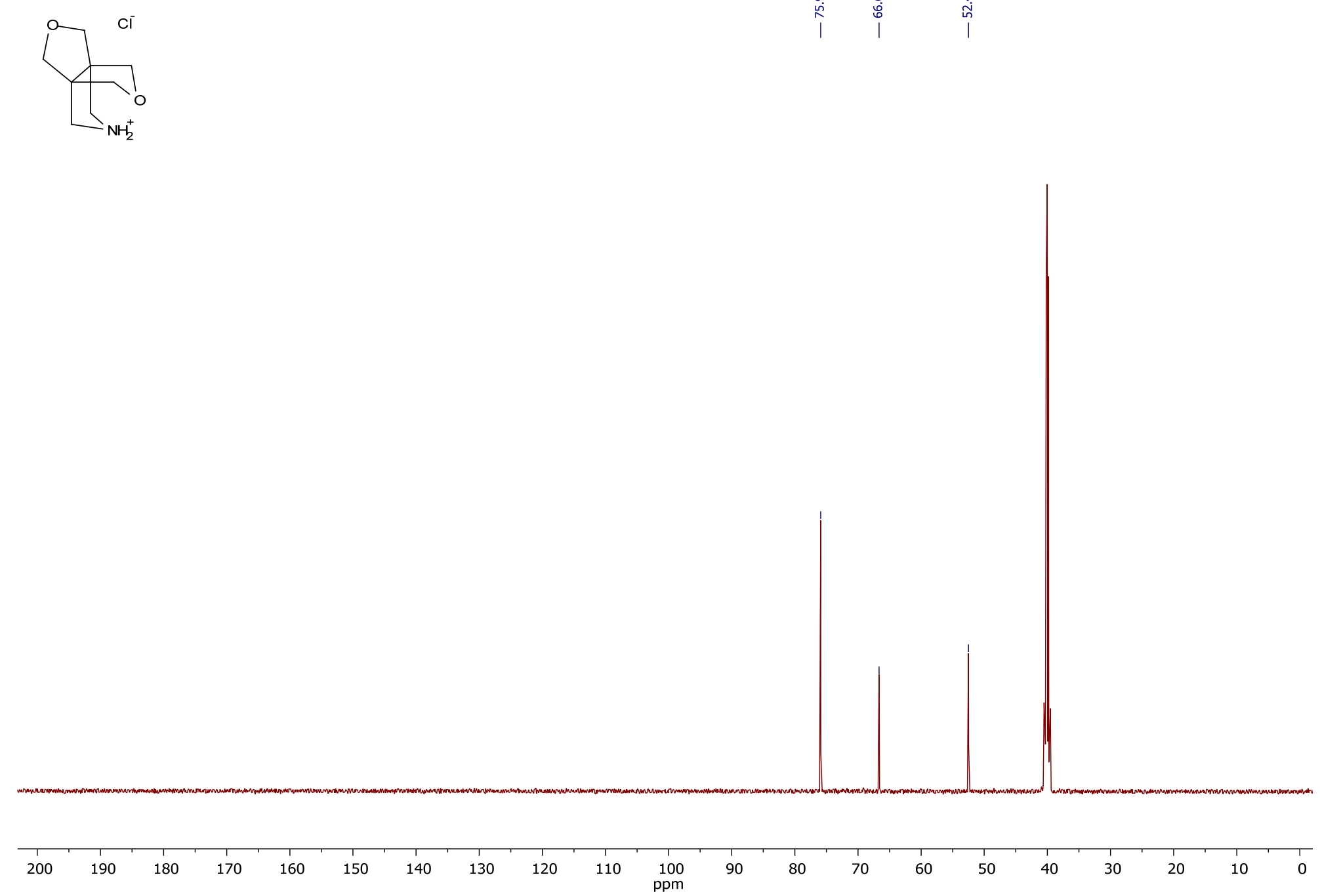

Tetrahydro-1H-3a,6a-(methanooxymethano)furo[3,4-c]pyrrol-5-ium chloride (17) ${ }^{13} \mathrm{C}$ NMR 


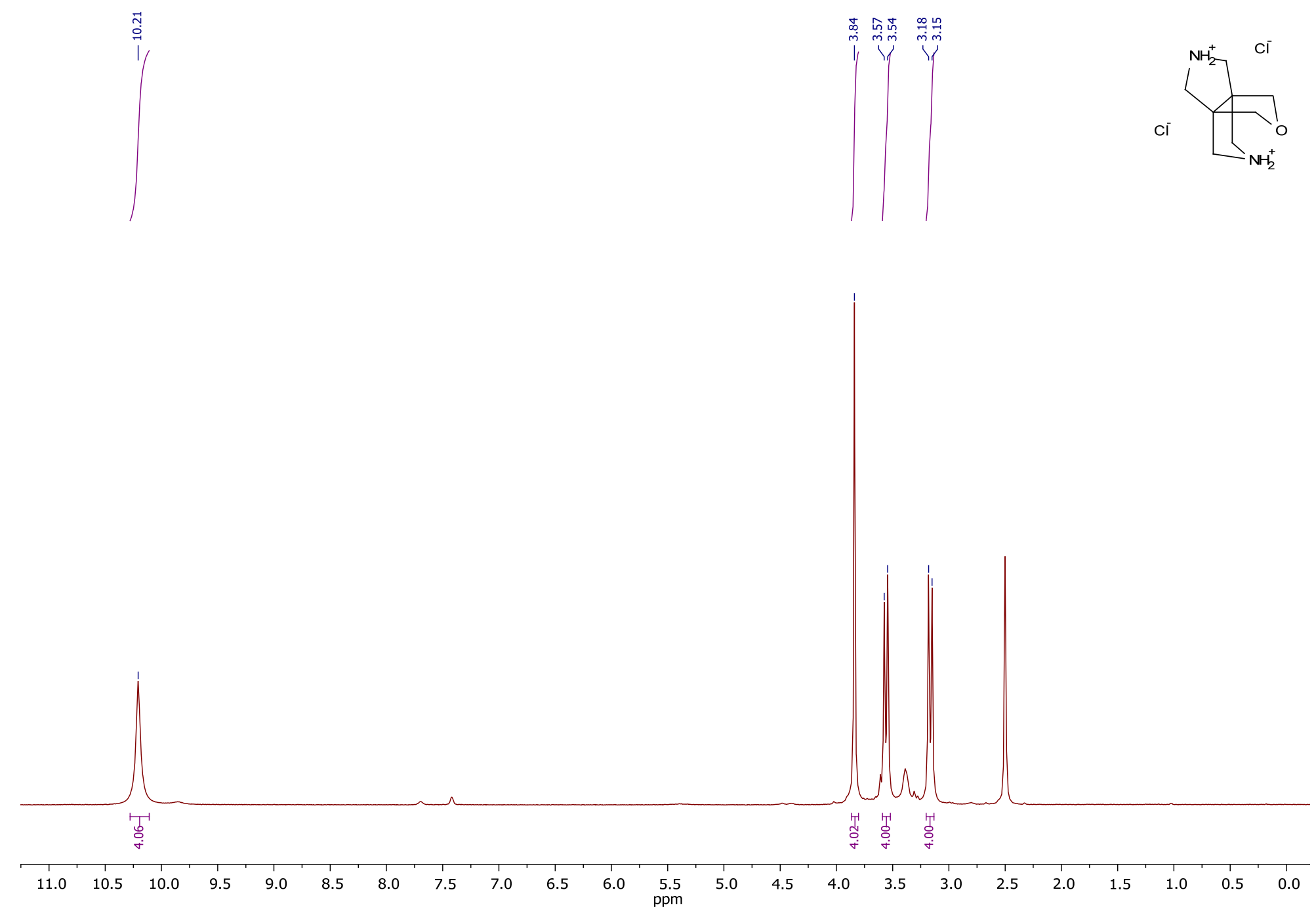

Hexahydro-3a,6a-(methanooxymethano)pyrrolo[3,4-c]pyrrole-2,5-diium chloride (18) ${ }^{1} \mathrm{H}$ NMR 
Cī
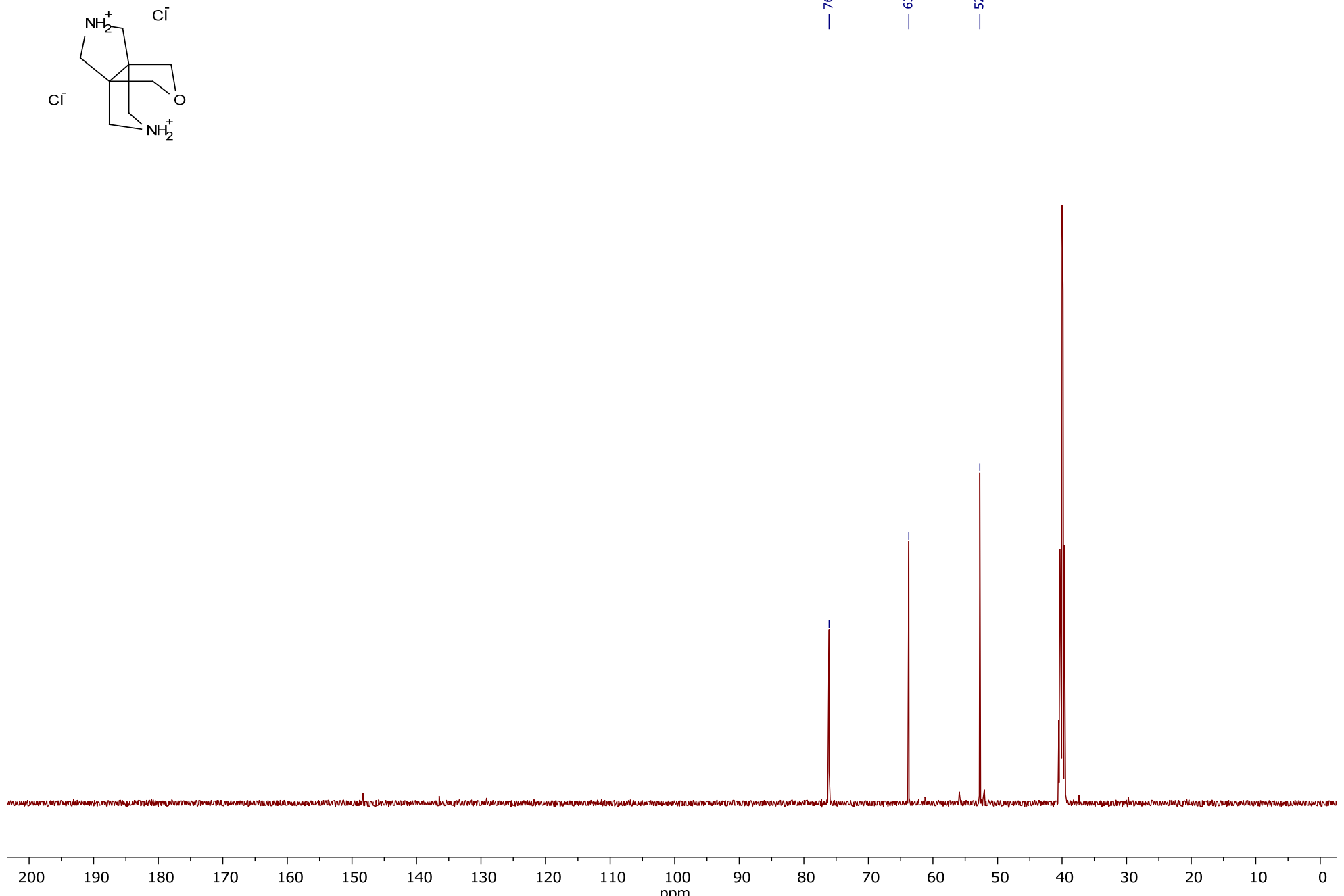

Hexahydro-3a,6a-(methanooxymethano)pyrrolo[3,4-c]pyrrole-2,5-diium chloride (18) ${ }^{13} \mathrm{C}$ NMR 
Cī
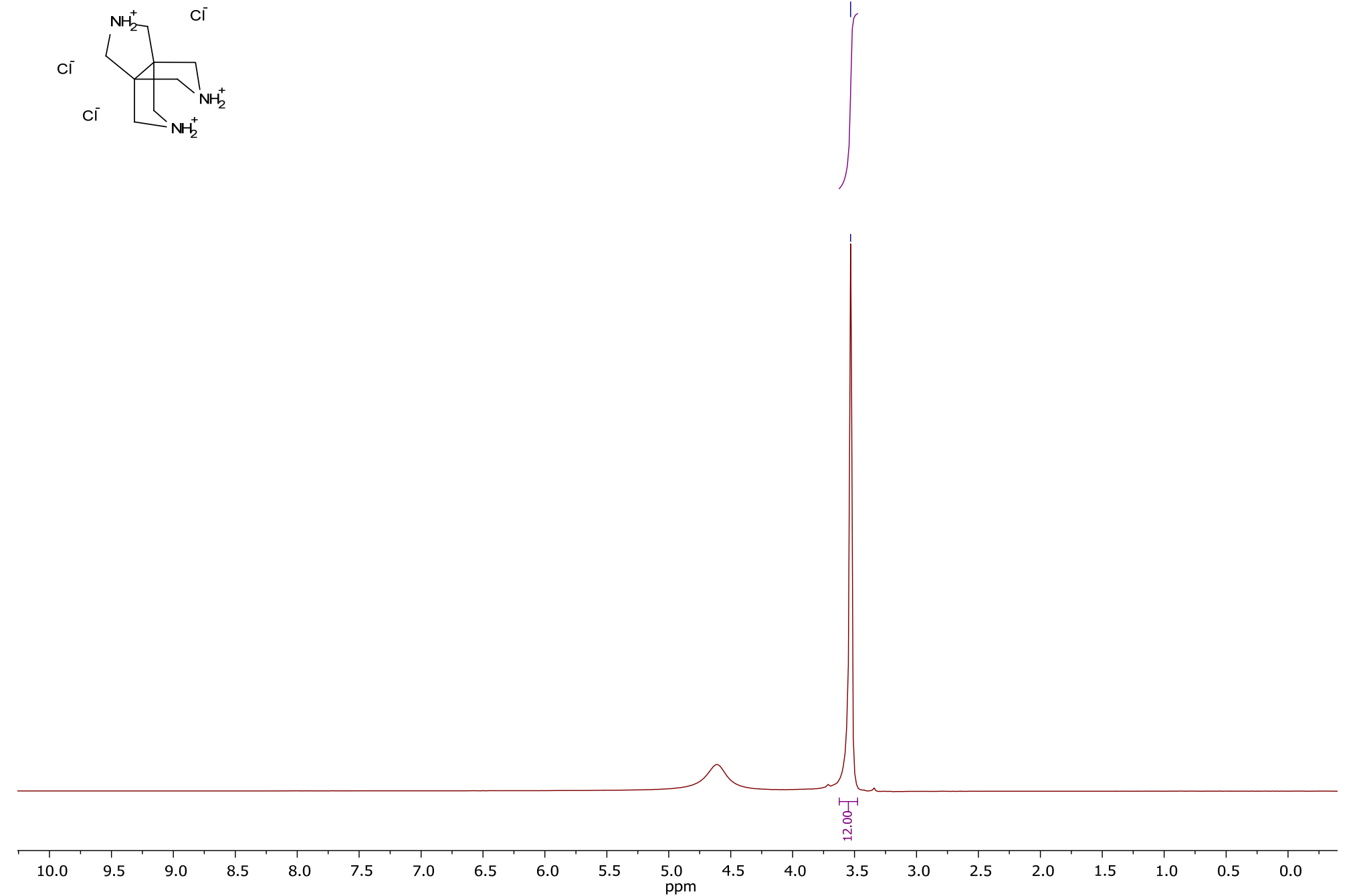

Hexahydro-3a,6a-(methanoiminomethano)pyrrolo[3,4-c]pyrrole-2,5-diium chloride (19) ${ }^{1} \mathrm{H}$ NMR 

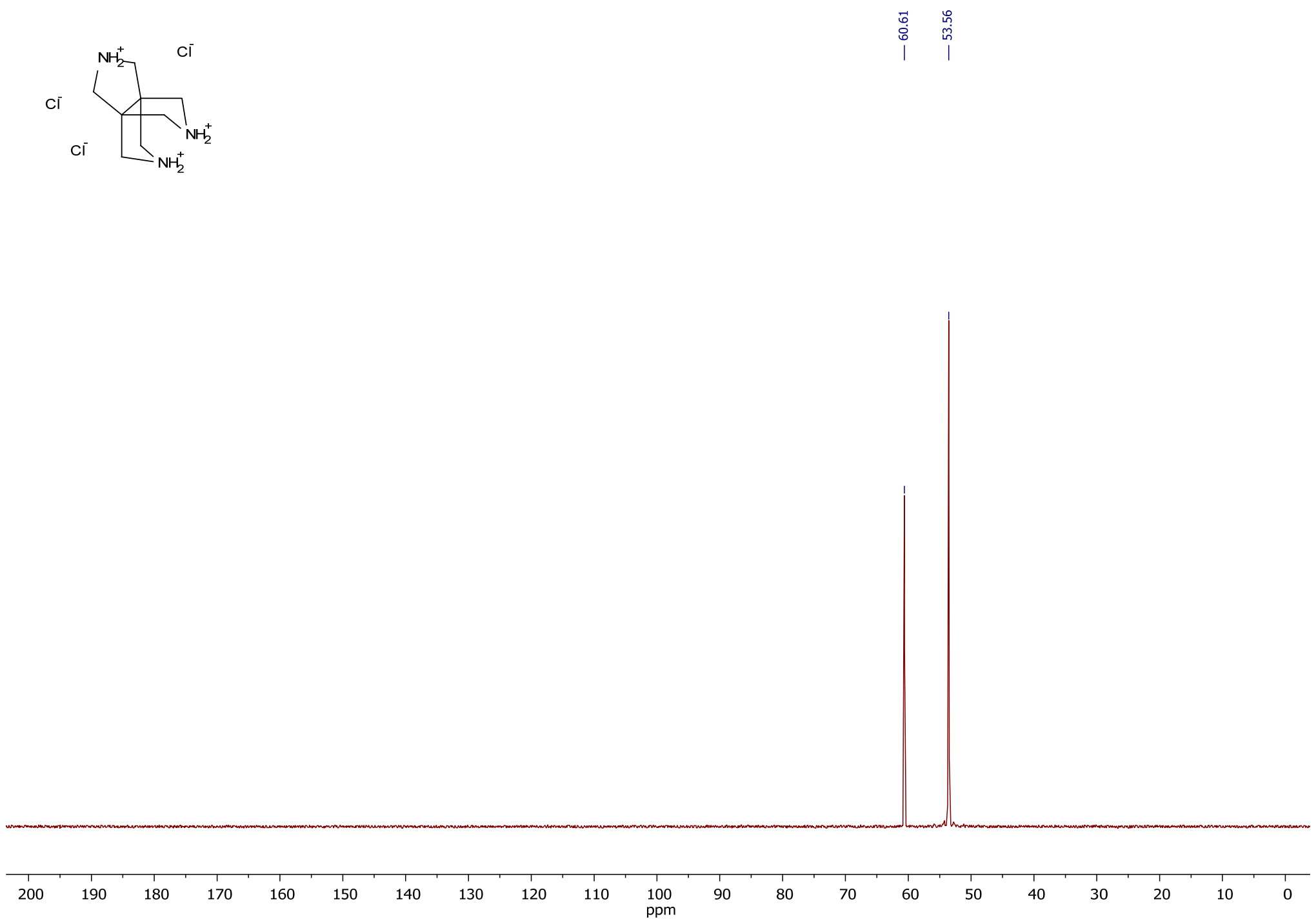

Hexahydro-3a,6a-(methanoiminomethano)pyrrolo[3,4-c]pyrrole-2,5-diium chloride (19) ${ }^{13} \mathrm{C}$ NMR 

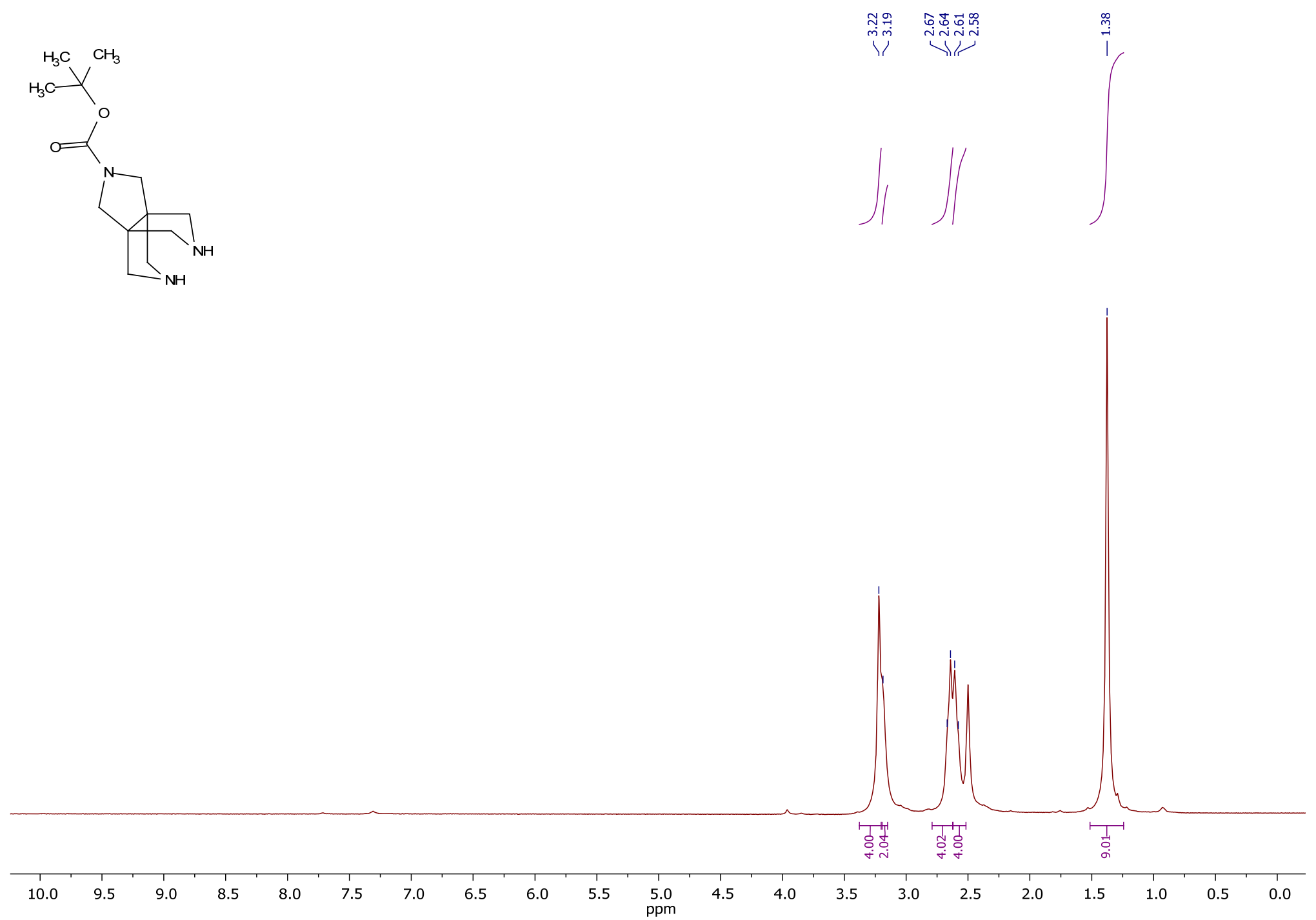

tert-butyl tetrahydro-3a,6a-(methanoiminomethano)pyrrolo[3,4-c]pyrrole-2(1H)-carboxylate (20) ${ }^{1}$ H NMR 


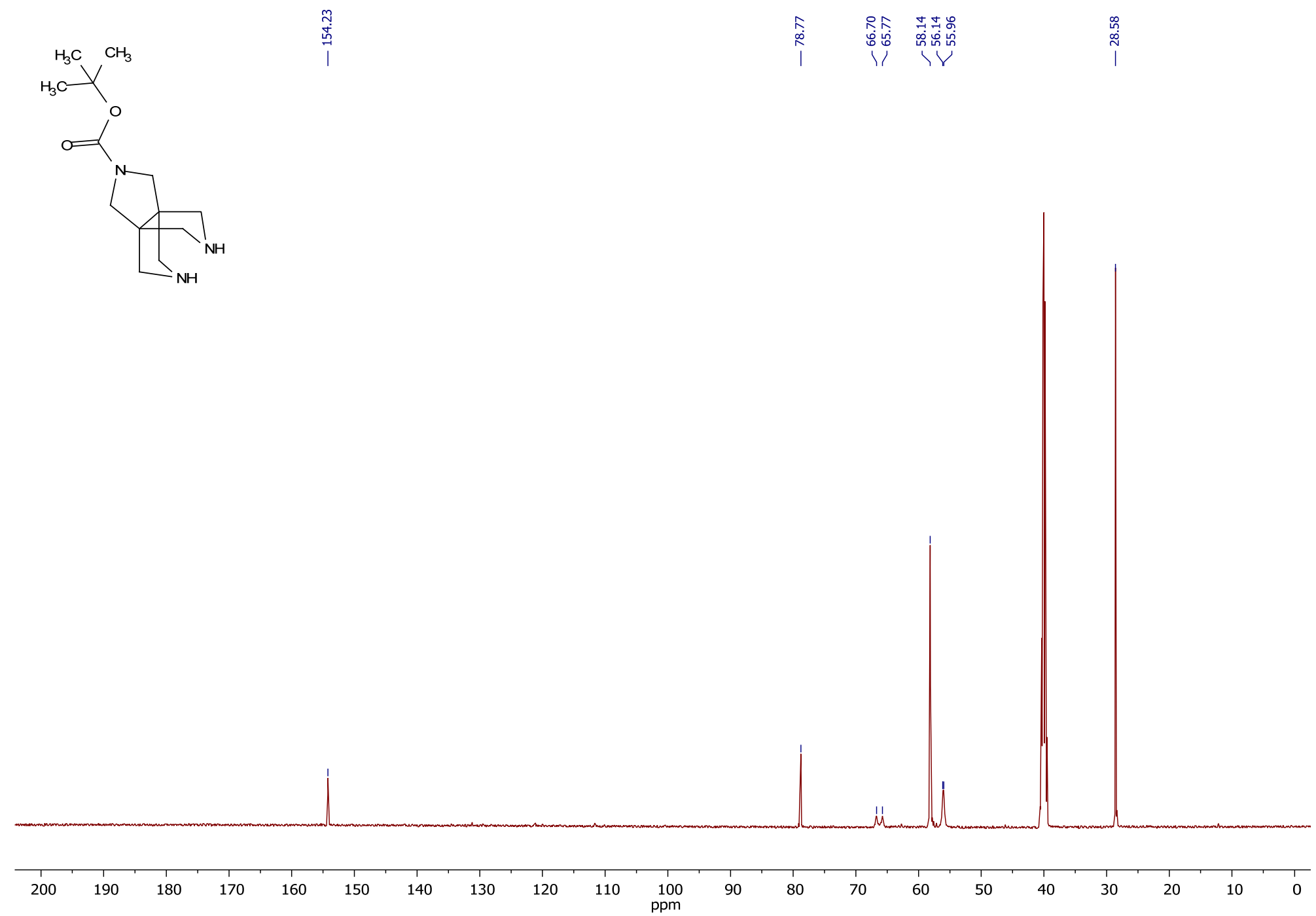

tert-butyl tetrahydro-3a,6a-(methanoiminomethano)pyrrolo[3,4-c]pyrrole-2(1H)-carboxylate (20) ${ }^{1}$ H NMR 


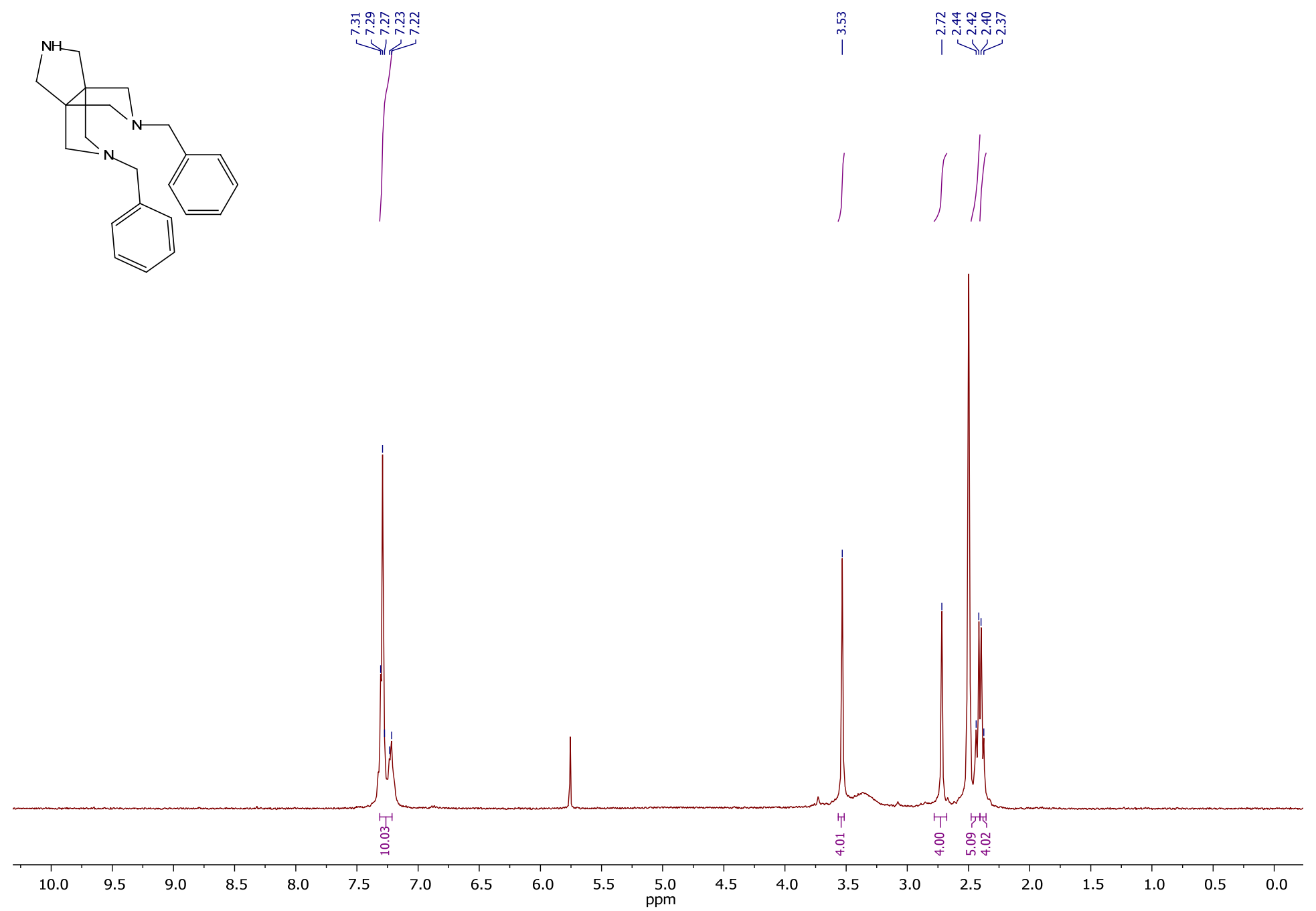

2,5-Dibenzylhexahydro-3a,6a-(methanoiminomethano)pyrrolo[3,4-c]pyrrole (21) ${ }^{1} \mathrm{H}$ NMR 


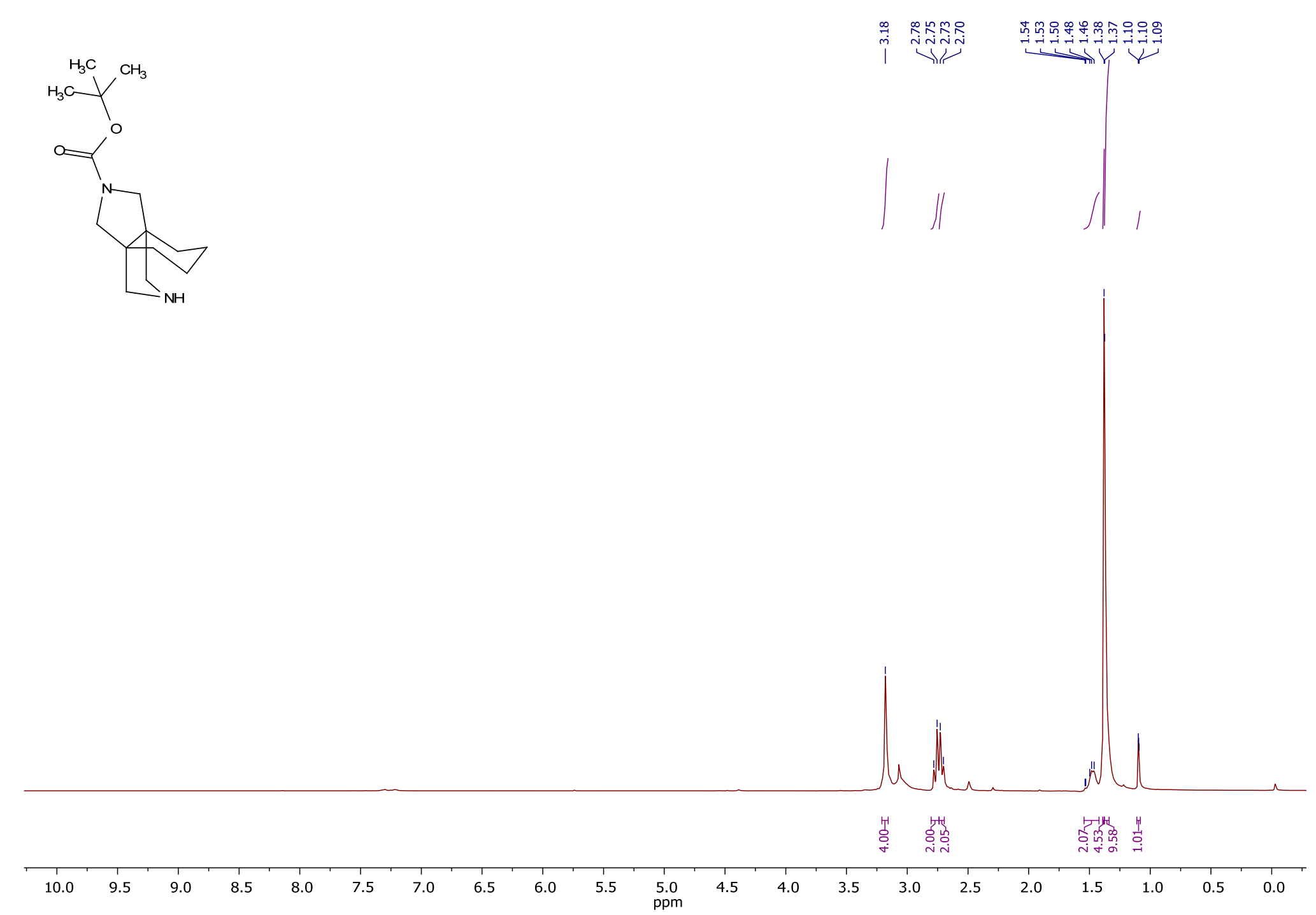

tert-Butyl tetrahydro-1H-3a,7a-(methanoiminomethano)isoindole-2(3H)-carboxylate (22) ${ }^{1} \mathrm{H}$ NMR 


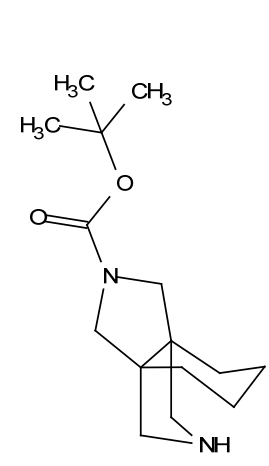

|

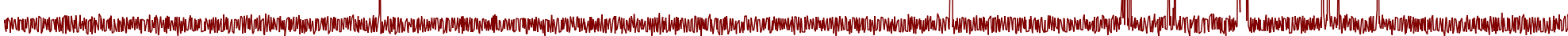

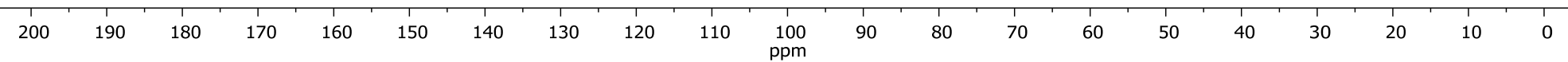

tert-Butyl tetrahydro-1H-3a,7a-(methanoiminomethano)isoindole-2(3H)-carboxylate $(22){ }^{13} \mathrm{C}$ NMR 


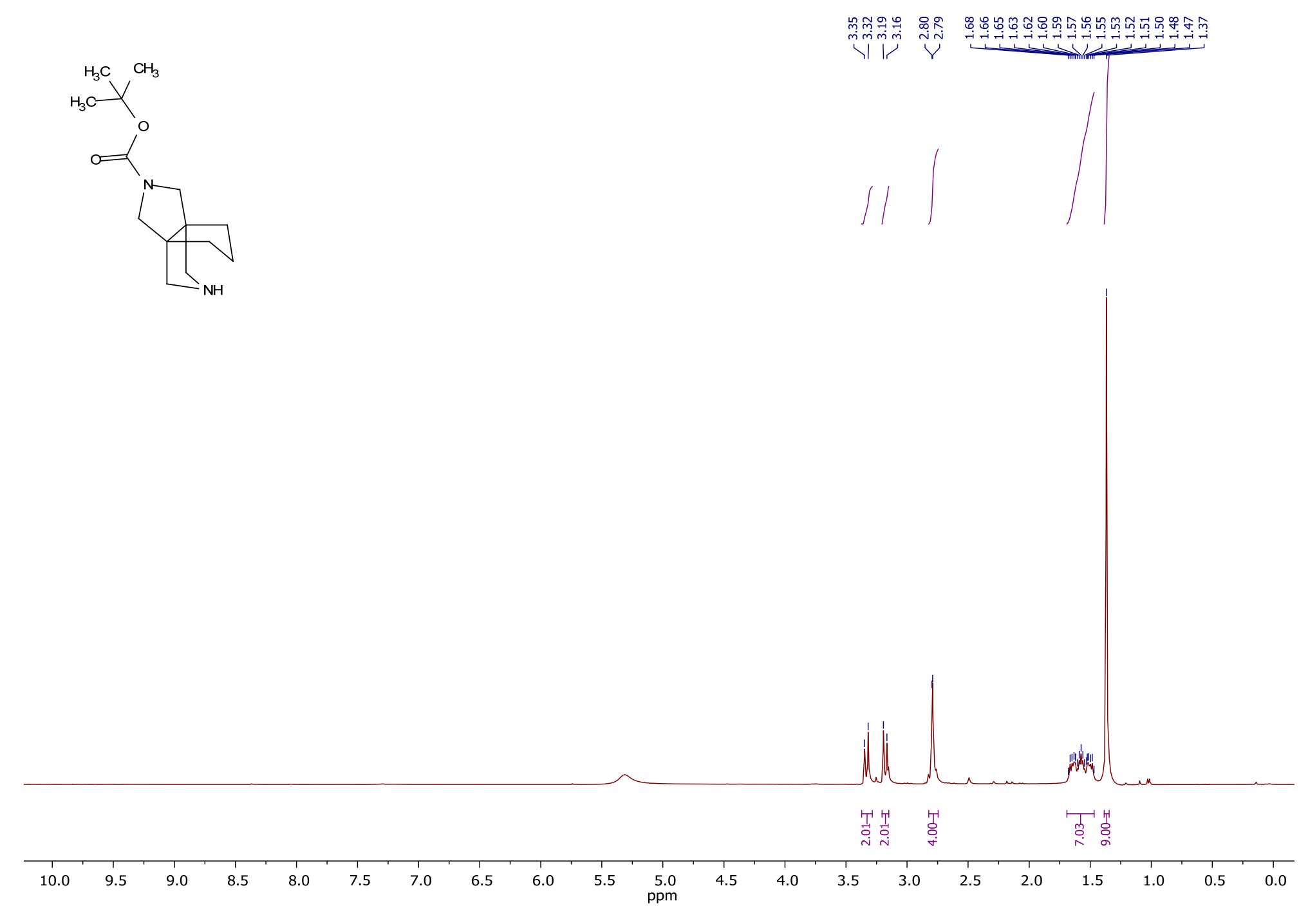

tert-Butyl tetrahydro-3a,6a-(methanoiminomethano)cyclopenta[c]pyrrole-2(1H)-carboxylate (23) ${ }^{1} \mathrm{H}$ NMR 


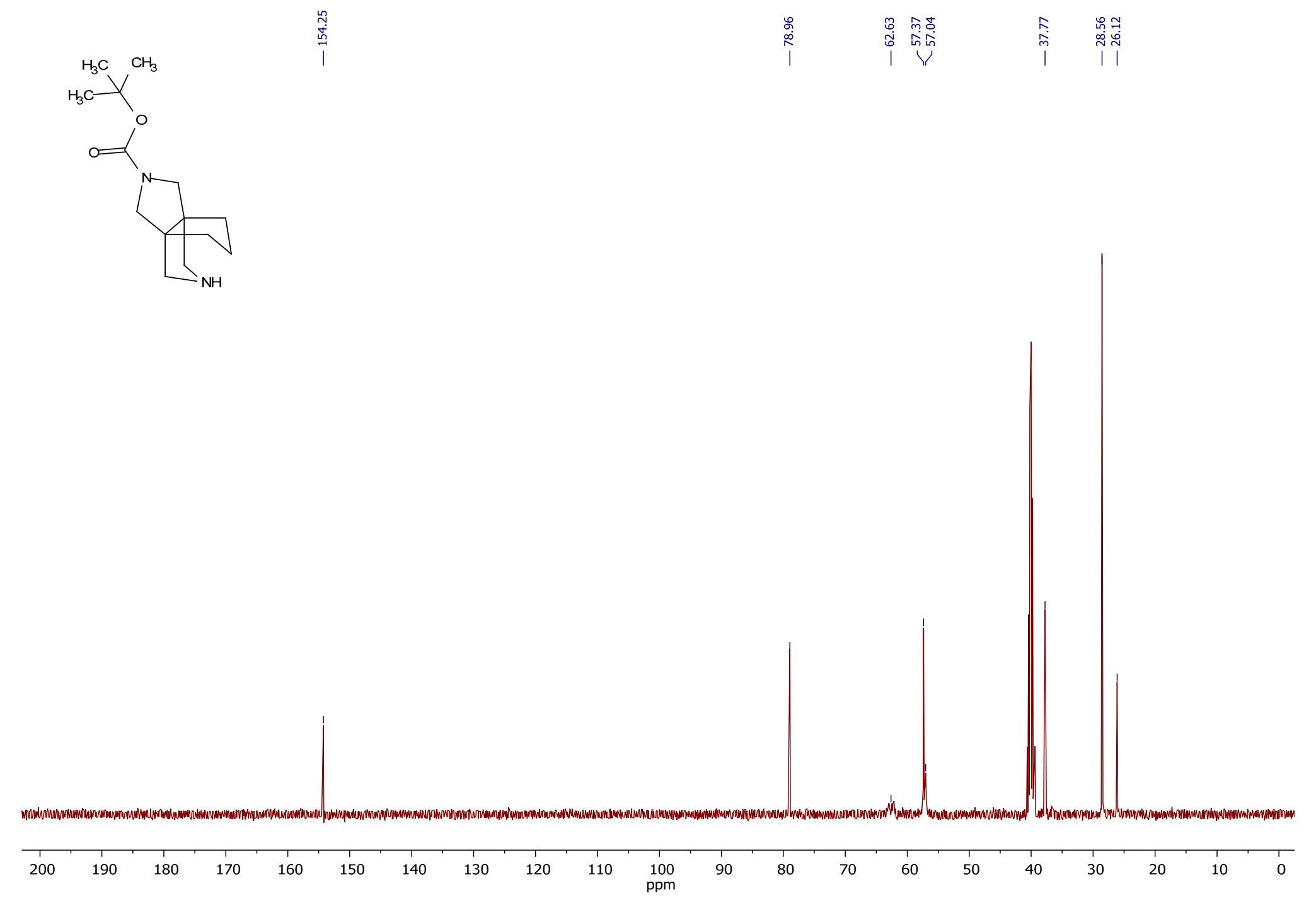

tert-Butyl tetrahydro-3a,6a-(methanoiminomethano)cyclopenta[c]pyrrole-2(1H)-carboxylate (23) ${ }^{13} \mathrm{C}$ NMR 

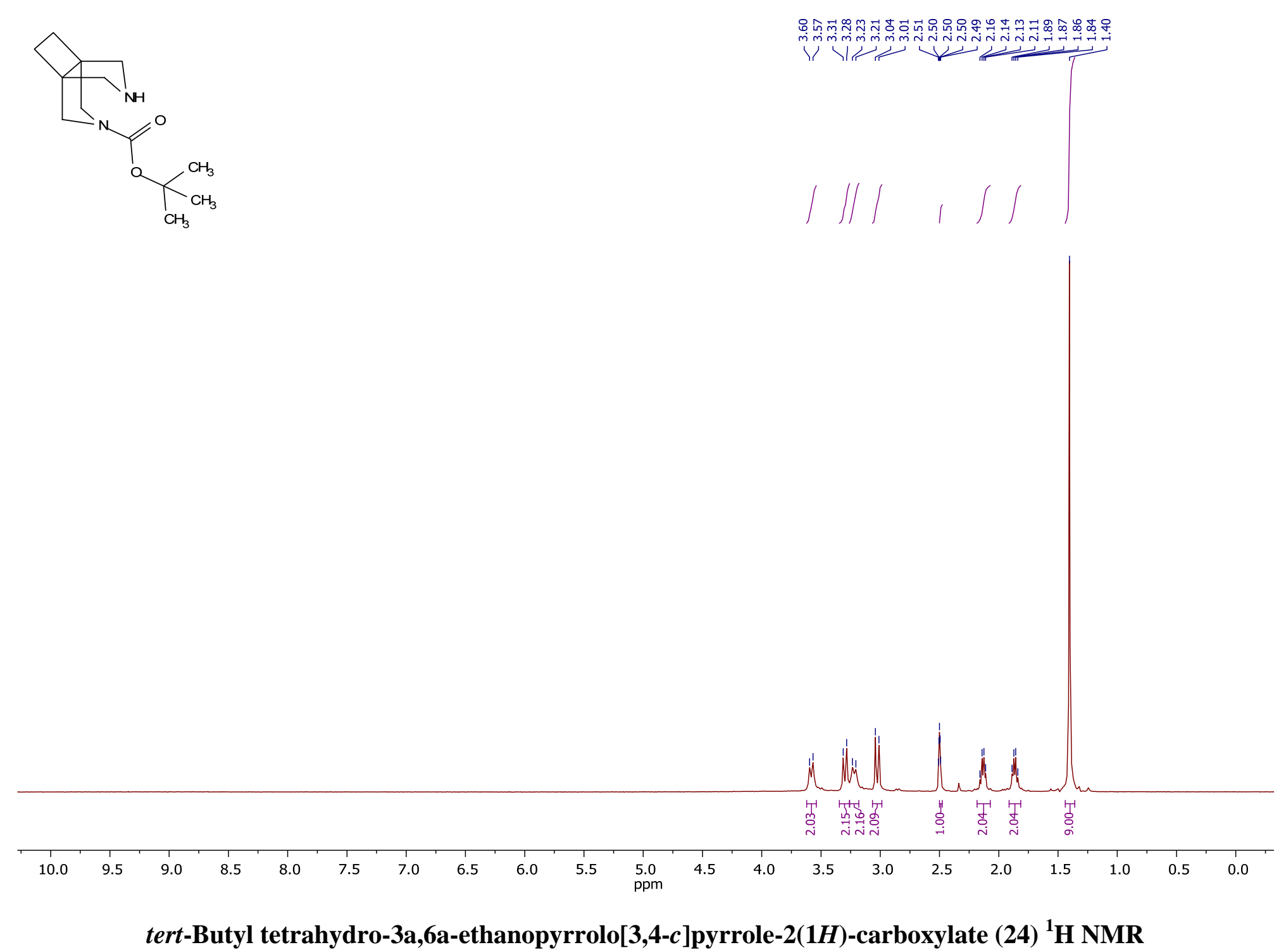


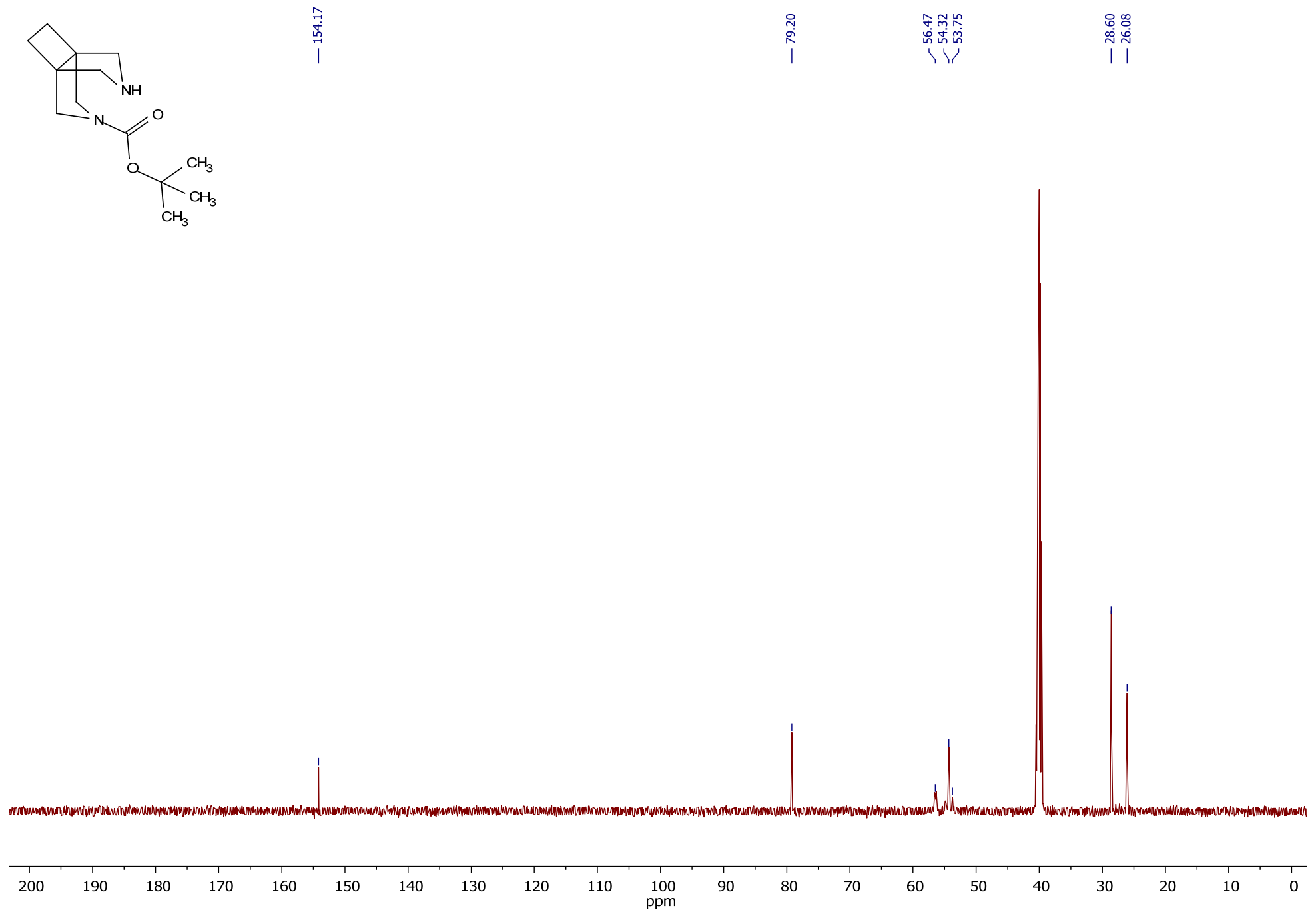

tert-Butyl tetrahydro-3a,6a-ethanopyrrolo[3,4-c]pyrrole-2(1H)-carboxylate (24) ${ }^{13} \mathrm{C}$ NMR 


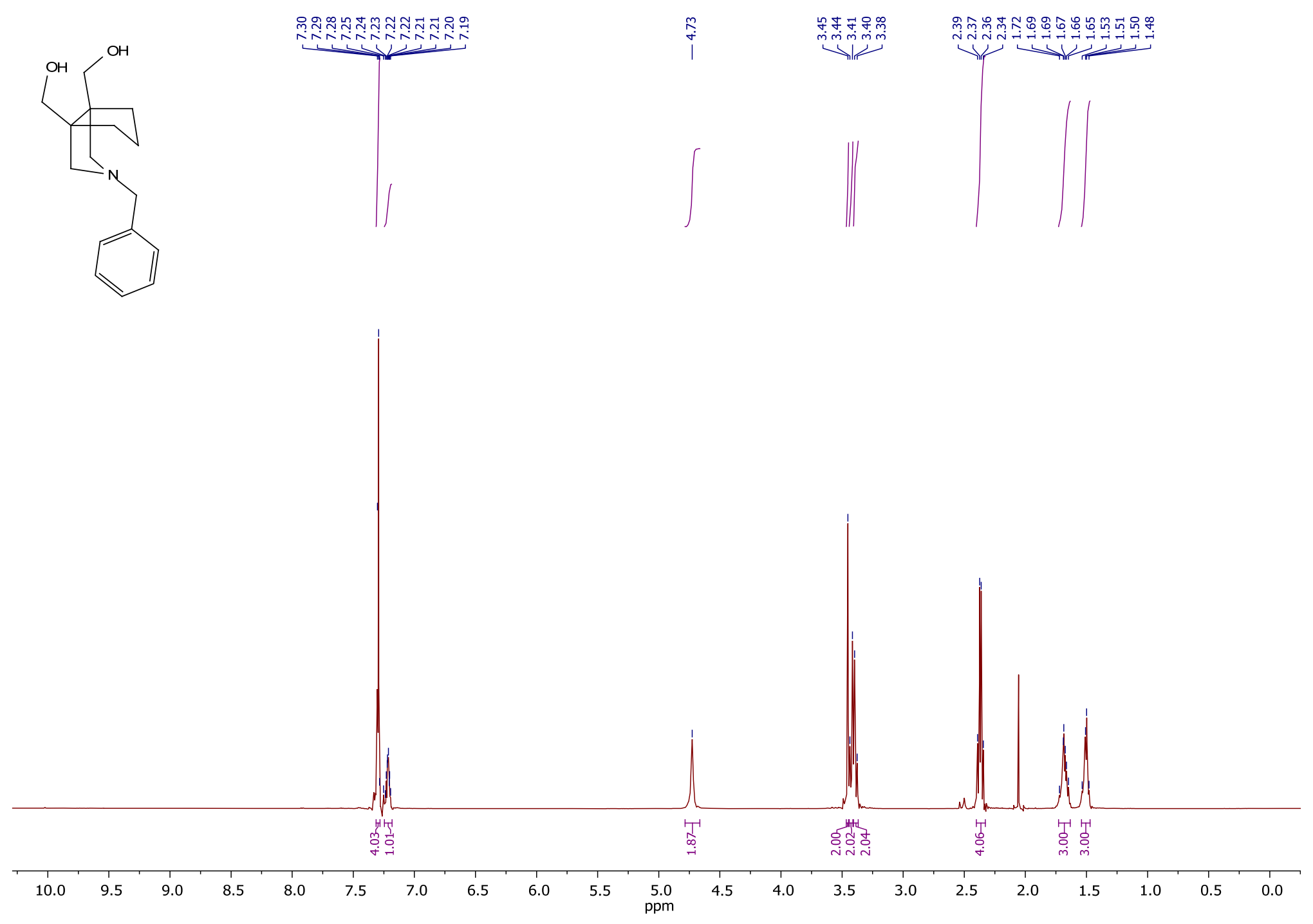

cis-2-Benzyloctahydrocyclopenta[c]pyrrole-3a,6a-diyl)dimethanol (32) ${ }^{1}$ H NMR 


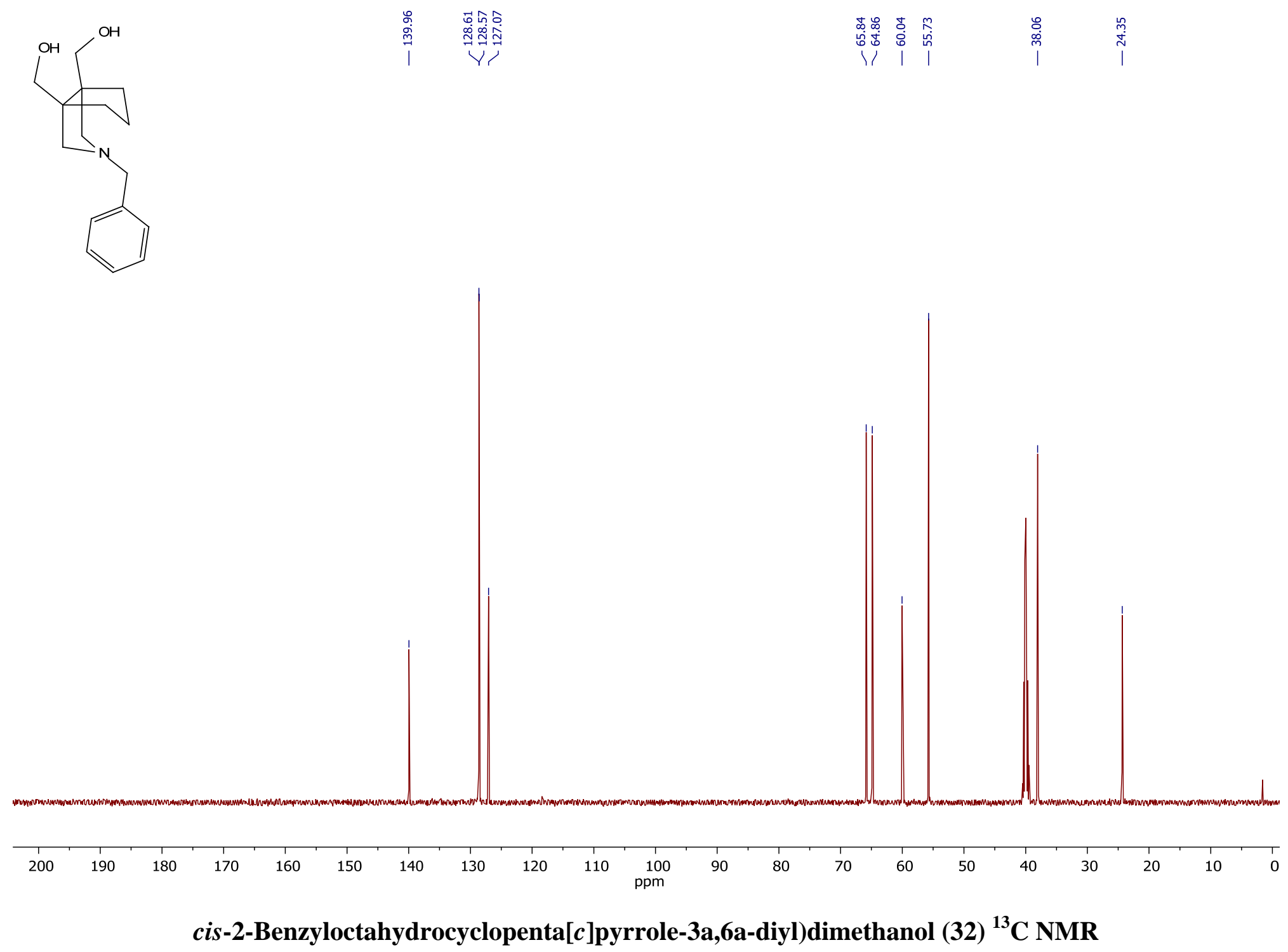




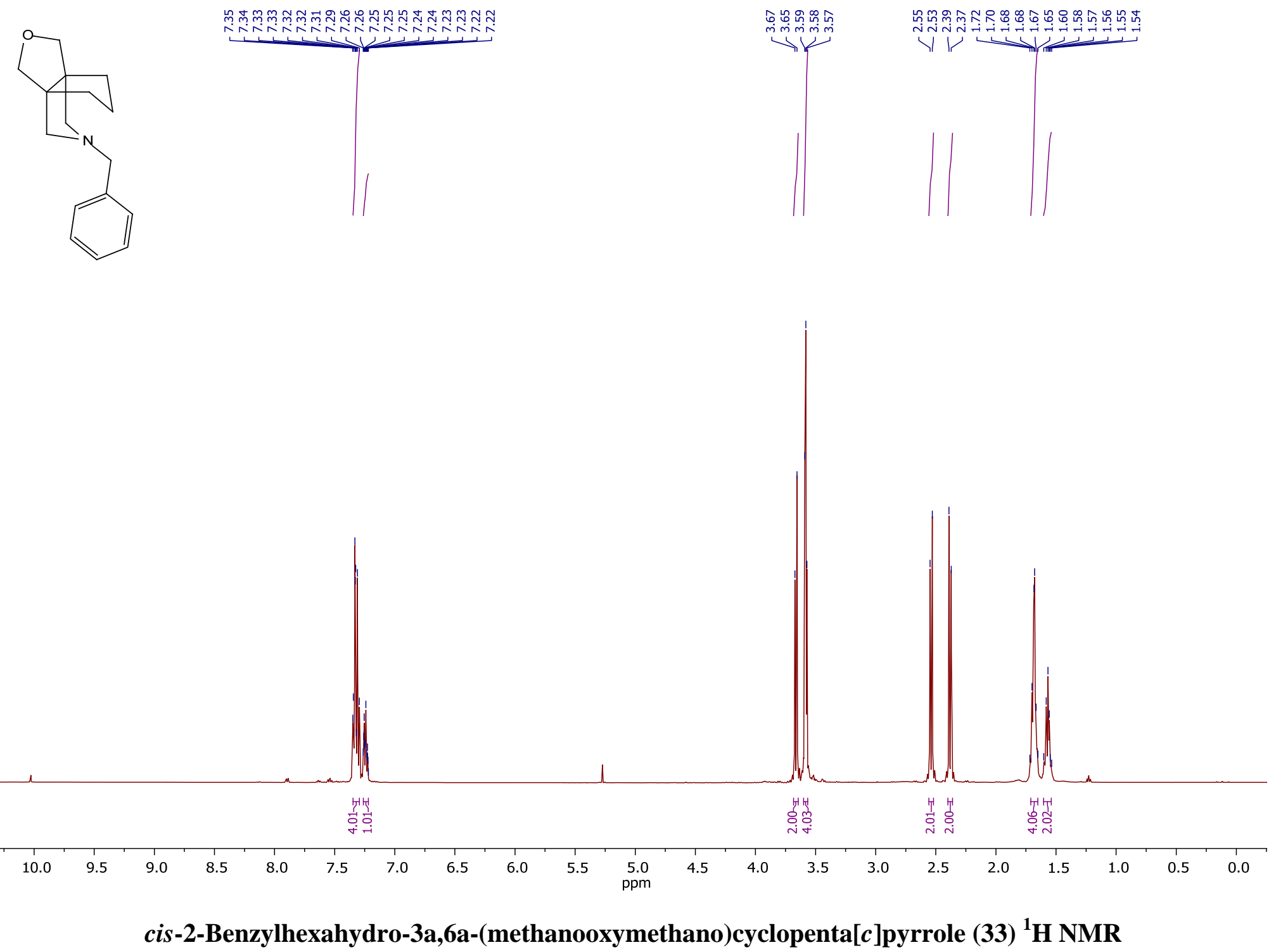




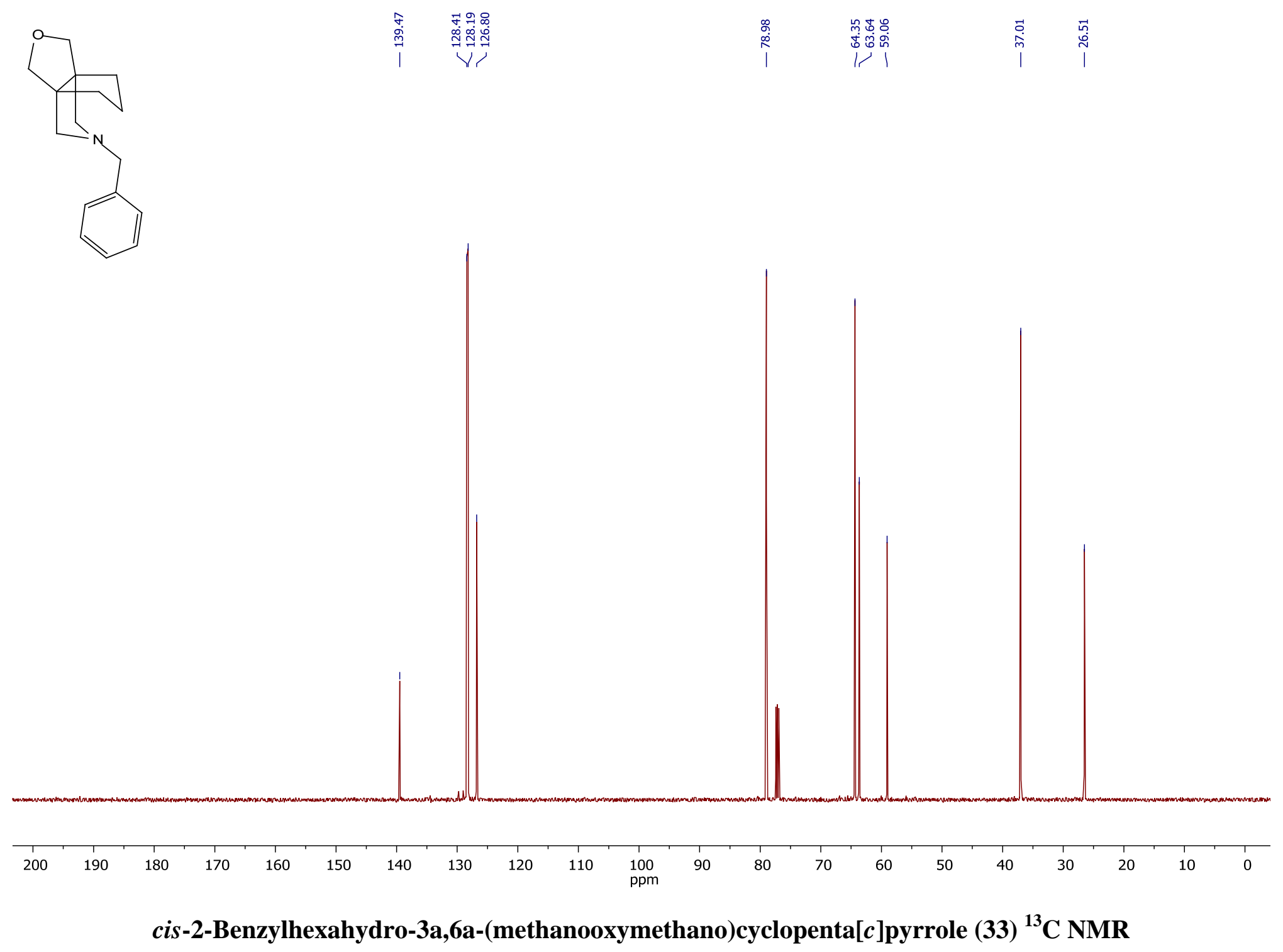




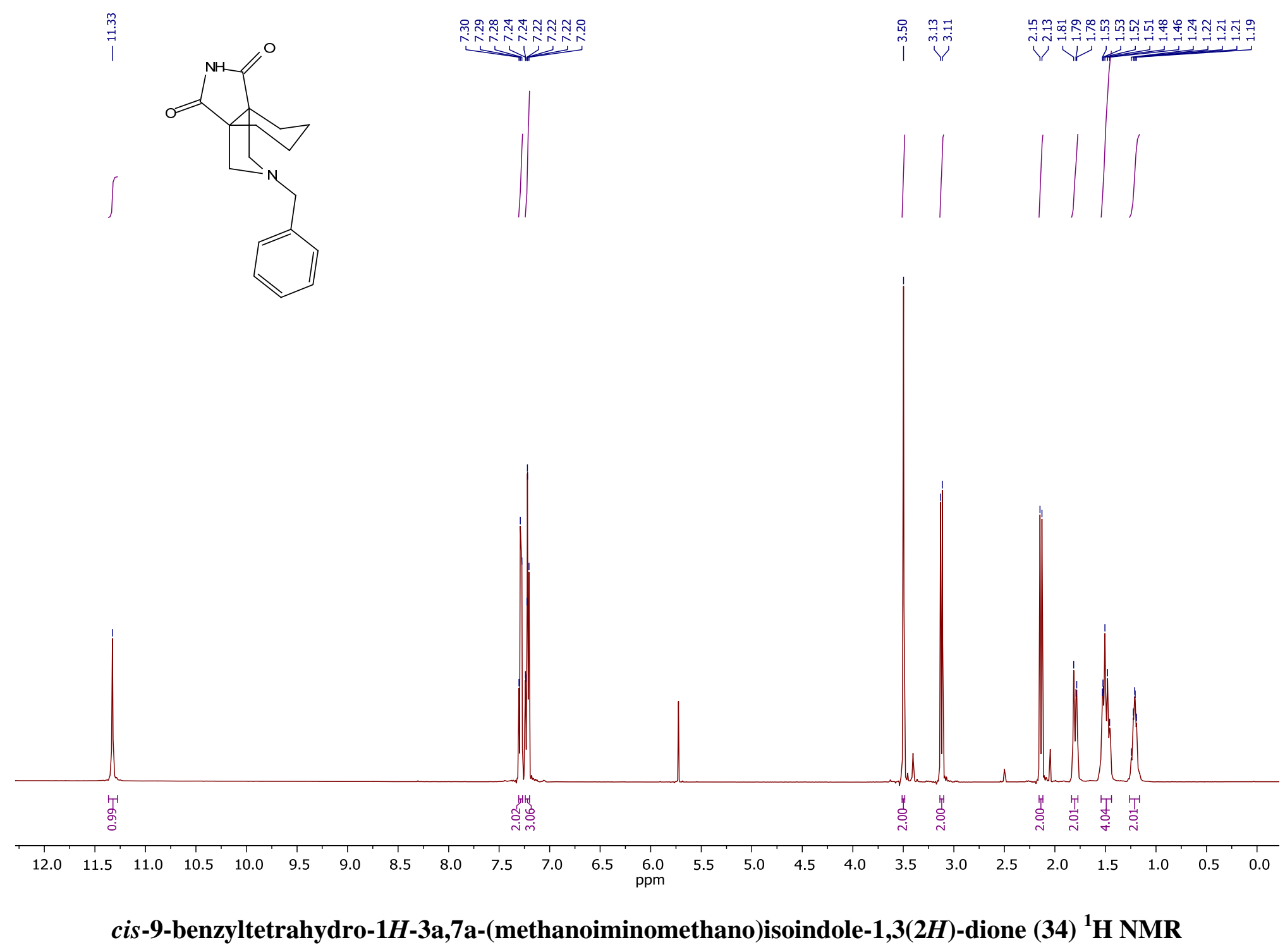




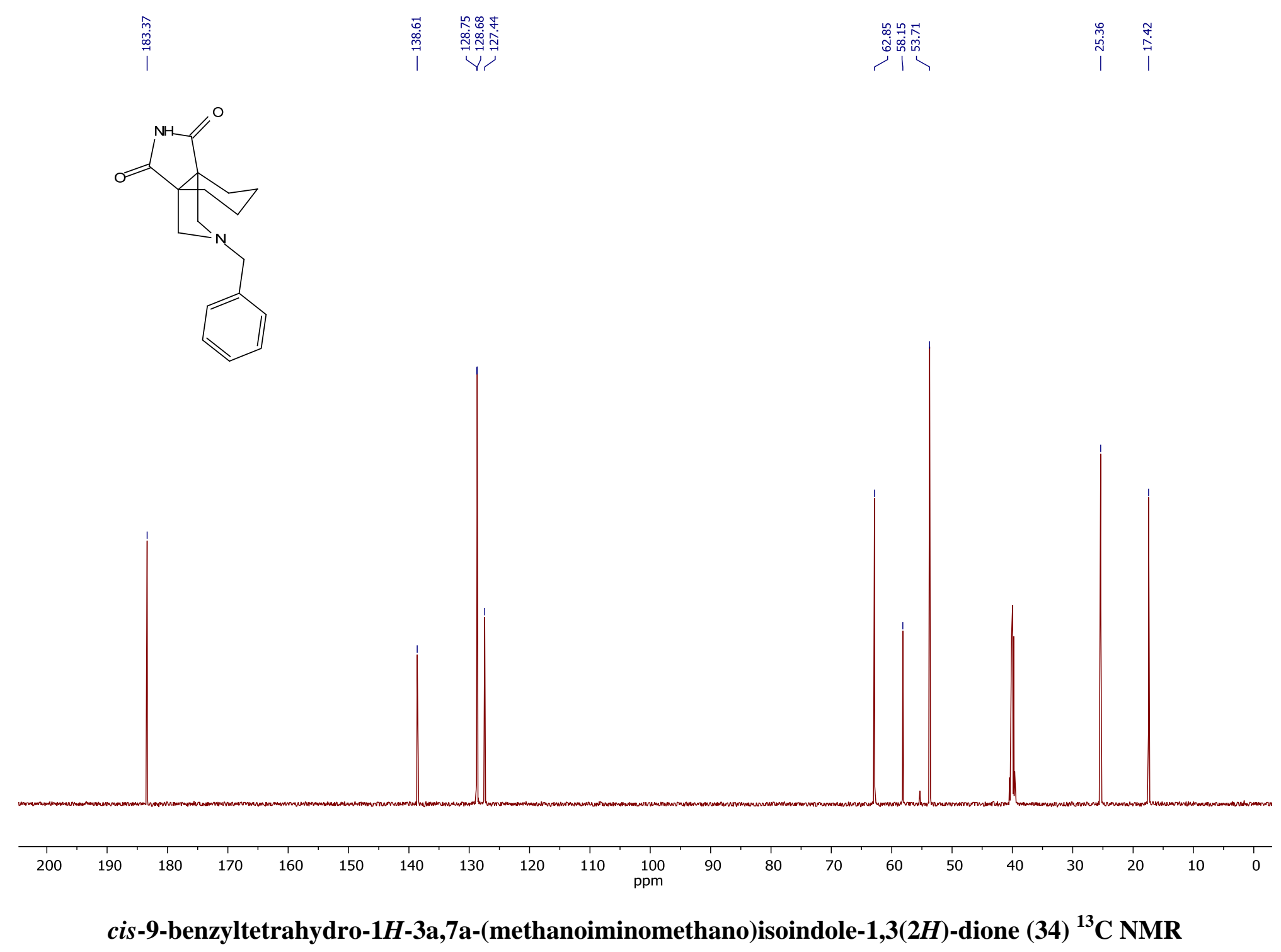




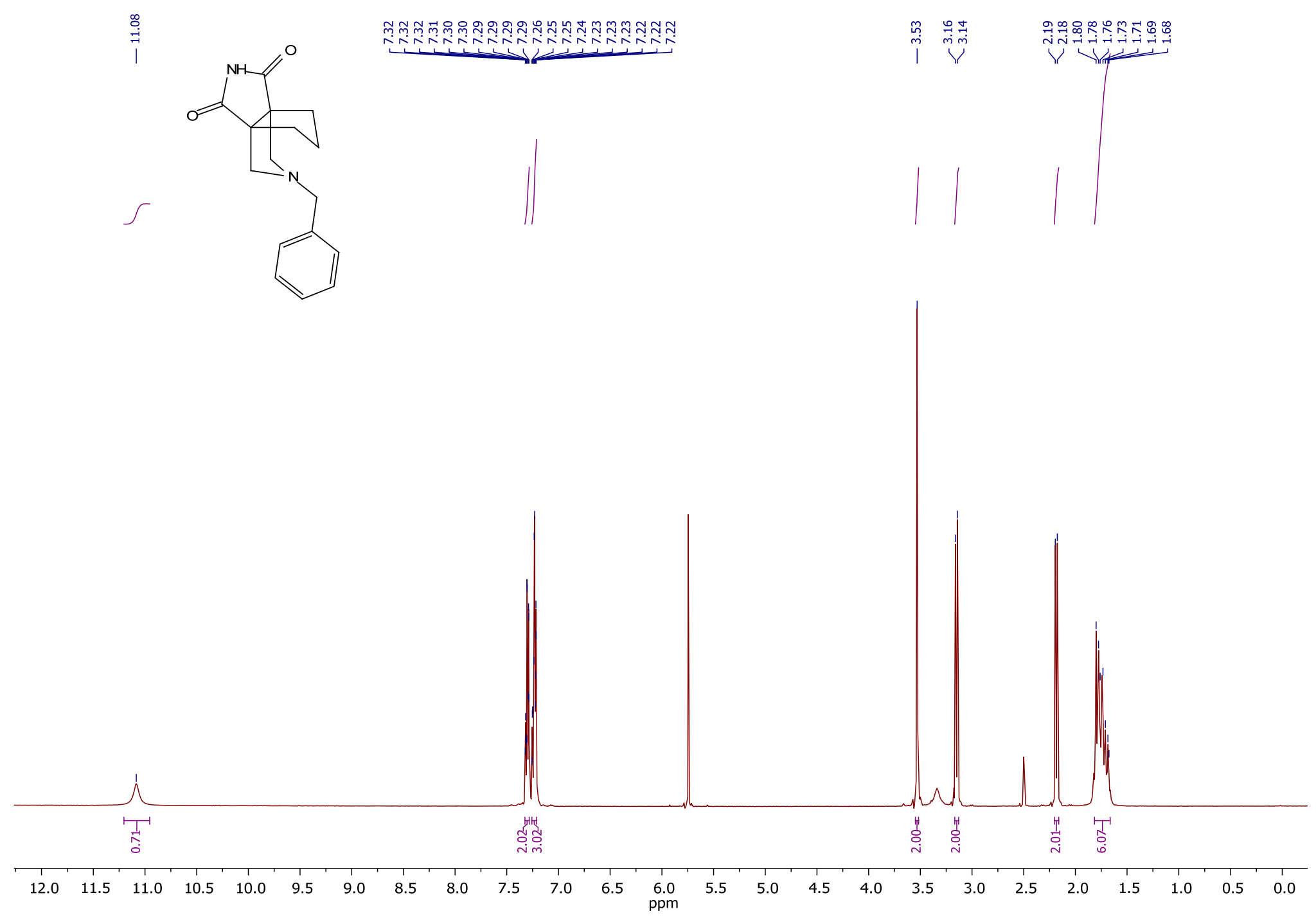

cis-8-Benzyldihydro-3a,6a-(methanoiminomethano)cyclopenta[c]pyrrole-1,3(2H,4H)-dione (35) ${ }^{1} \mathrm{H}$ NMR 


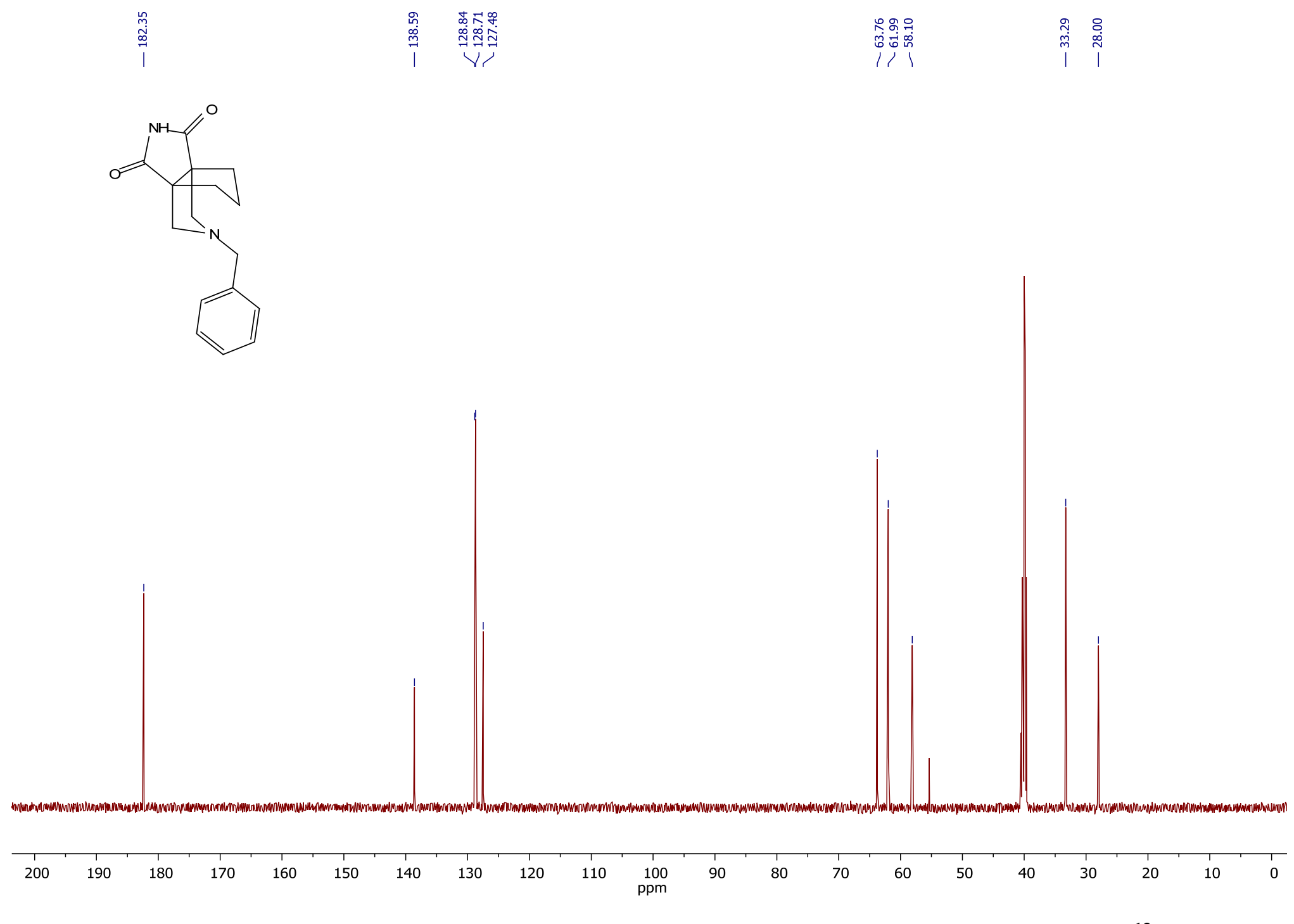

cis-8-Benzyldihydro-3a,6a-(methanoiminomethano)cyclopenta[c]pyrrole-1,3(2H,4H)-dione (35) ${ }^{13} \mathrm{C}$ NMR 


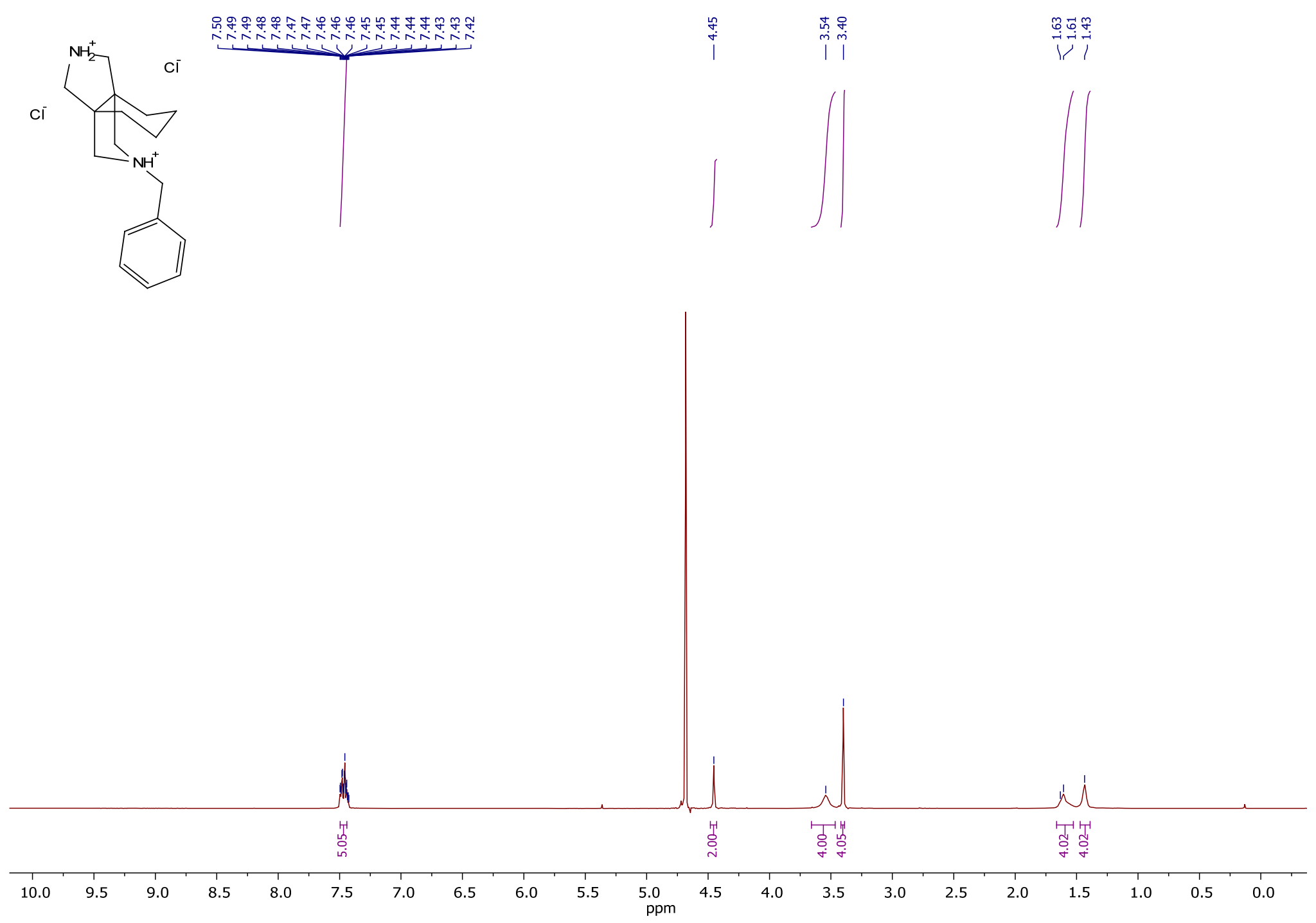

cis-2-benzylhexahydro-1H-3a,7a-(methanoiminomethano)isoindole (36) ${ }^{1} \mathrm{H}$ NMR 


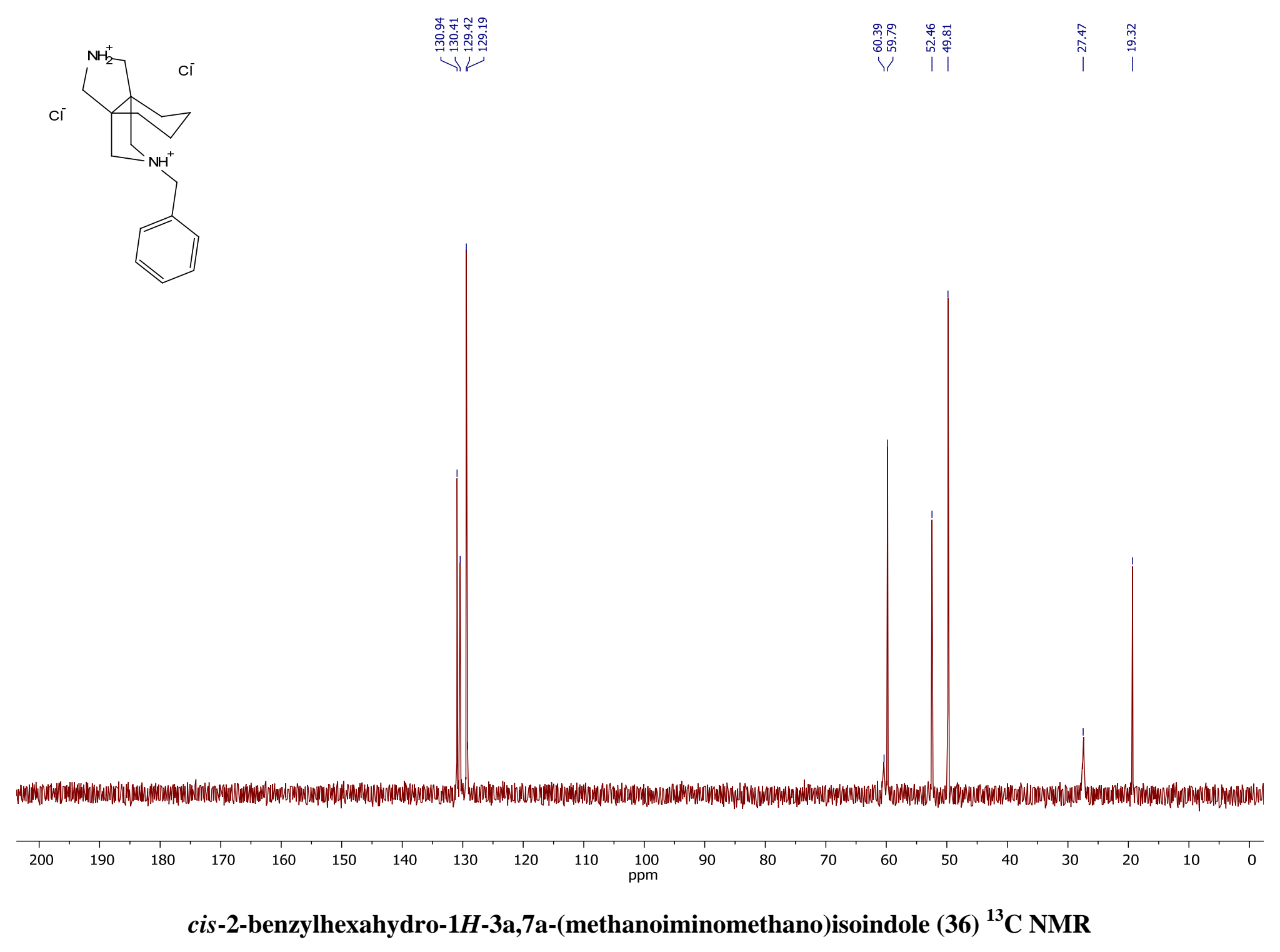



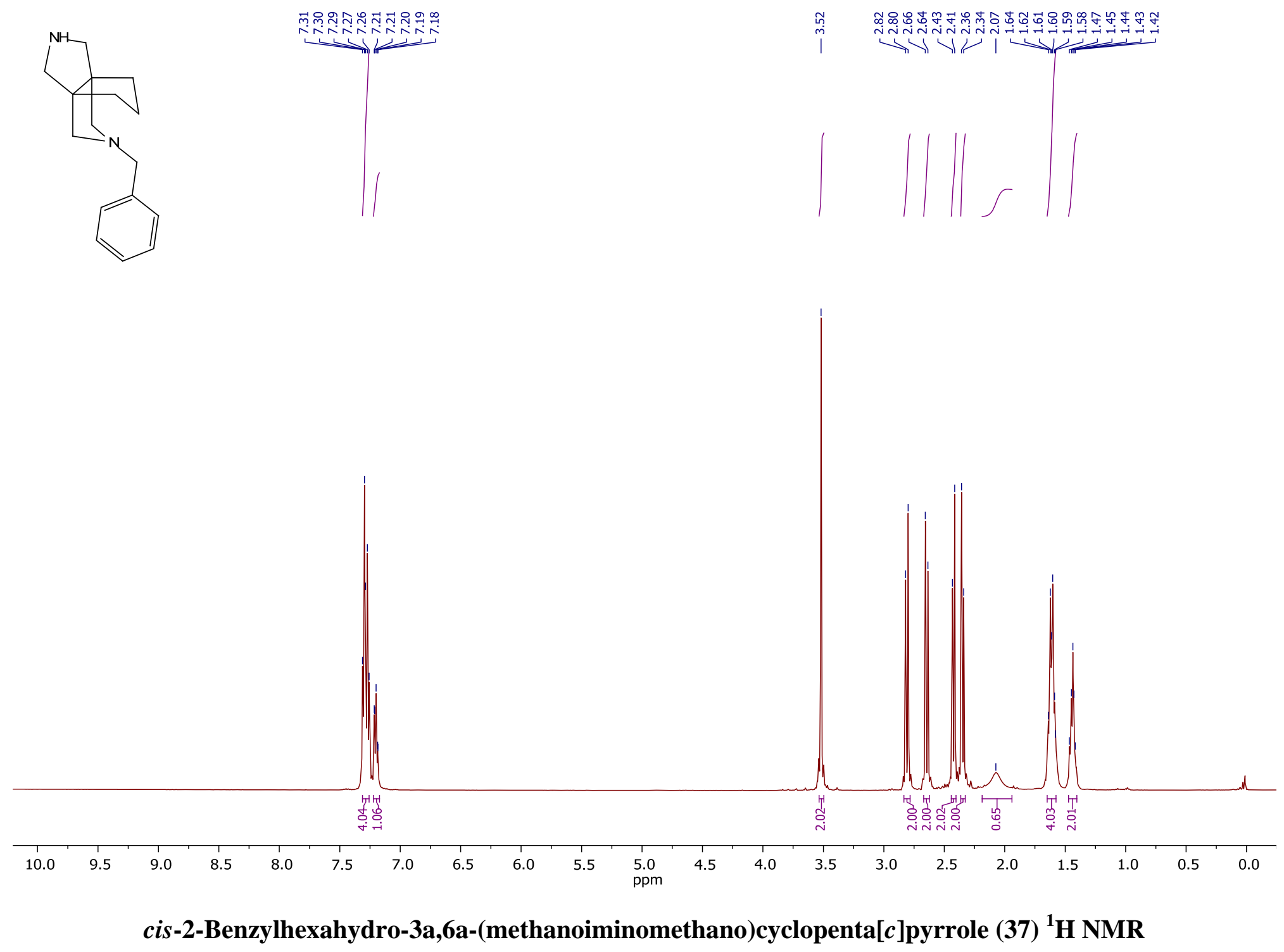


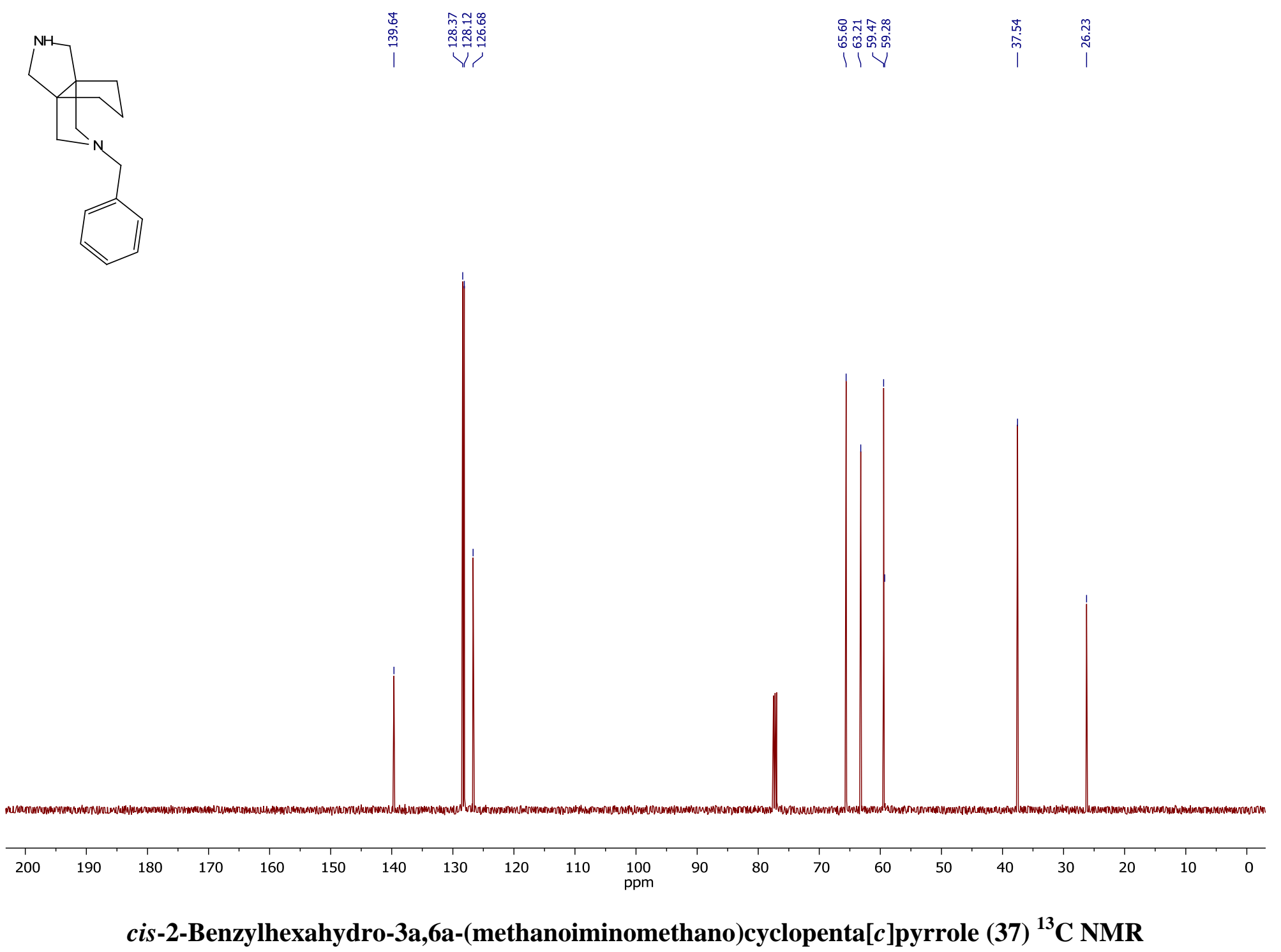



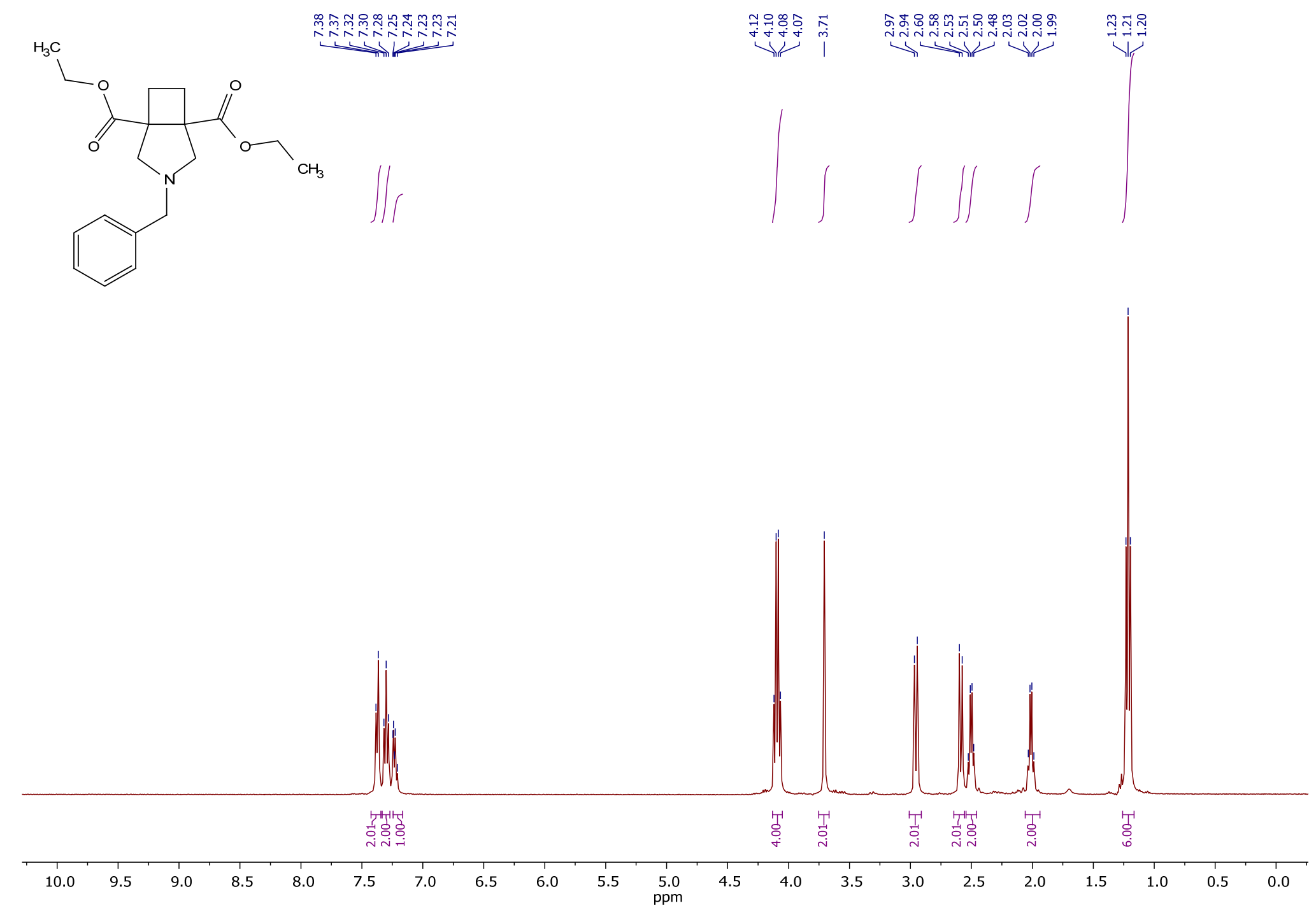

Diethyl 3-benzyl-3-azabicyclo[3.2.0]heptane-1,5-dicarboxylate (41) ${ }^{1}$ H NMR 


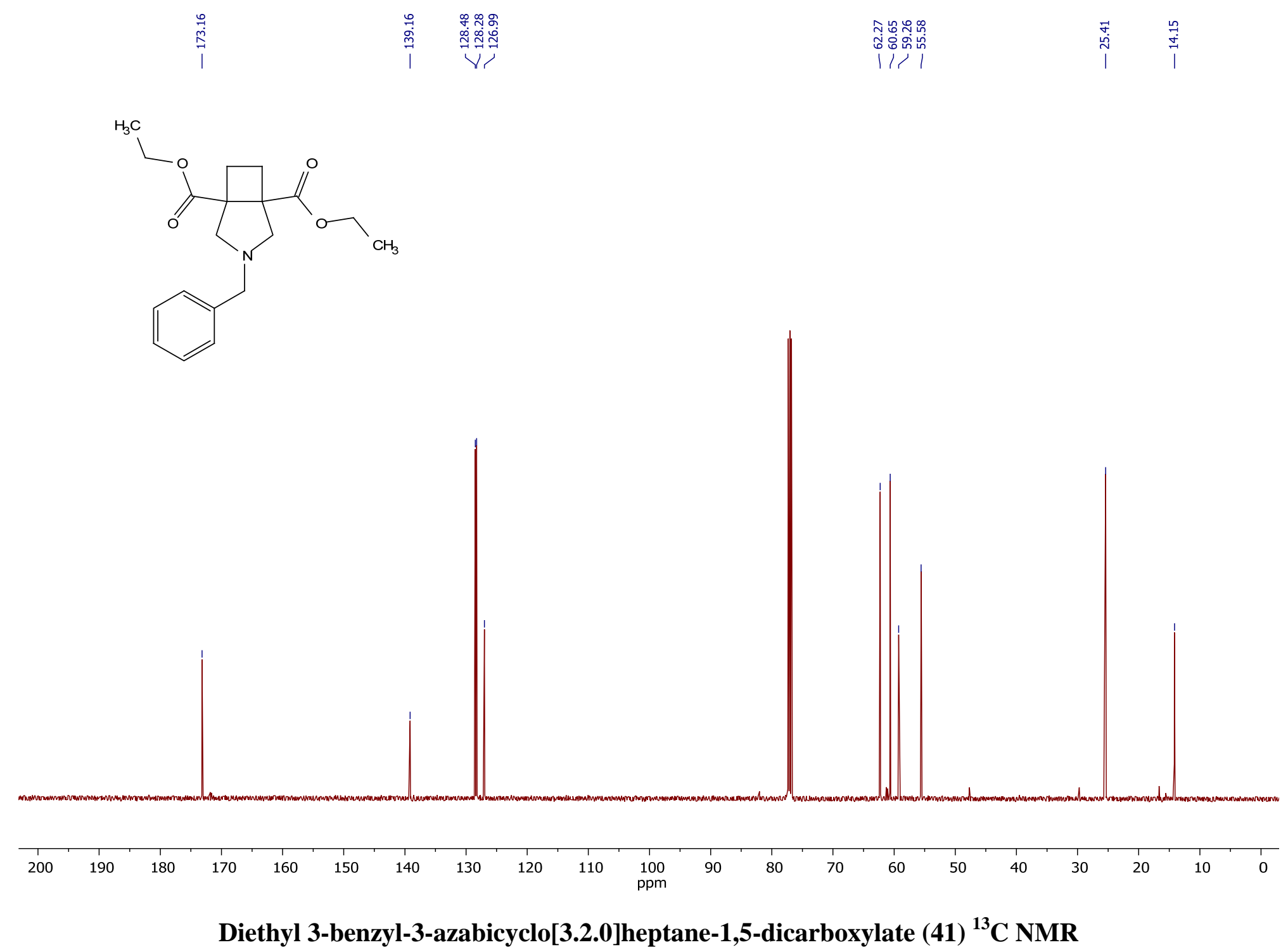




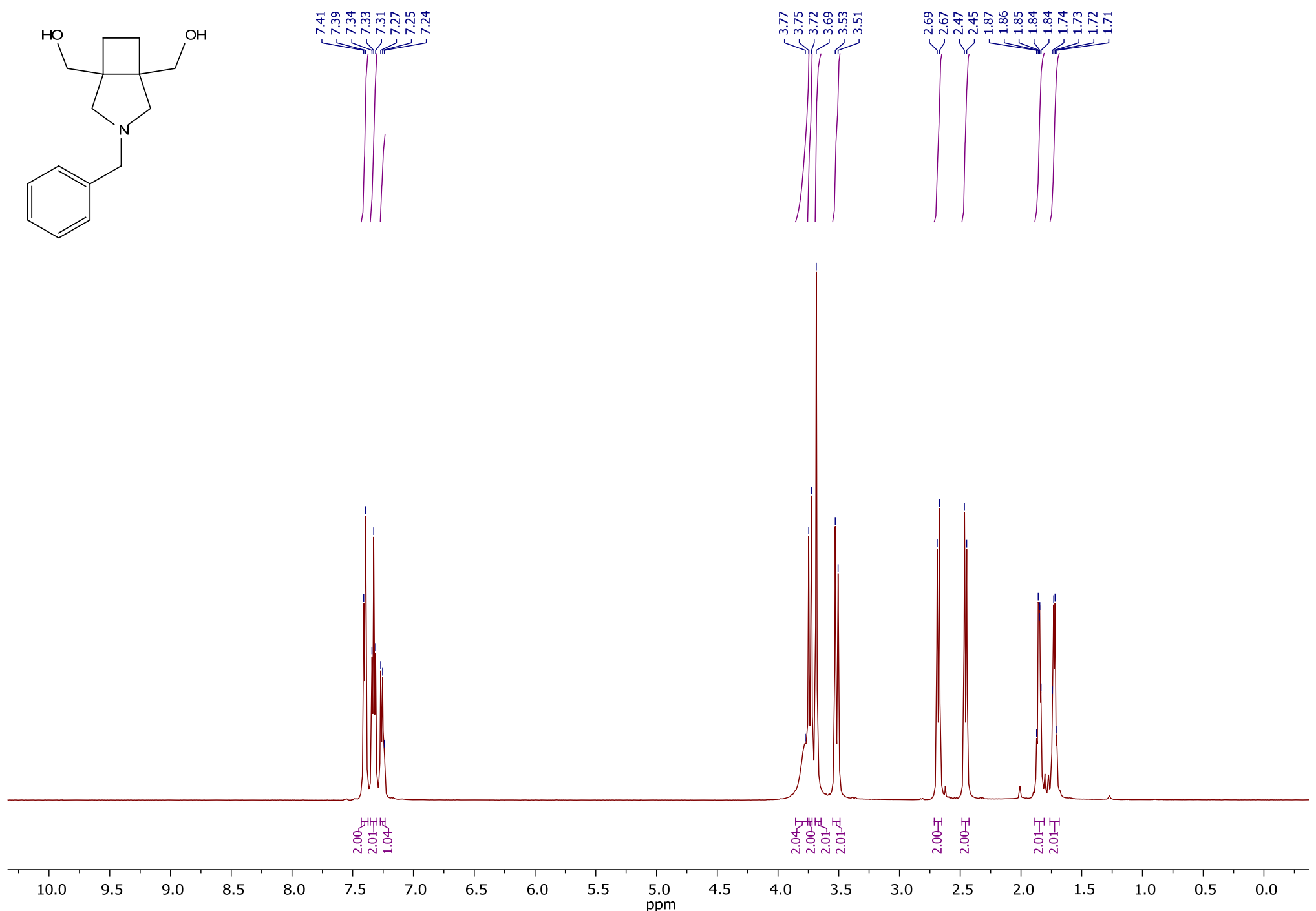

3-Benzyl-3-azabicyclo[3.2.0]heptane-1,5-diyl)dimethanol (42) ${ }^{1}$ H NMR 


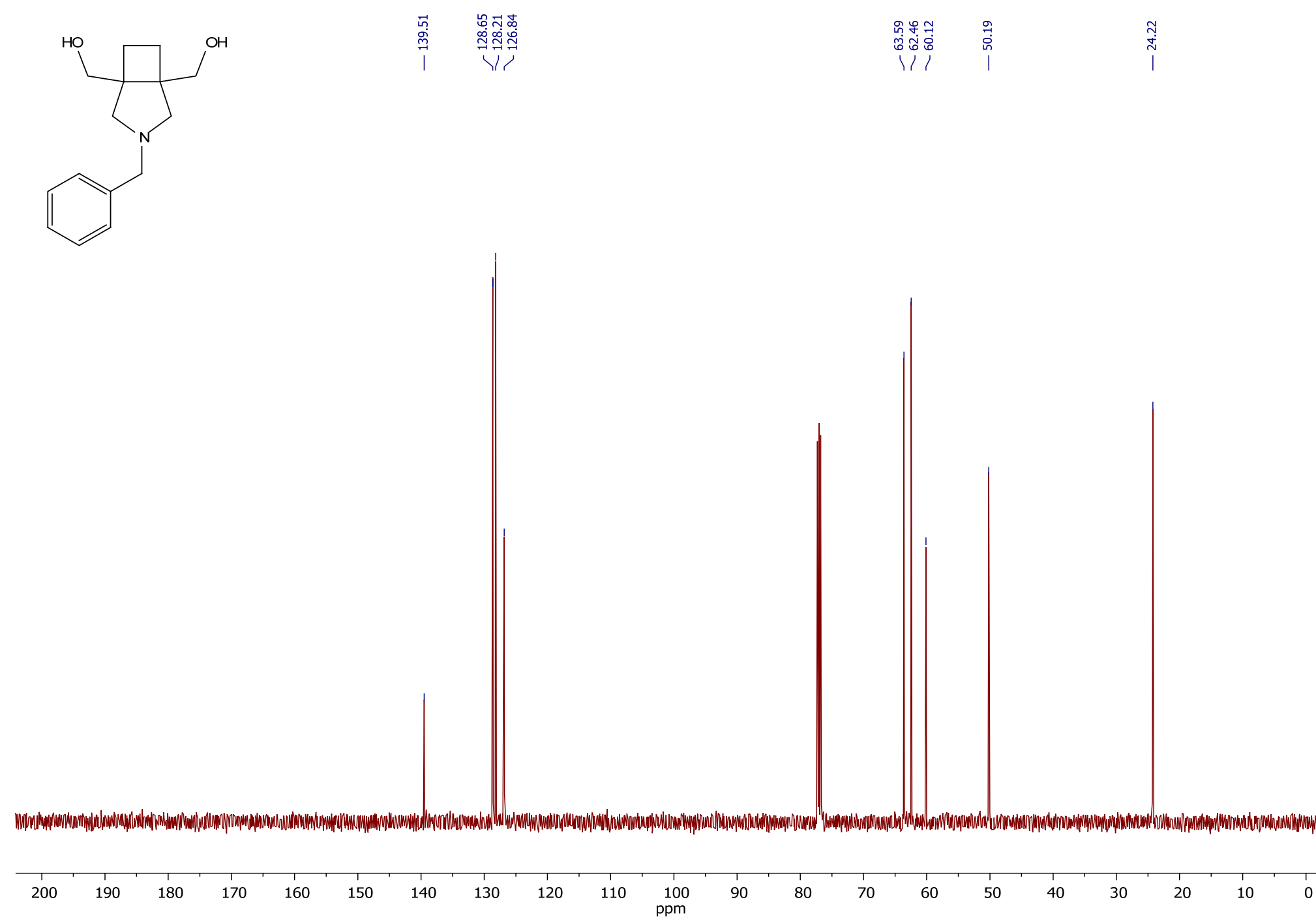

3-Benzyl-3-azabicyclo[3.2.0]heptane-1,5-diyl)dimethanol (42) ${ }^{13} \mathrm{C}$ NMR 


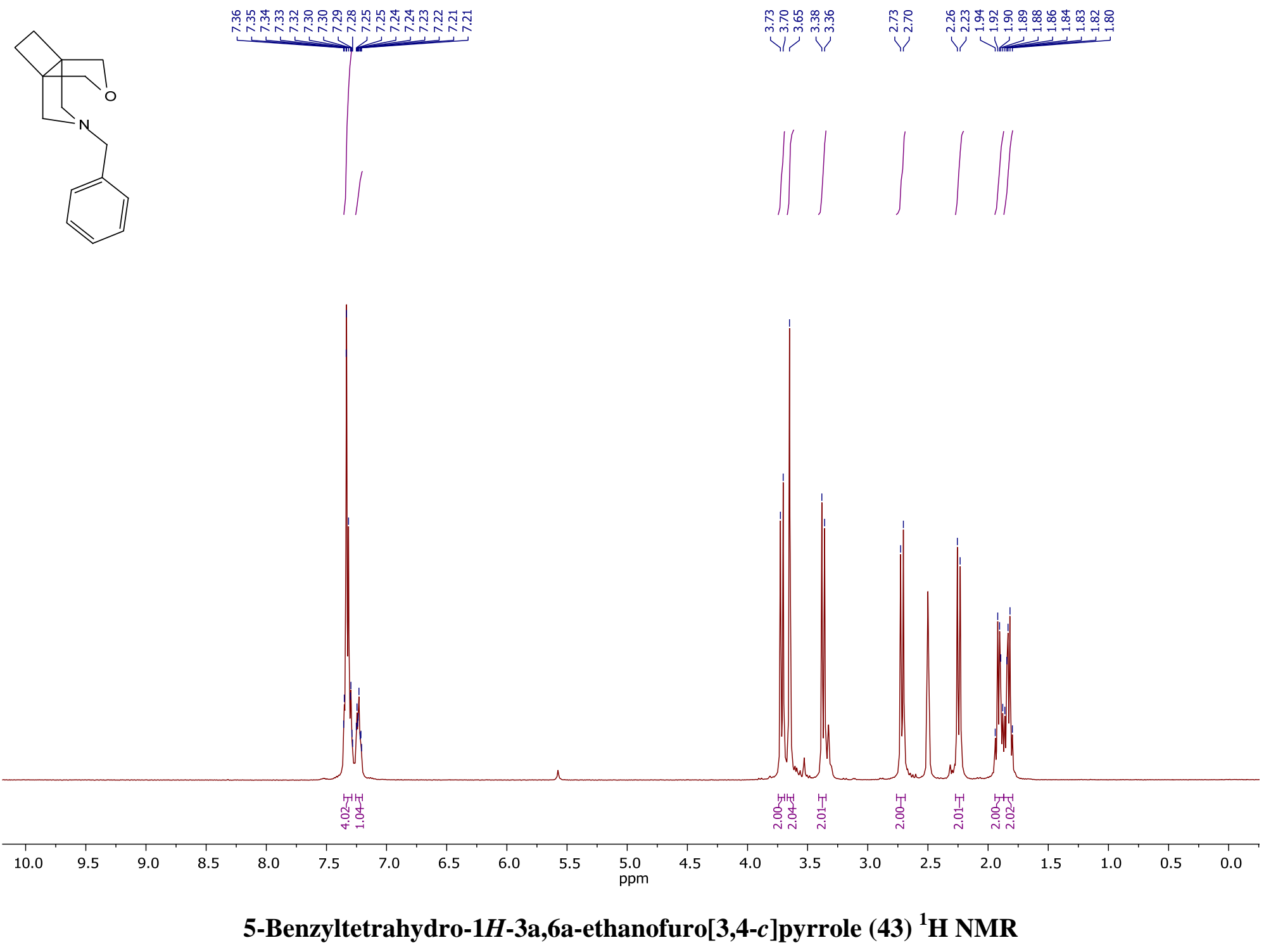



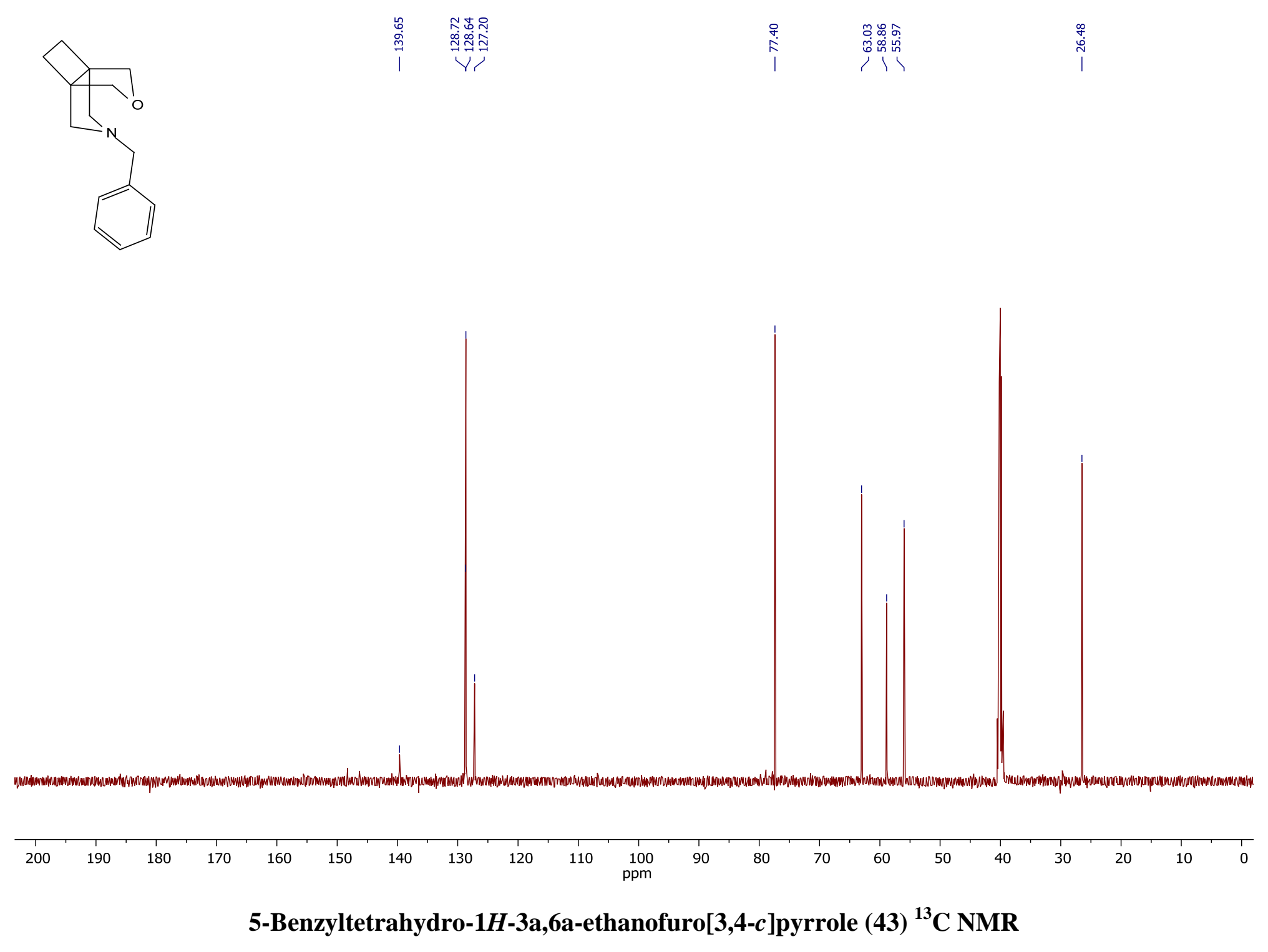


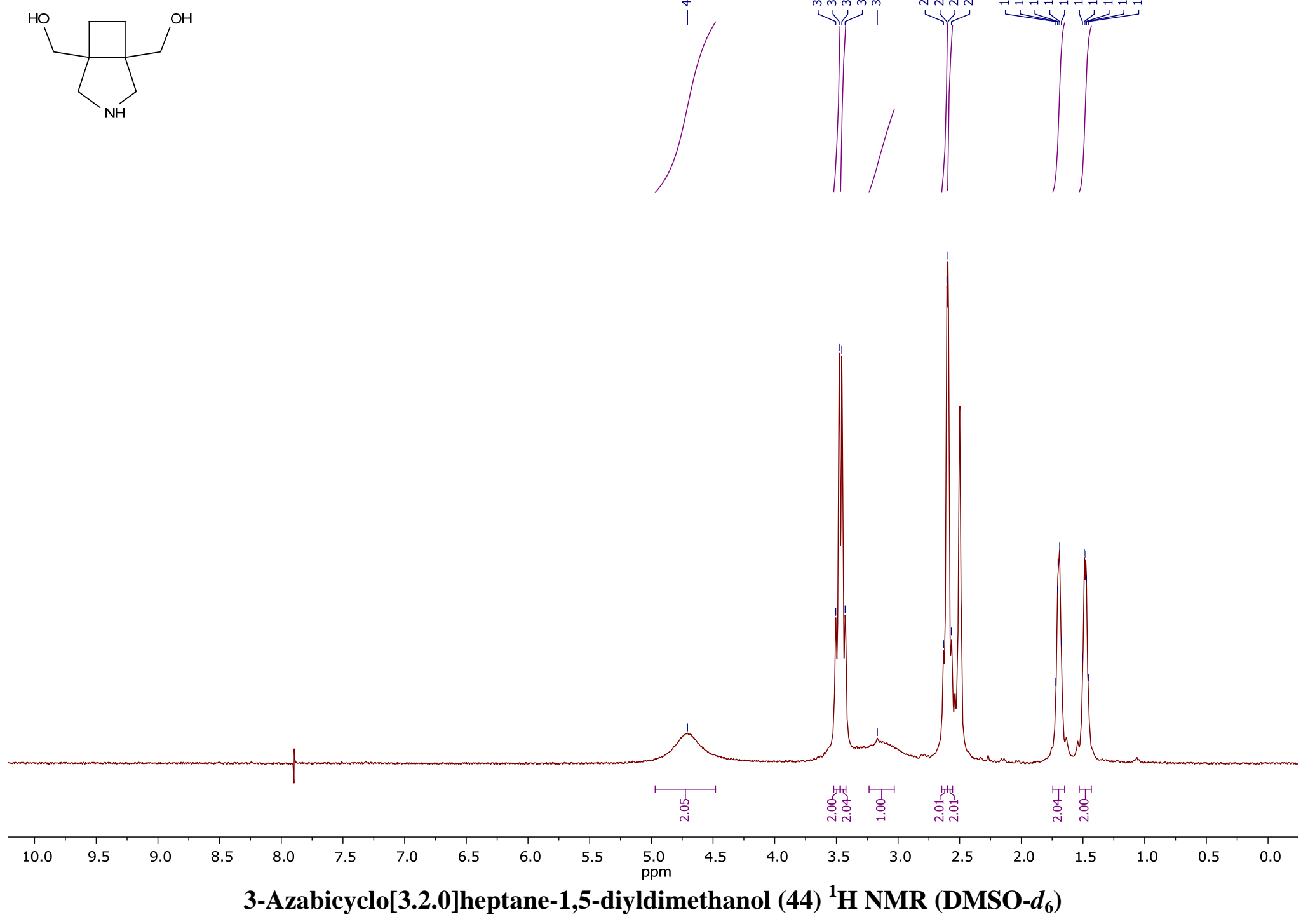



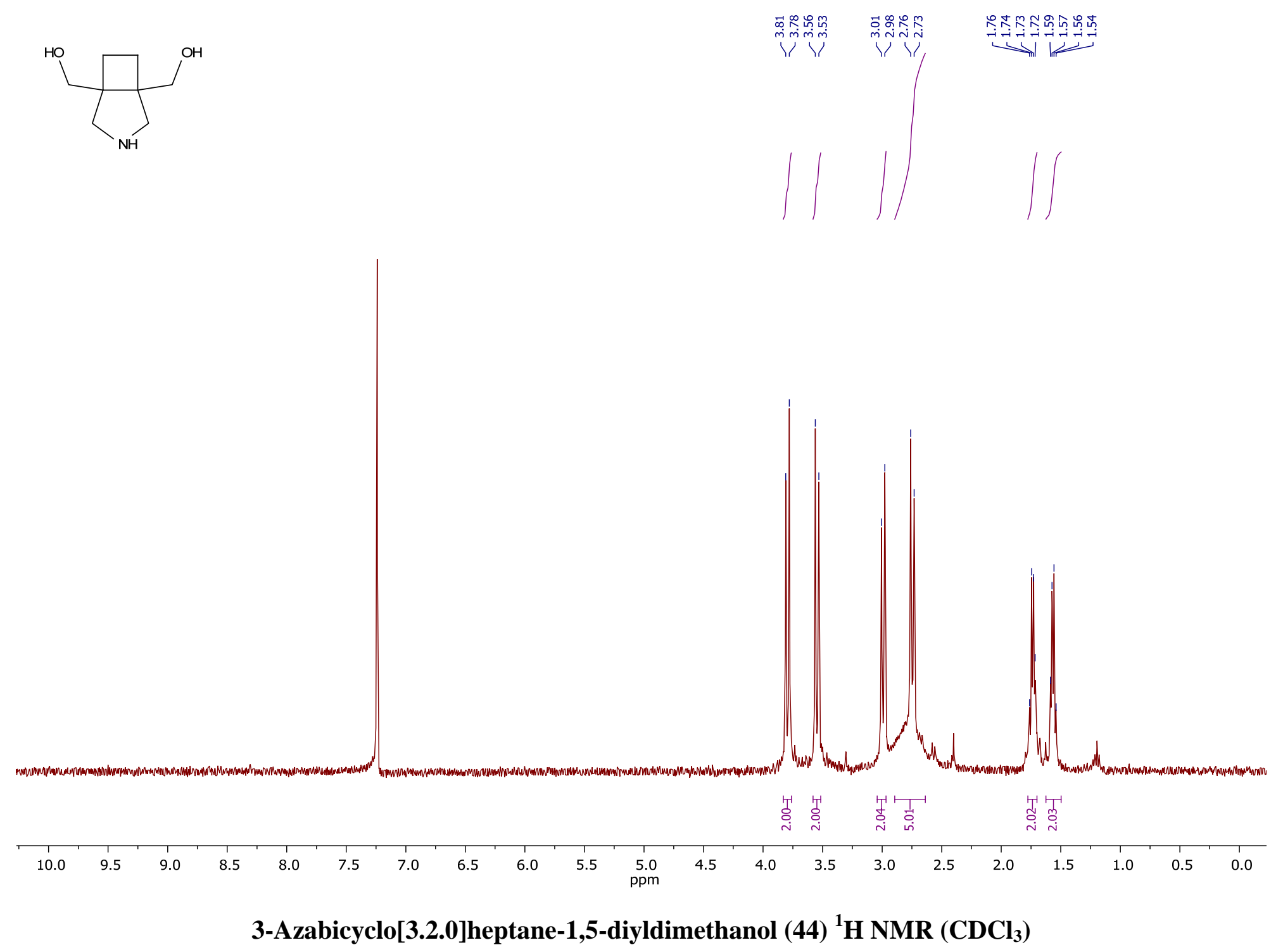

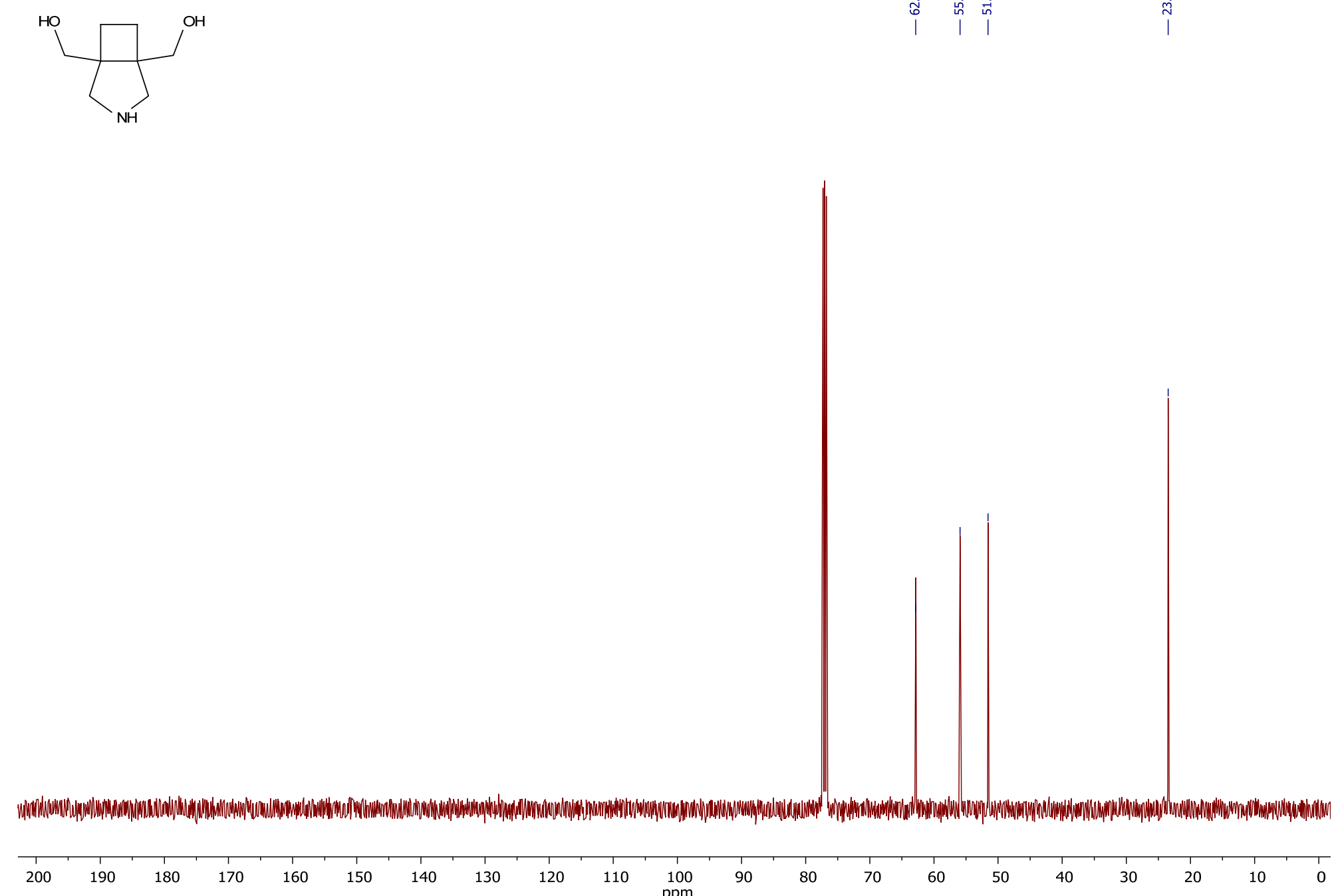

3-Azabicyclo[3.2.0]heptane-1,5-diyldimethanol (44) ${ }^{13} \mathrm{C}$ NMR $\left(\mathrm{CDCl}_{3}\right)$ 


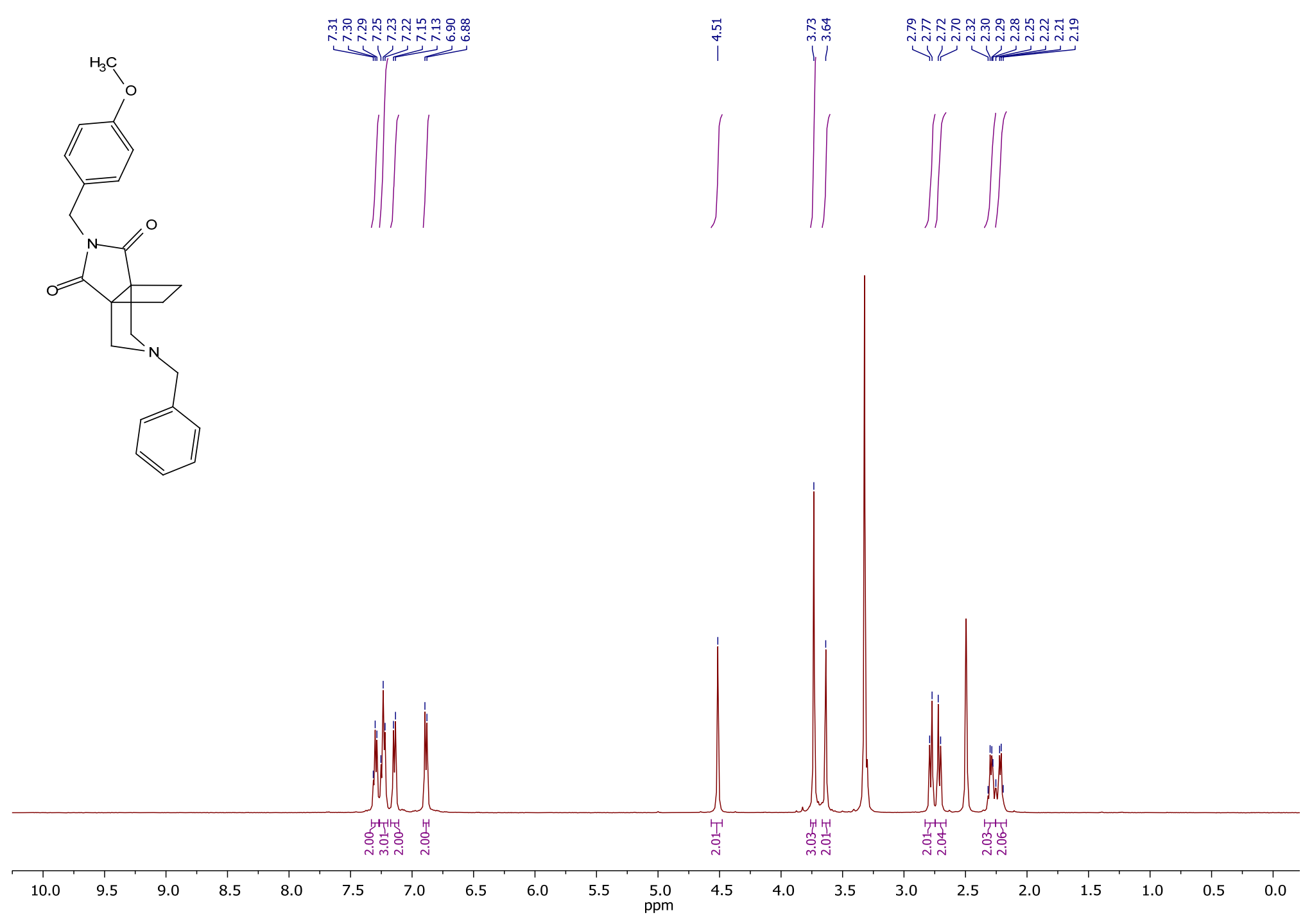

5-Benzyl-2-(4-methoxybenzyl)dihydro-3a,6a-ethanopyrrolo[3,4-c]pyrrole-1,3(2H,4H)-dione (45) ${ }^{1} \mathrm{H}$ NMR 


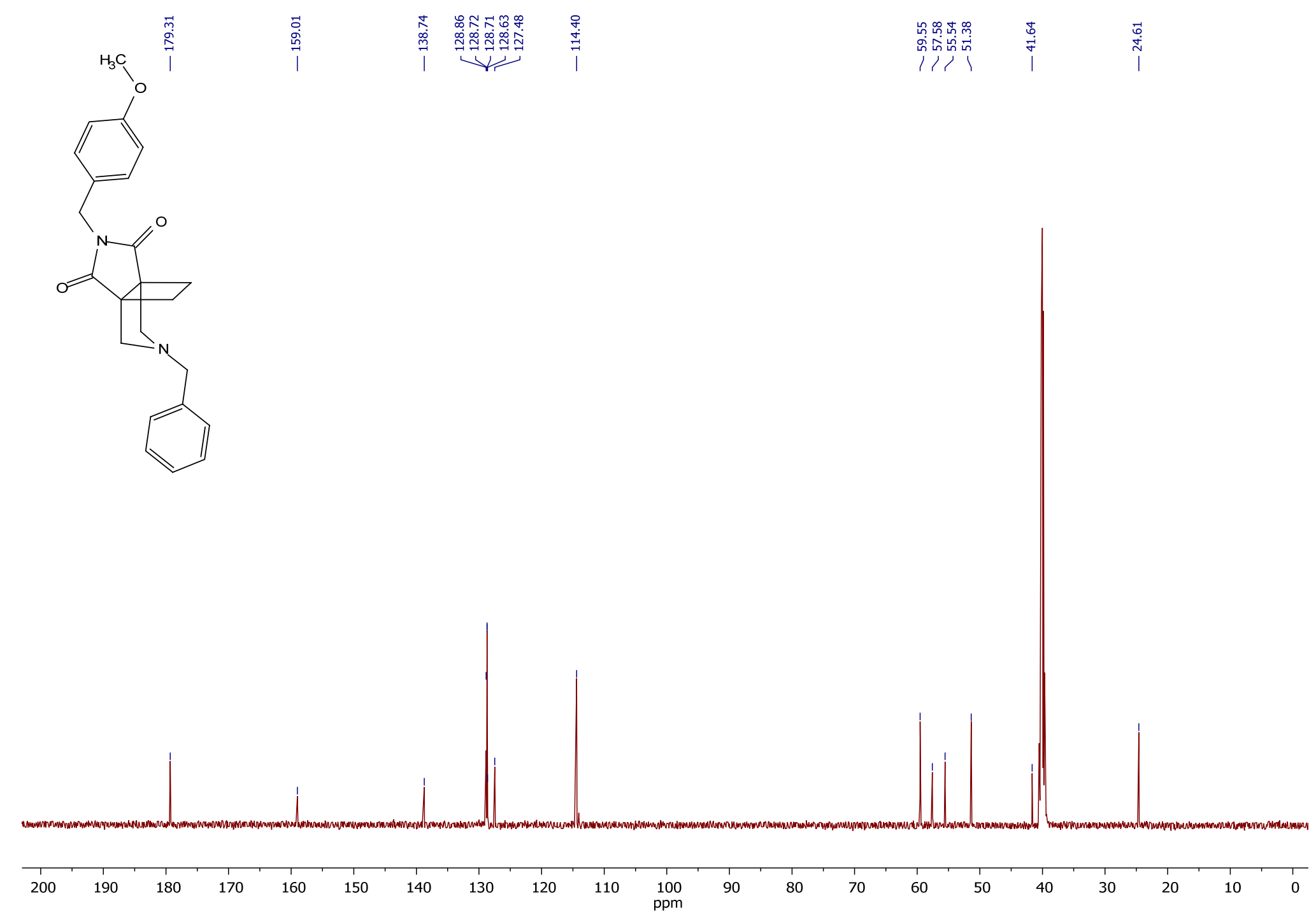

5-Benzyl-2-(4-methoxybenzyl)dihydro-3a,6a-ethanopyrrolo[3,4-c]pyrrole-1,3(2H,4H)-dione (45) ${ }^{13} \mathrm{C}$ NMR 


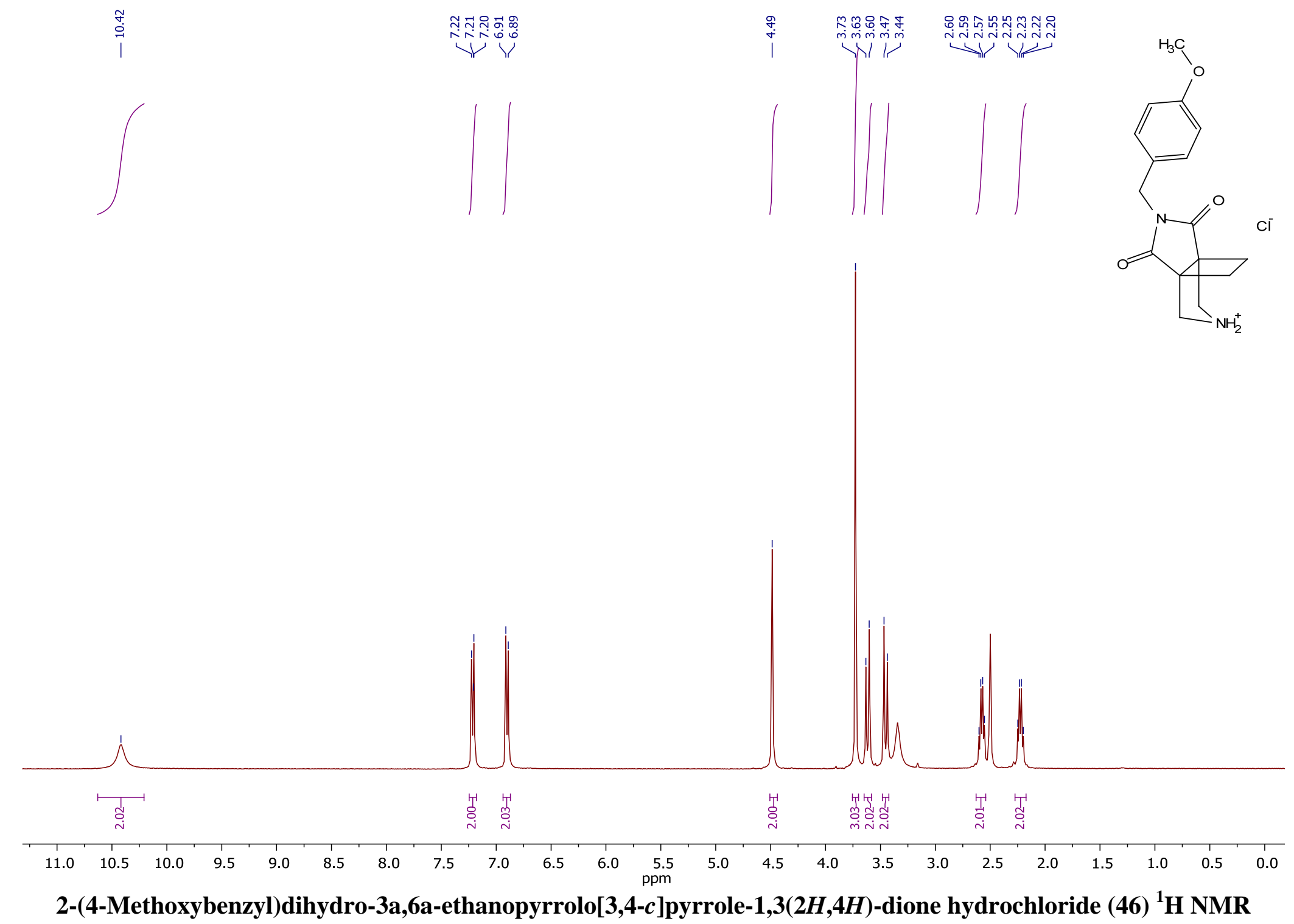



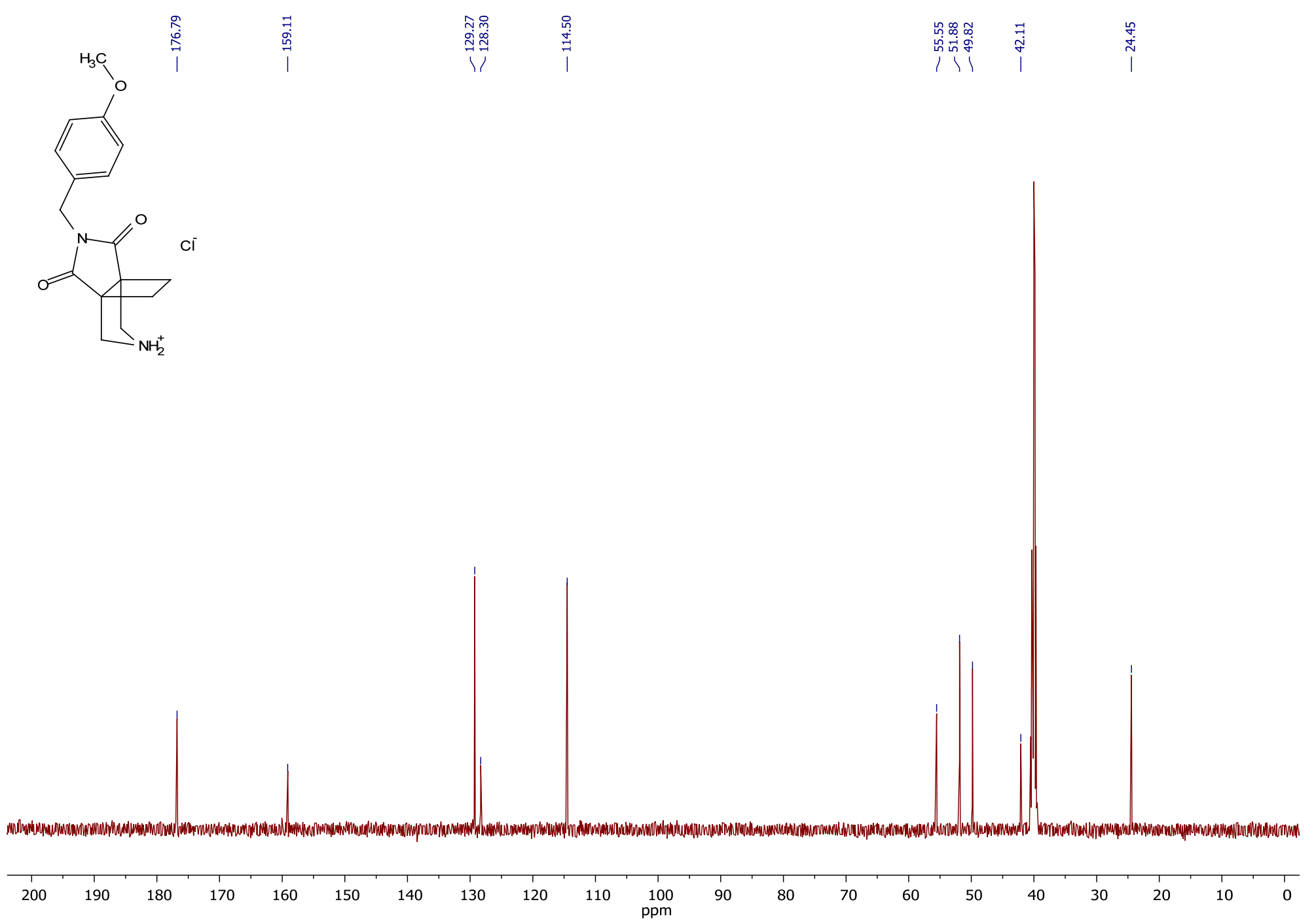

2-(4-Methoxybenzyl)dihydro-3a,6a-ethanopyrrolo[3,4-c]pyrrole-1,3(2H,4H)-dione hydrochloride (46) ${ }^{13} \mathrm{C}$ NMR 


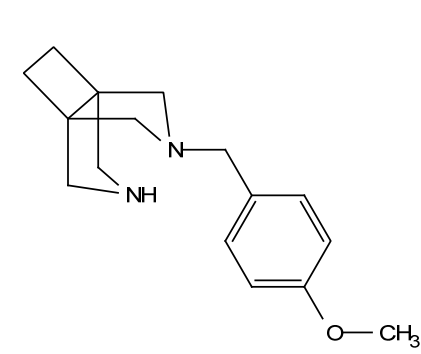

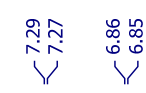

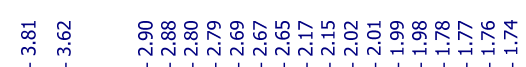

|
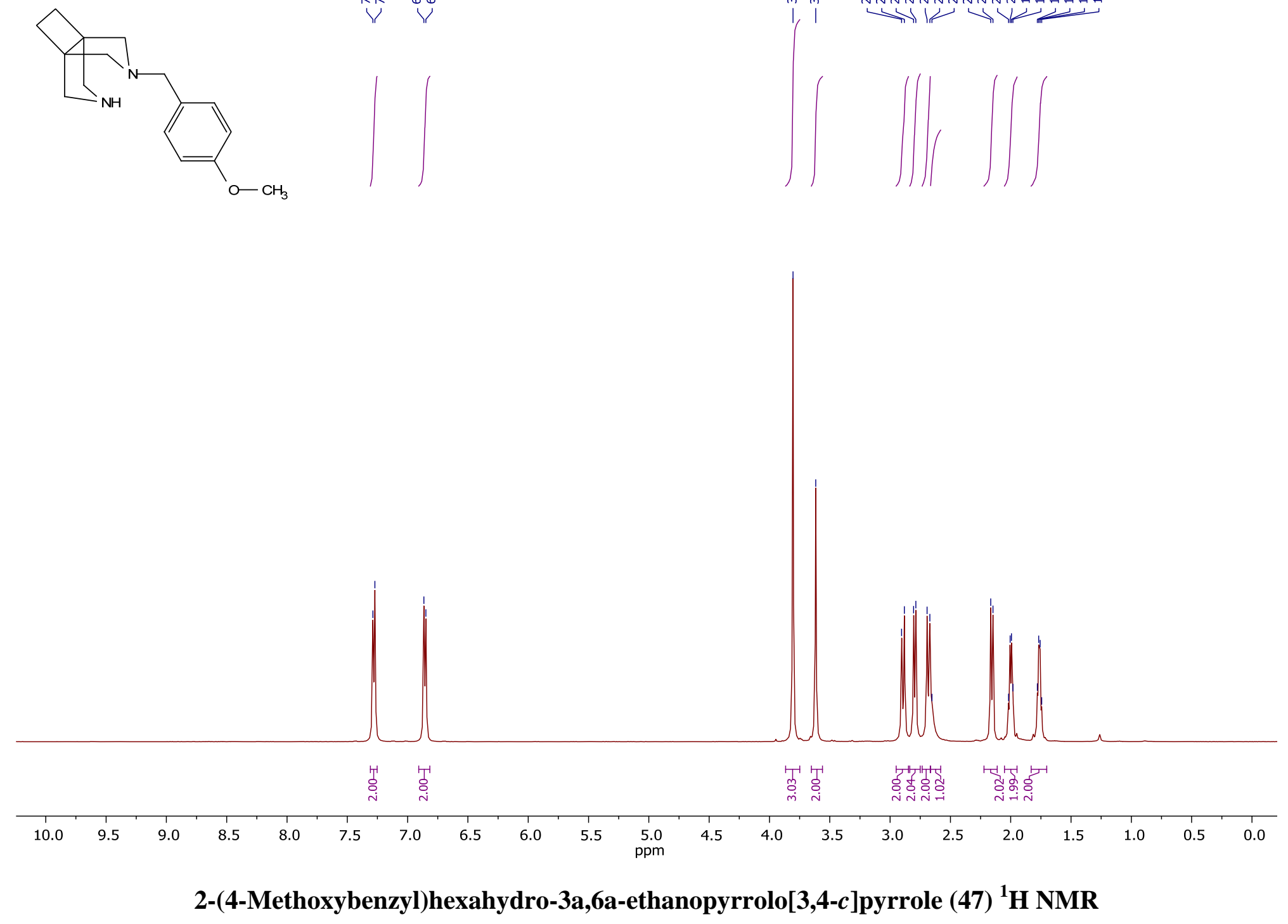


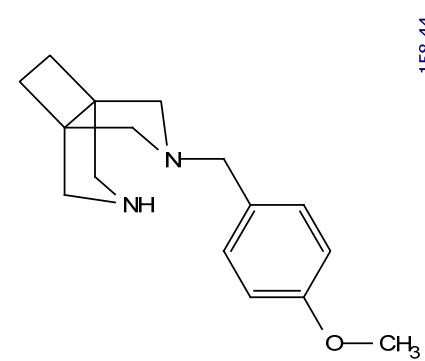

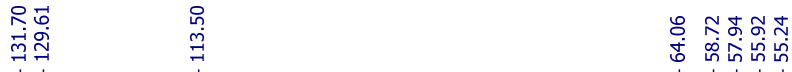

审

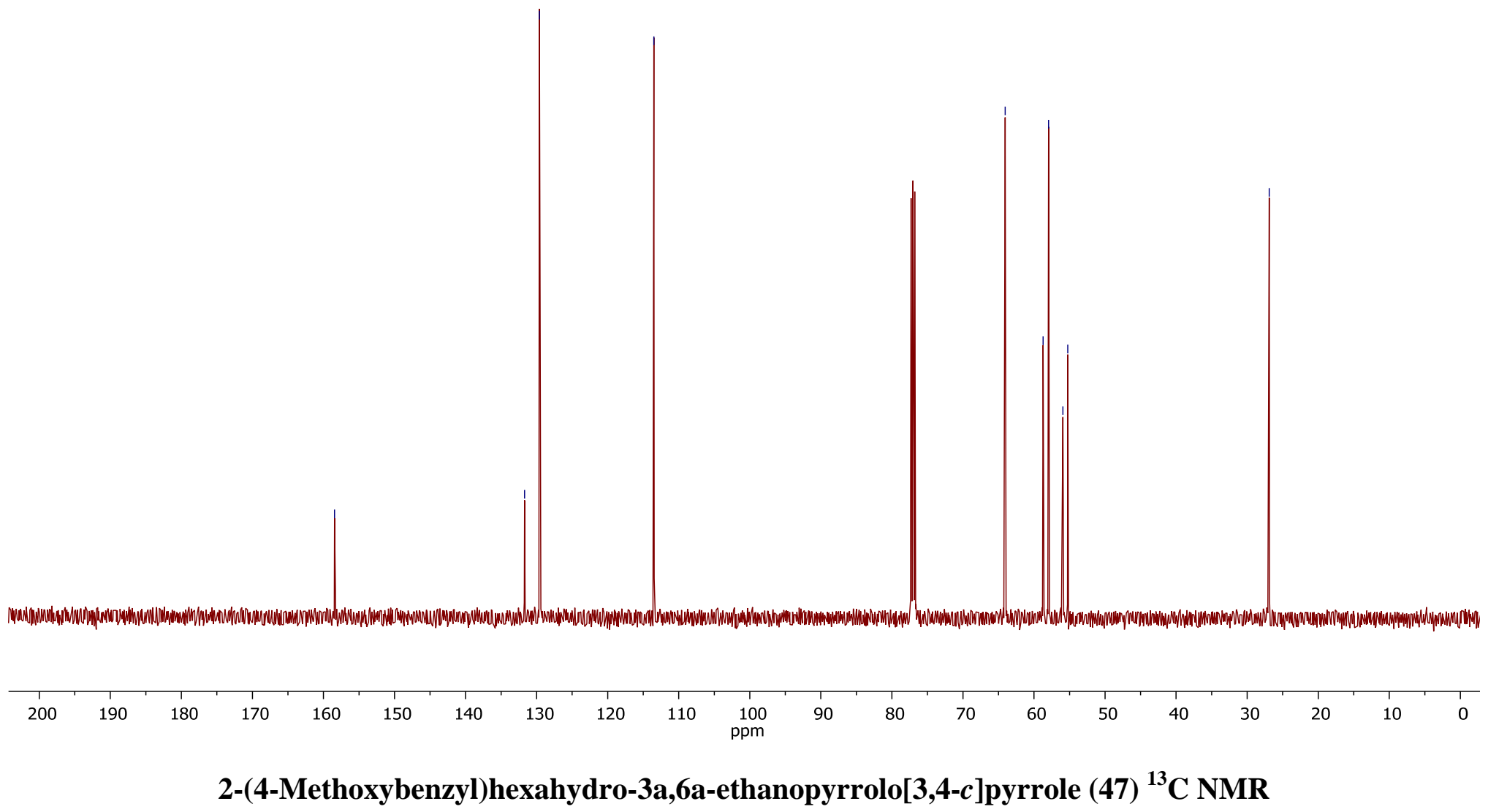



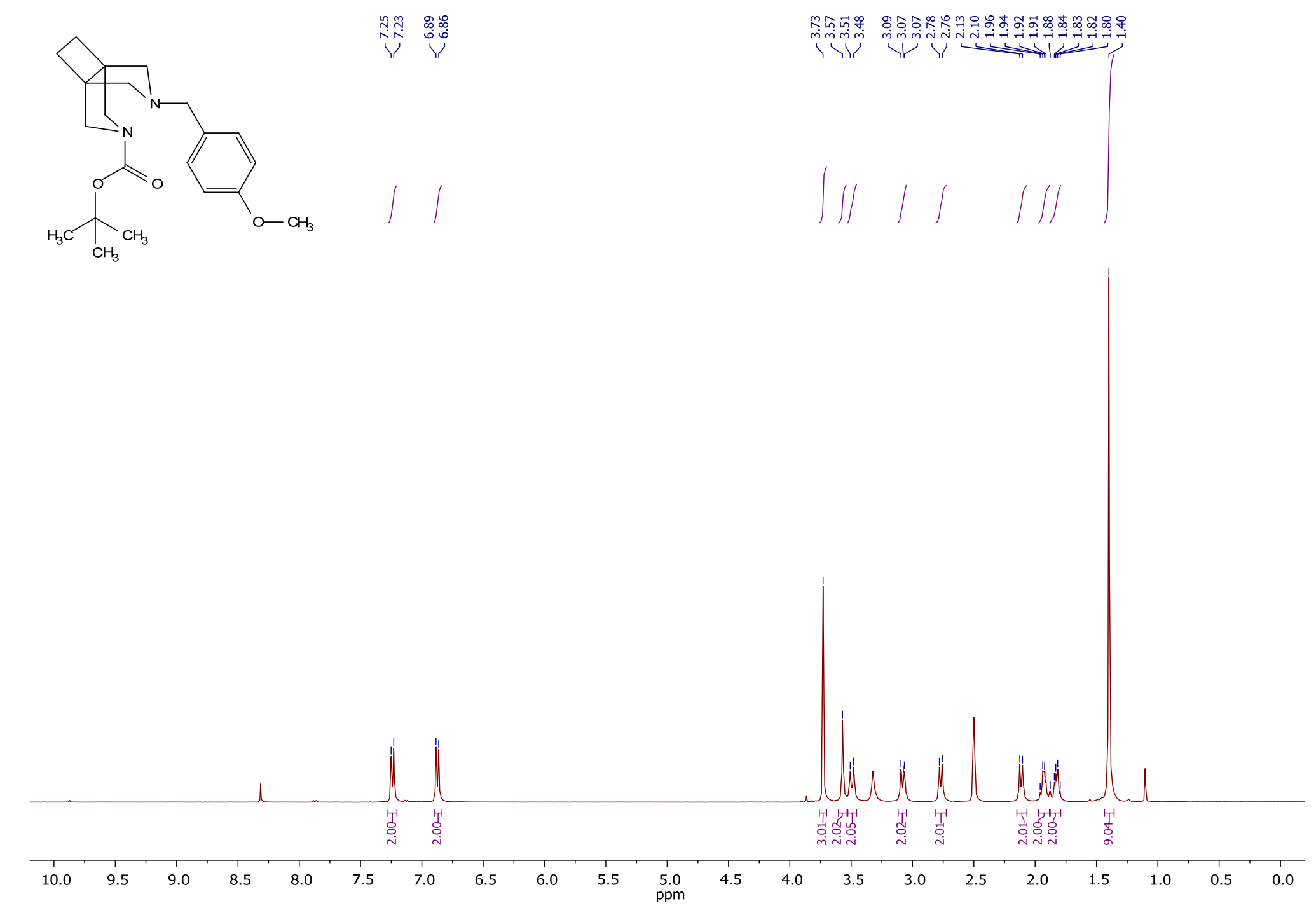

tert-Butyl 5-(4-methoxybenzyl)tetrahydro-3a,6a-ethanopyrrolo[3,4-c]pyrrole-2(1H)-carboxylate (48) ${ }^{1} \mathrm{H}$ NMR 


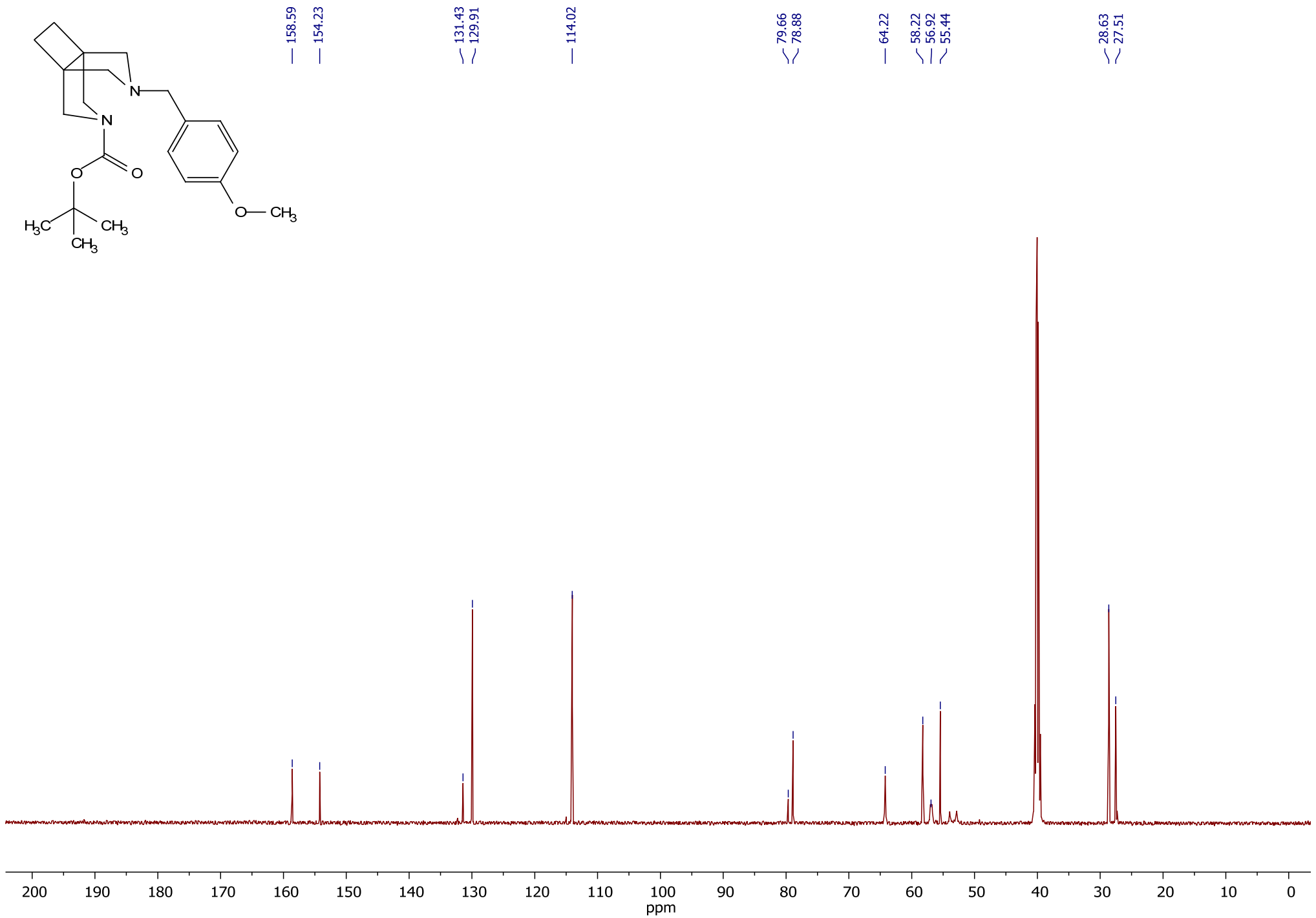

tert-Butyl 5-(4-methoxybenzyl)tetrahydro-3a,6a-ethanopyrrolo[3,4-c]pyrrole-2(1H)-carboxylate (48) ${ }^{13} \mathrm{C}$ NMR 


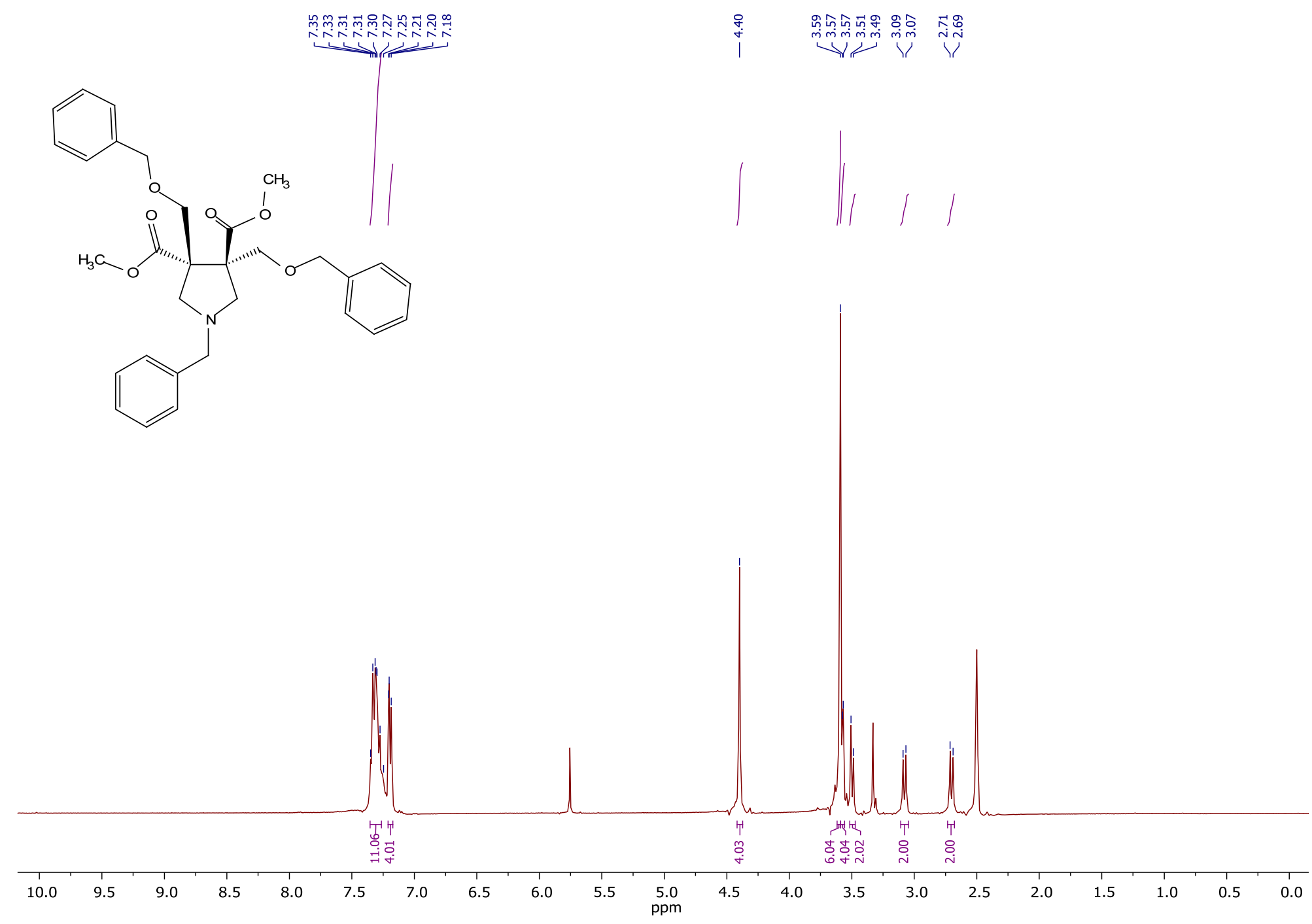

Dimethyl 1-benzyl-3,4-bis((benzyloxy)methyl)pyrrolidine-3,4-dicarboxylate (51) ${ }^{1} \mathrm{H}$ NMR 


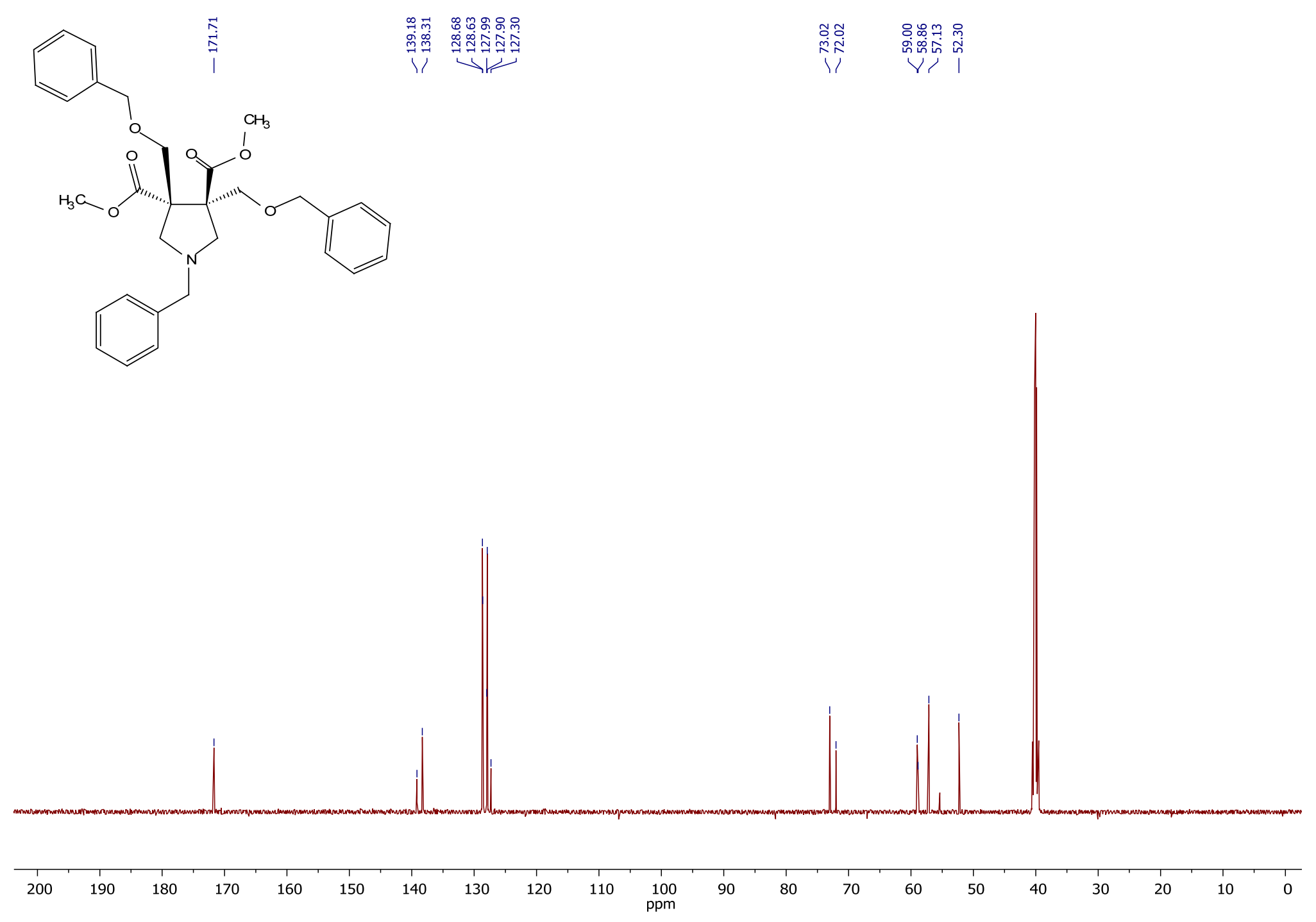

Dimethyl 1-benzyl-3,4-bis((benzyloxy)methyl)pyrrolidine-3,4-dicarboxylate $(51){ }^{13} \mathrm{C}$ NMR 


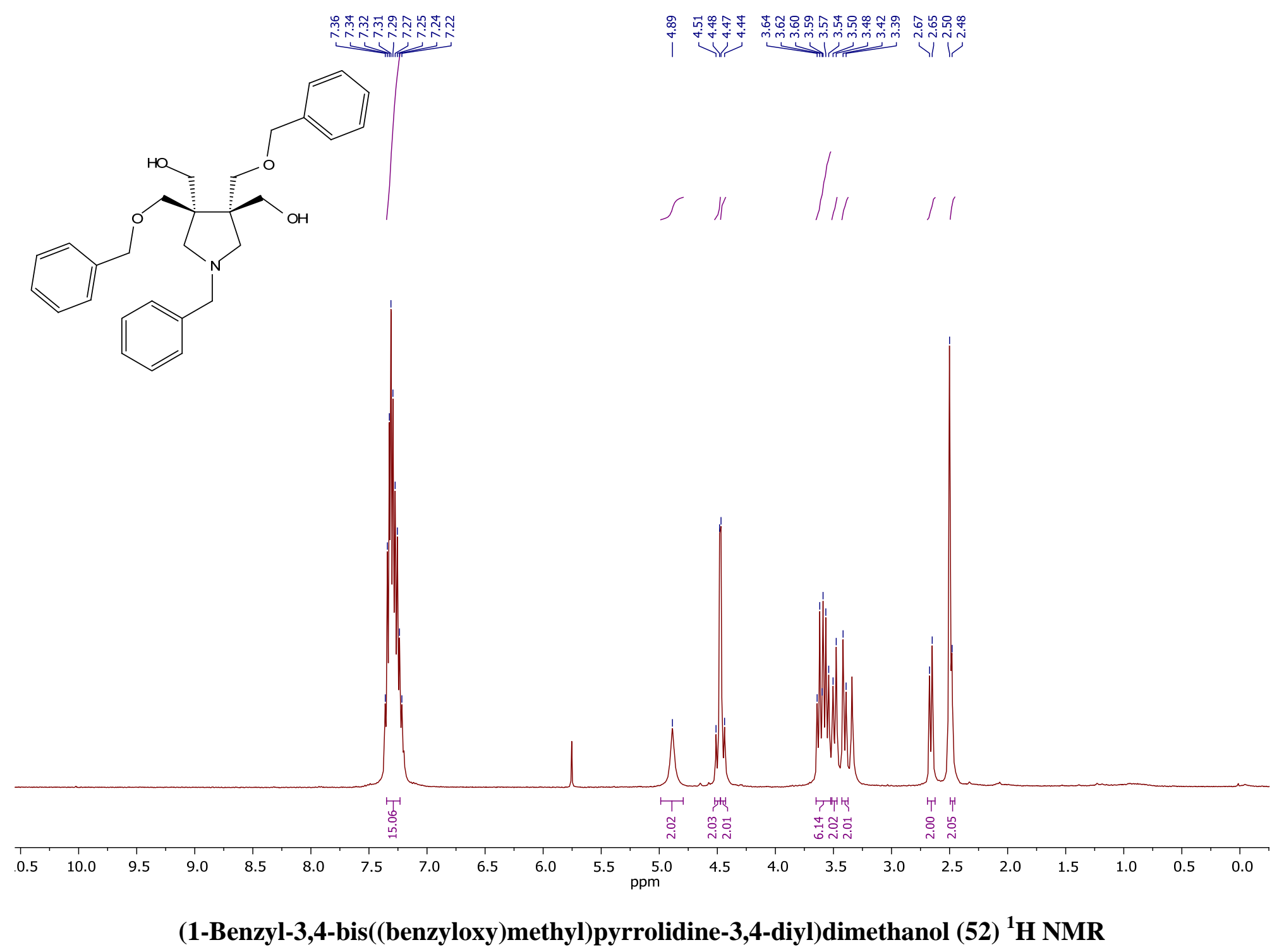




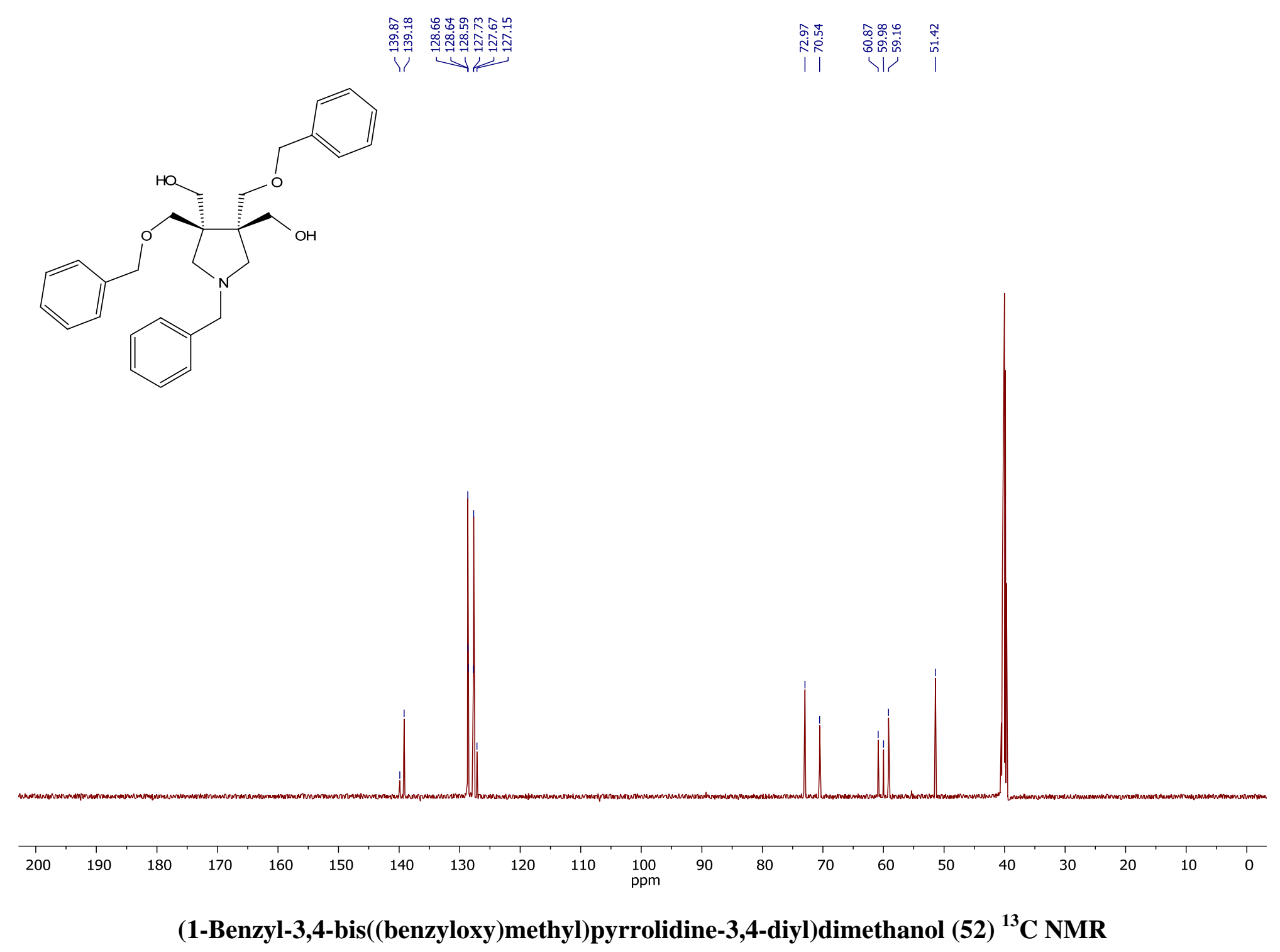




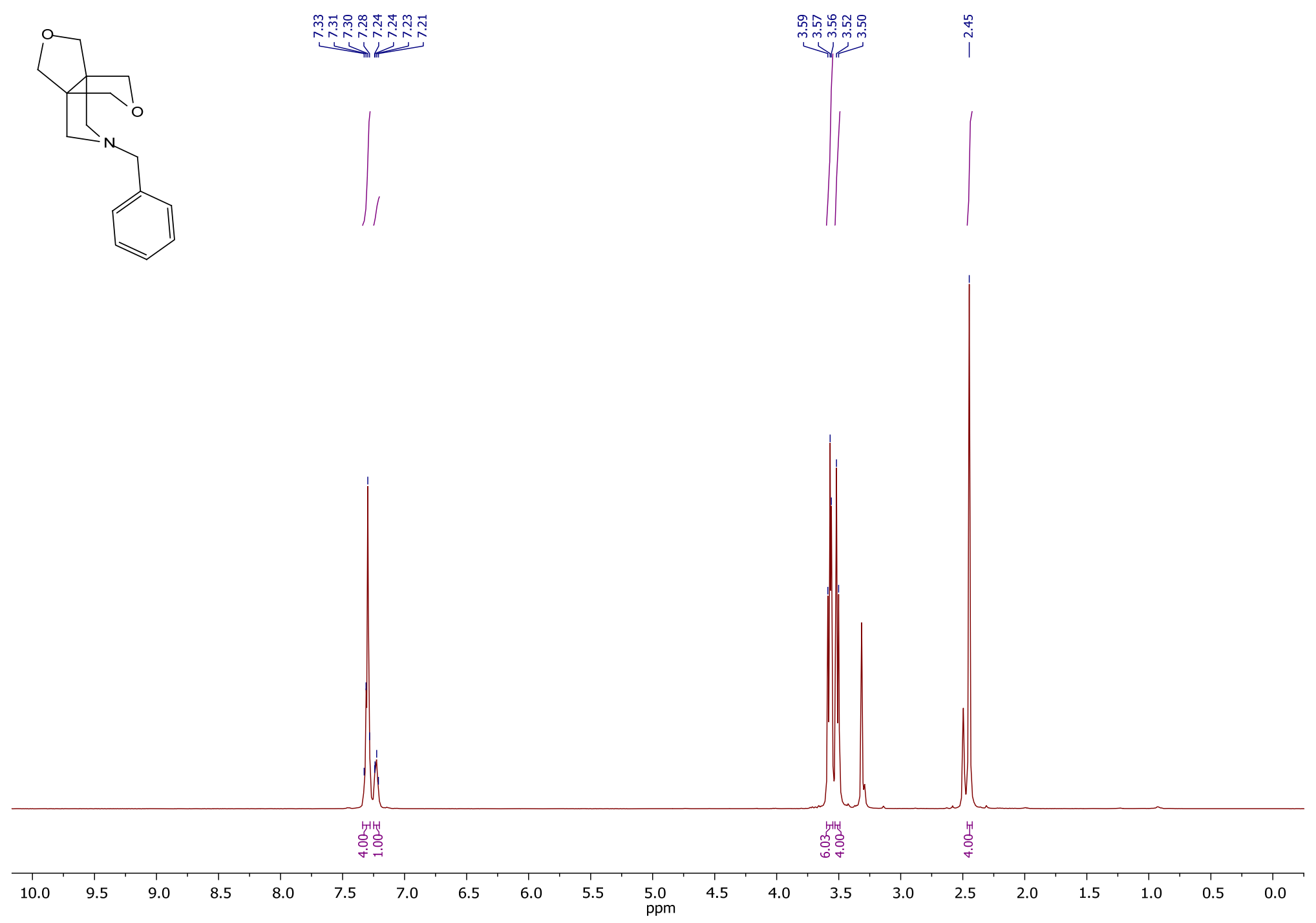

5-Benzyltetrahydro-1H-3a,6a-(methanooxymethano)furo[3,4-c]pyrrole (53) ${ }^{1} \mathrm{H}$ NMR 


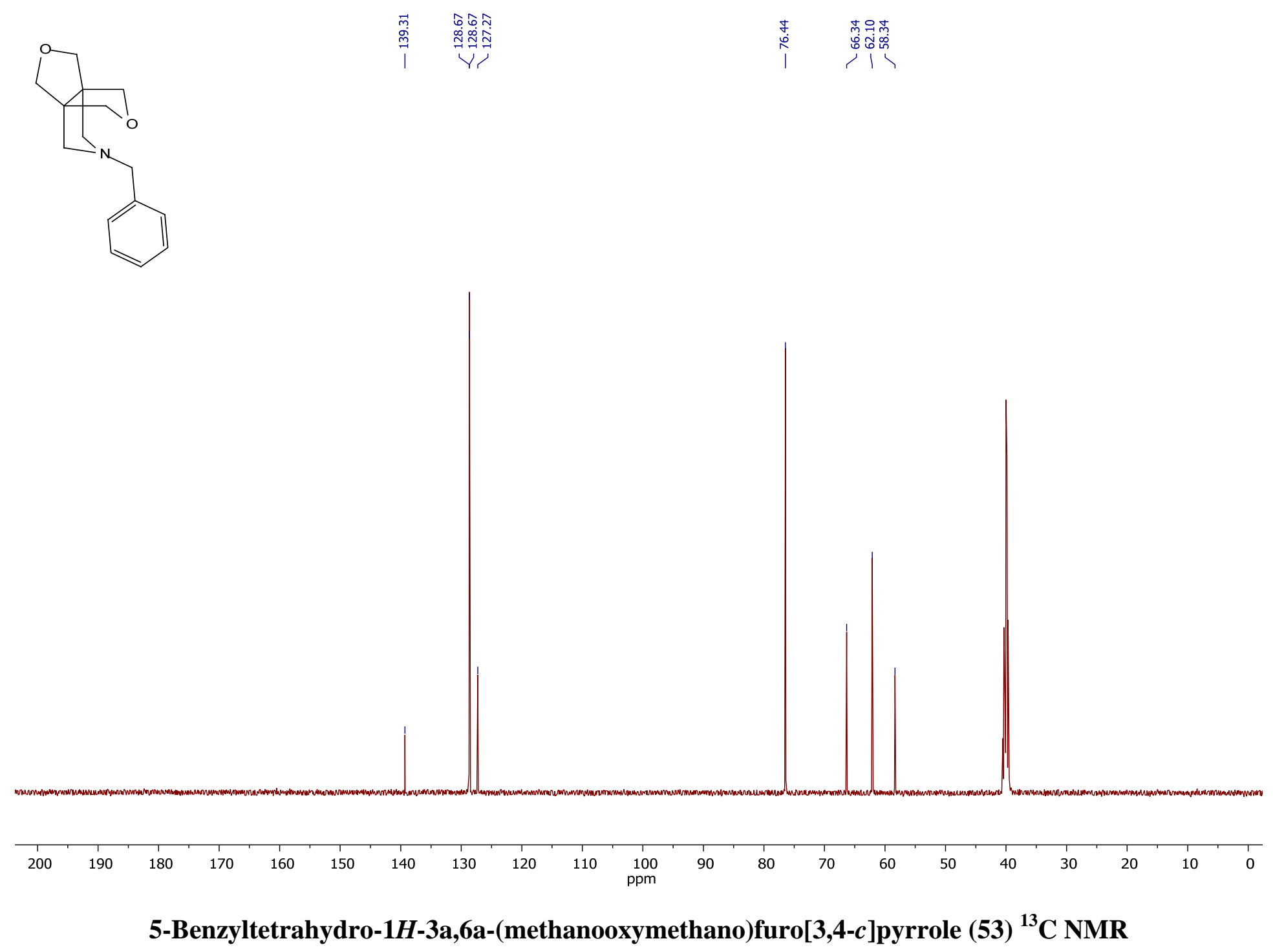




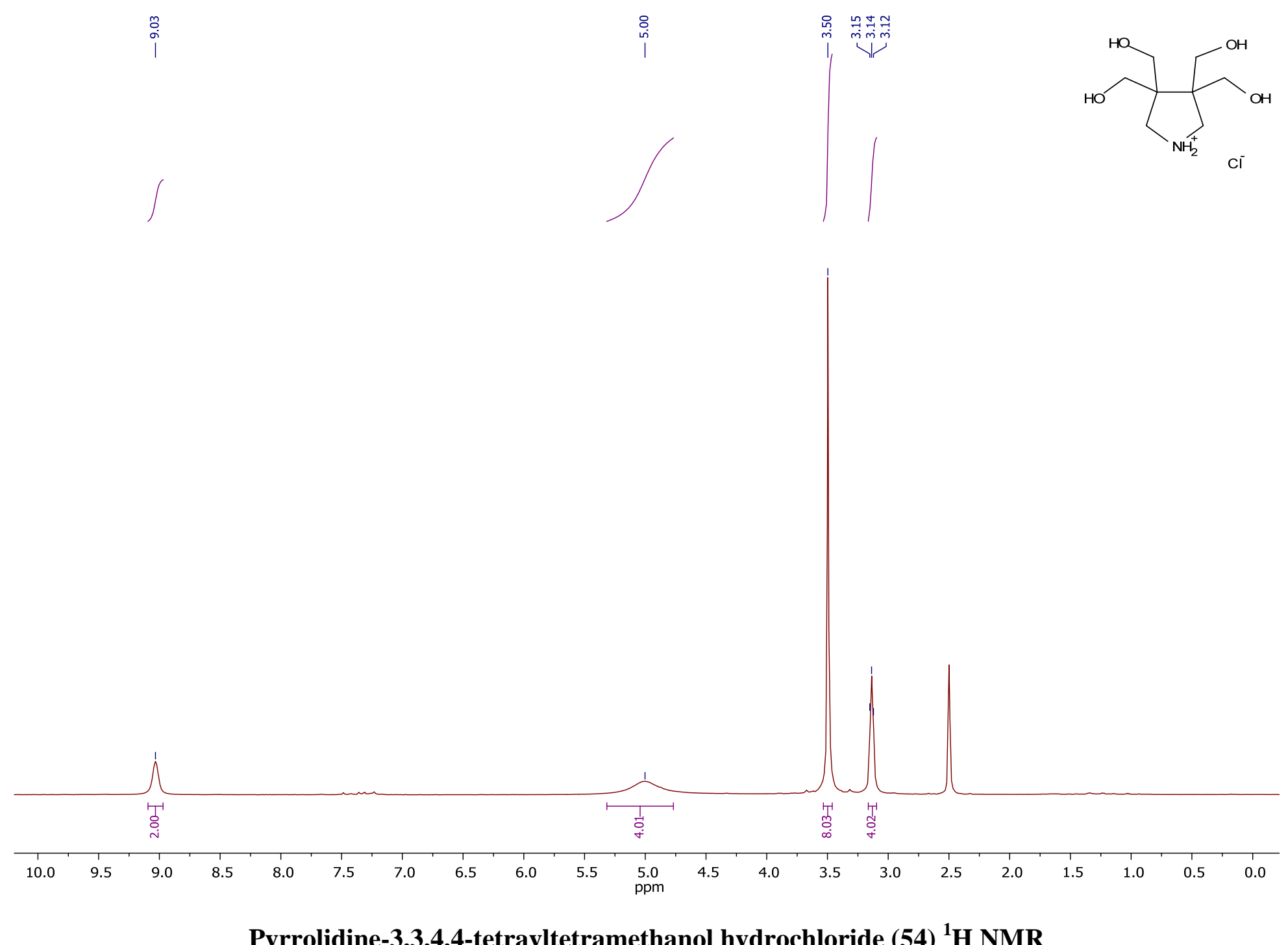

Pyrrolidine-3,3,4,4-tetrayltetramethanol hydrochloride (54) ${ }^{1} \mathrm{H}$ NMR 

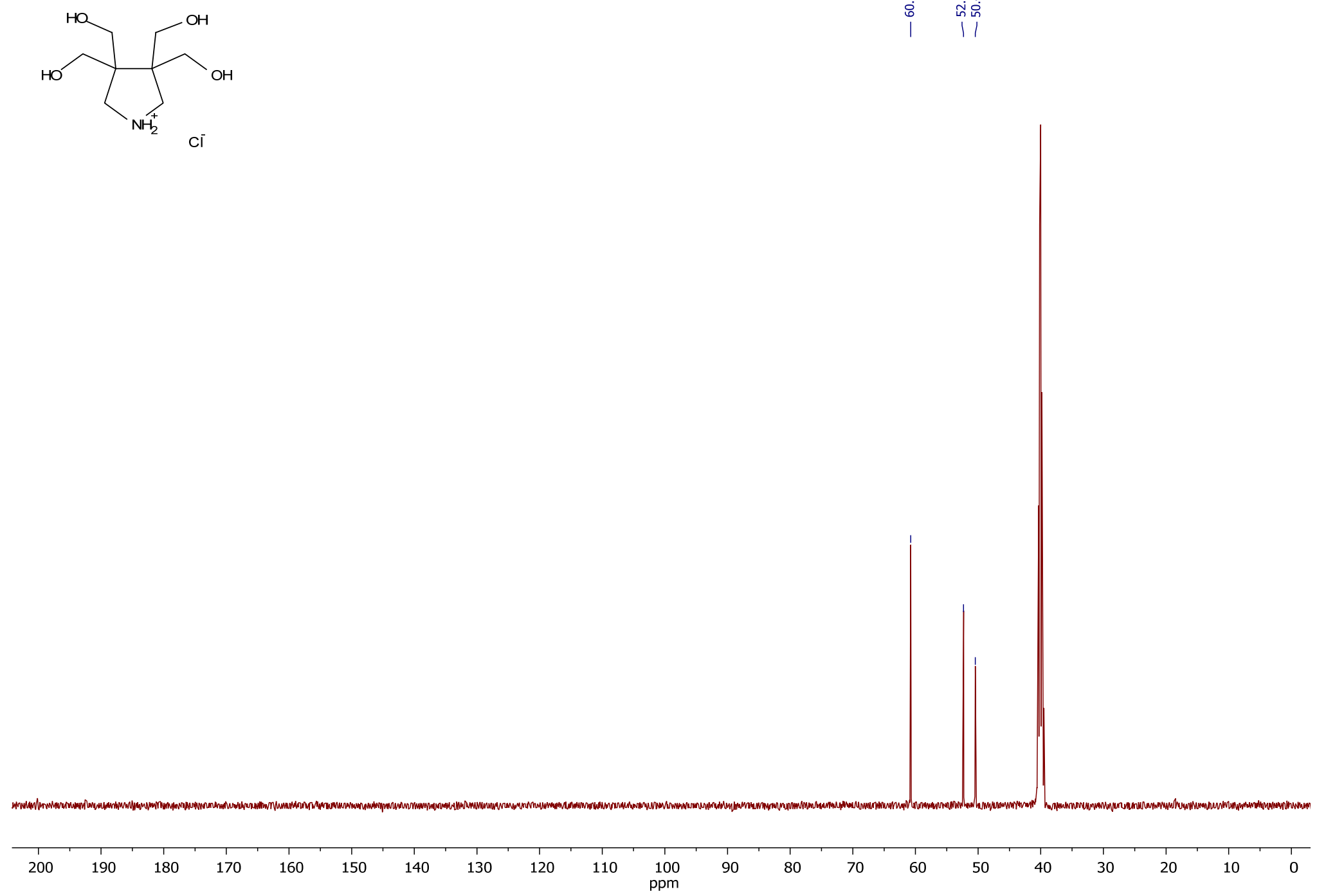

Pyrrolidine-3,3,4,4-tetrayltetramethanol hydrochloride (54) ${ }^{13} \mathrm{C}$ NMR 


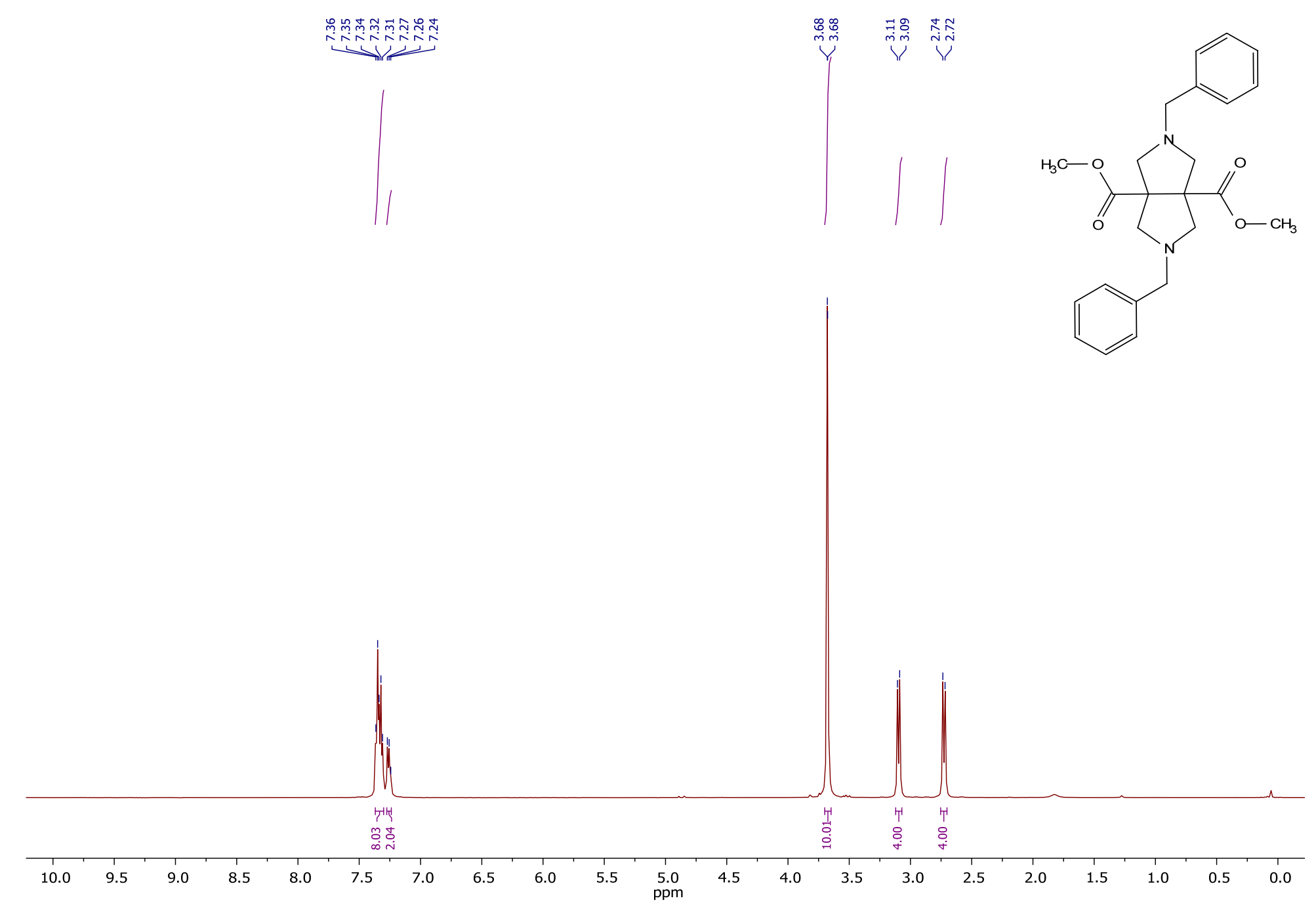

Dimethyl 2,5-dibenzyloctahydropyrrolo[3,4-c]pyrrole-3a,6a-dicarboxylate (56) ${ }^{1} \mathrm{H}$ NMR 


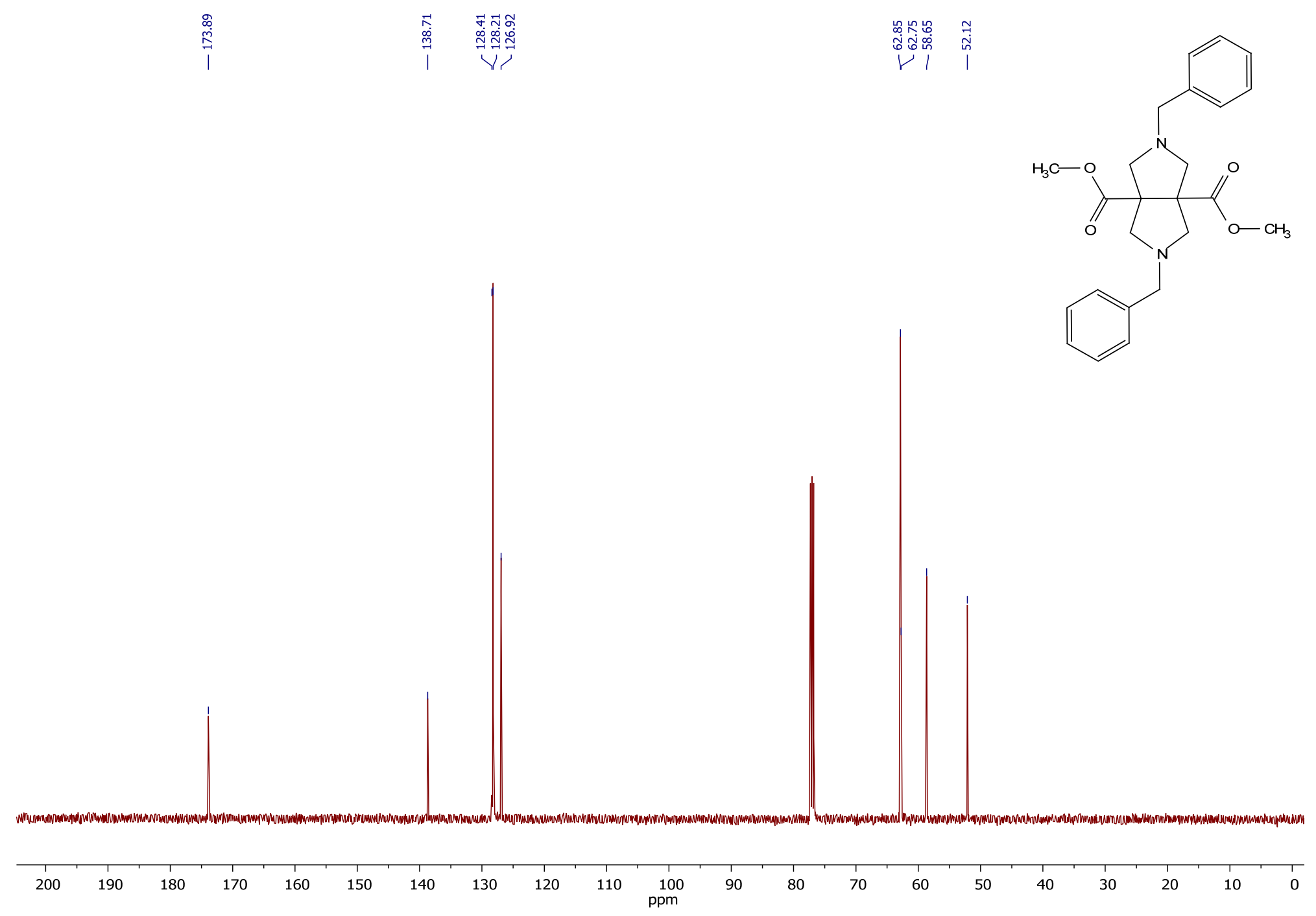

Dimethyl 2,5-dibenzyloctahydropyrrolo[3,4-c]pyrrole-3a,6a-dicarboxylate (56) ${ }^{13} \mathrm{C}$ NMR 


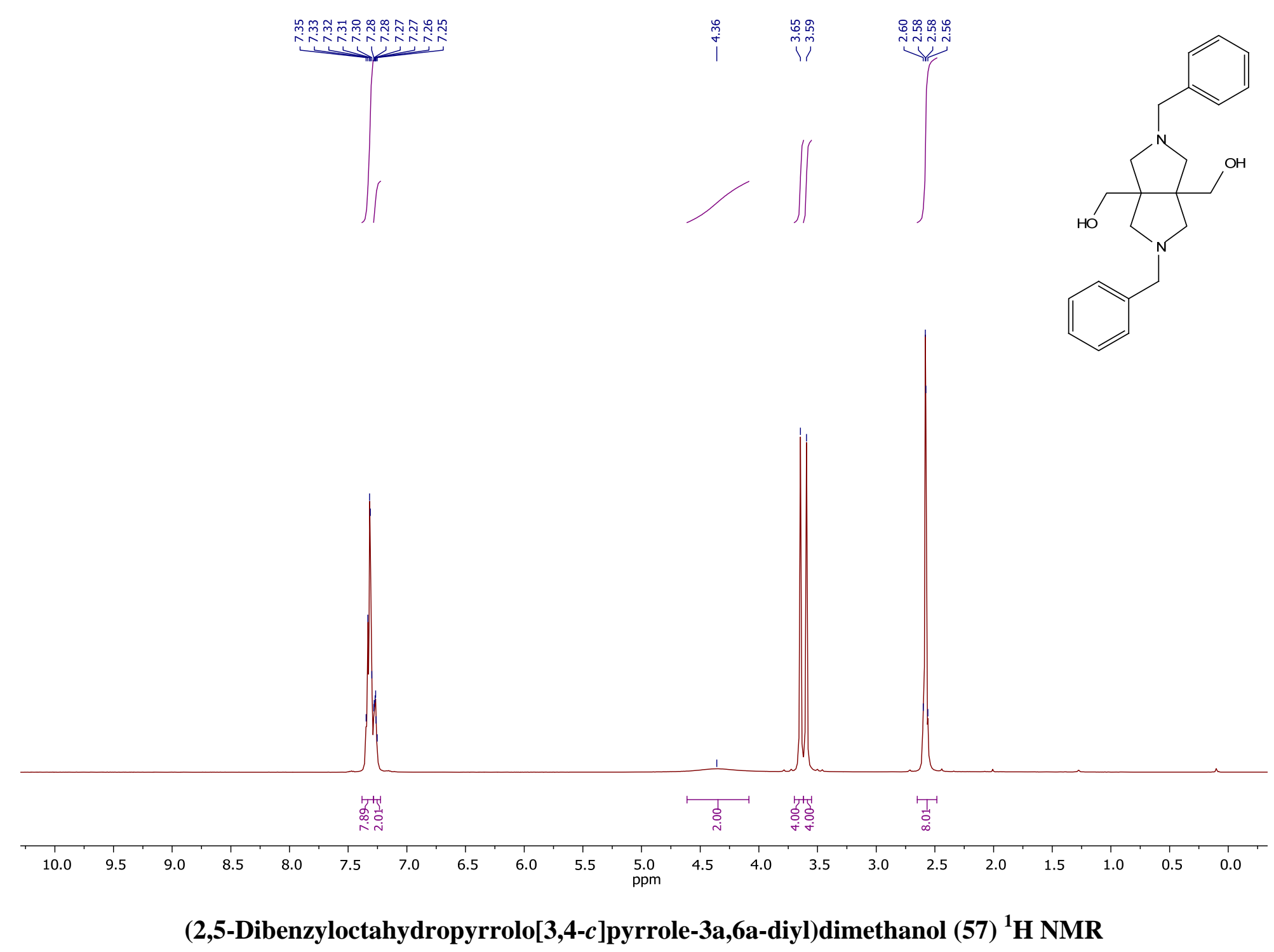




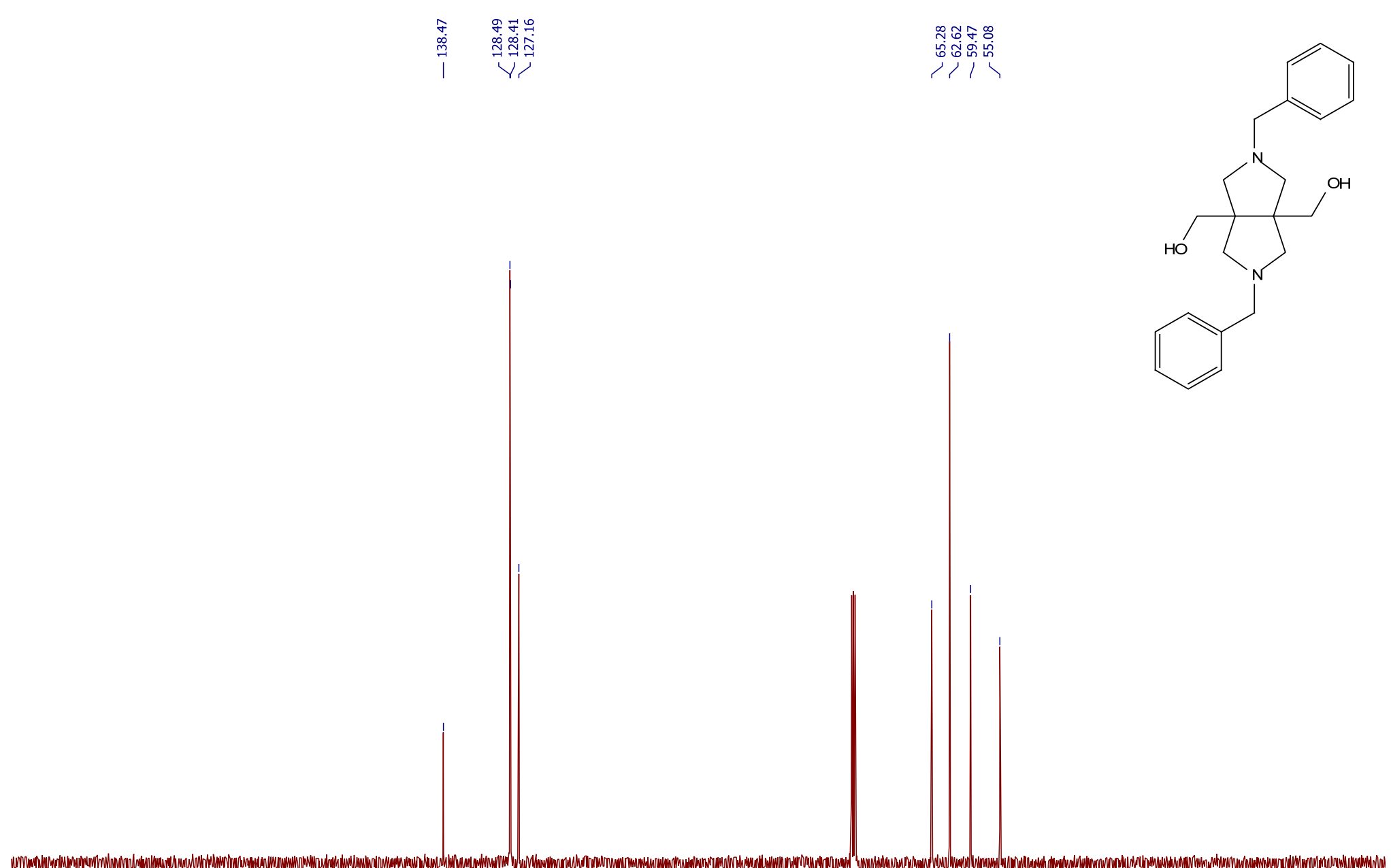

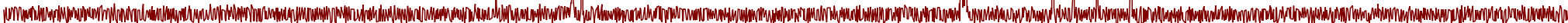

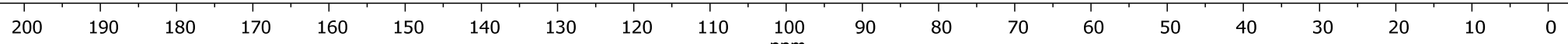

(2,5-Dibenzyloctahydropyrrolo[3,4-c]pyrrole-3a,6a-diyl)dimethanol (57) ${ }^{13} \mathrm{C}$ NMR 


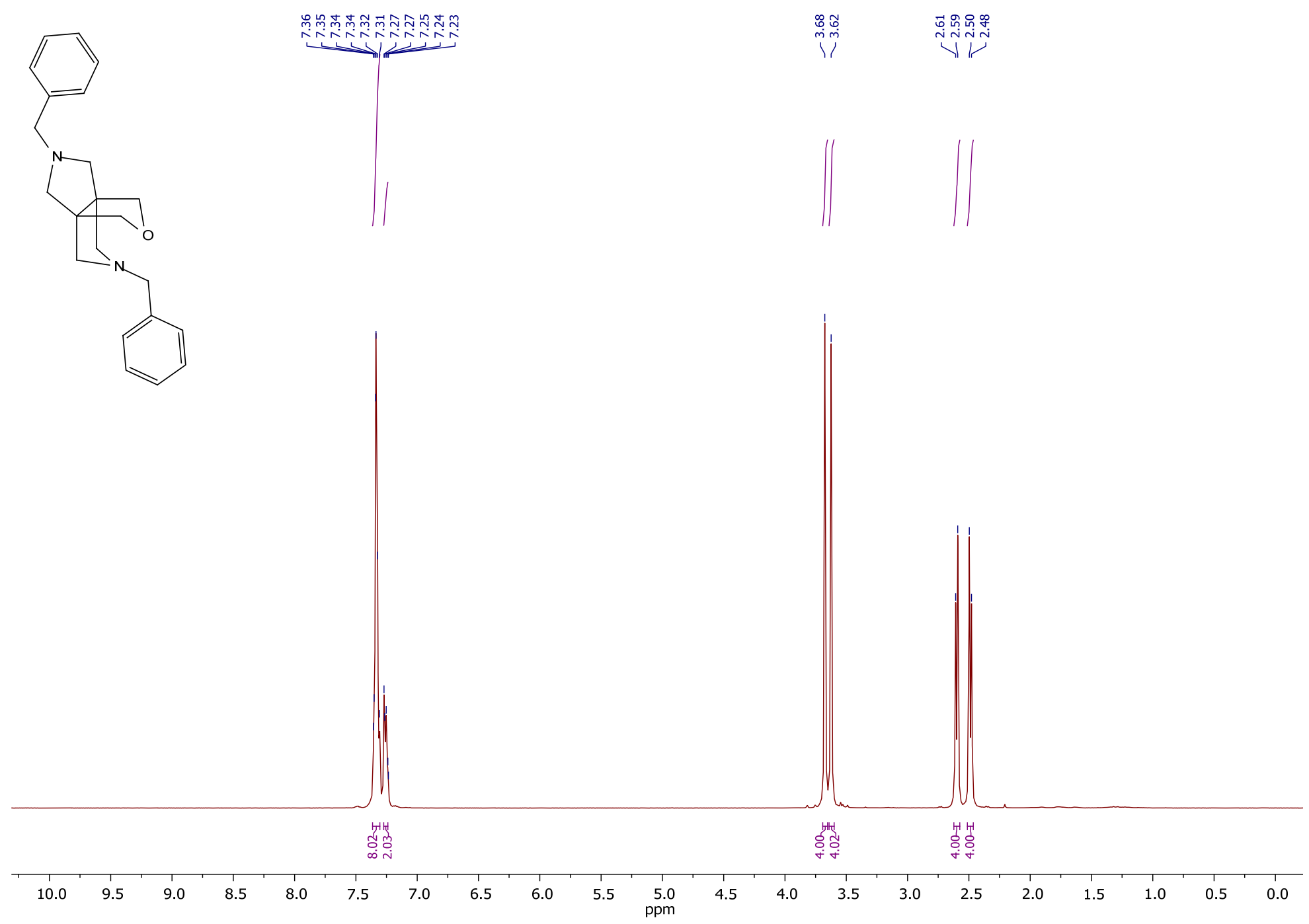

2,5-Dibenzylhexahydro-3a,6a-(methanooxymethano)pyrrolo[3,4-c]pyrrole (58) ${ }^{1} \mathrm{H}$ NMR 

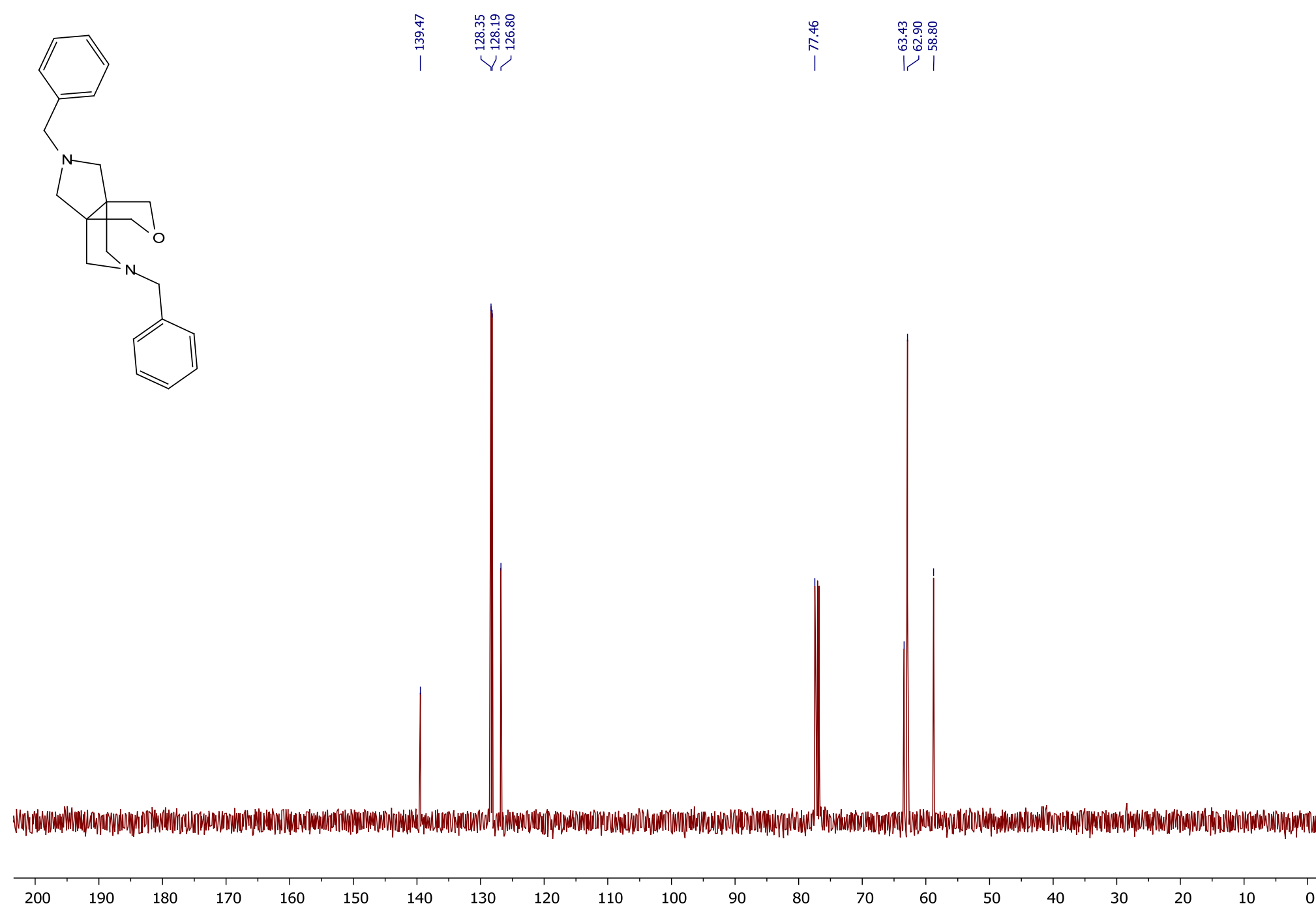

2,5-Dibenzylhexahydro-3a,6a-(methanooxymethano)pyrrolo[3,4-c]pyrrole (58) ${ }^{13} \mathrm{C}$ NMR 


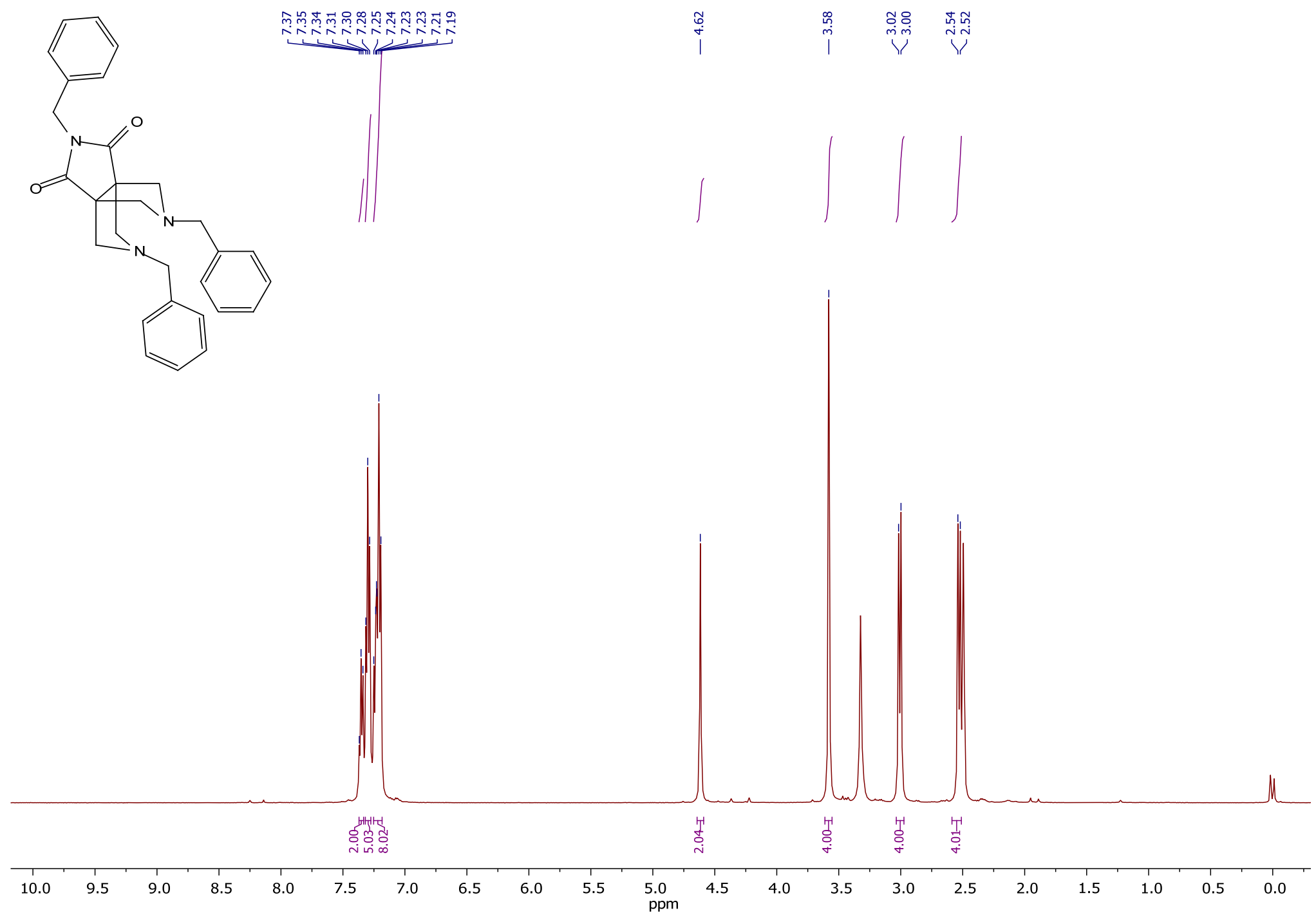

2,5,8-Tribenzyldihydro-3a,6a-(methanoiminomethano)pyrrolo[3,4-c]pyrrole-1,3(2H,4H)-dione (59) ${ }^{1} \mathrm{H}$ NMR 


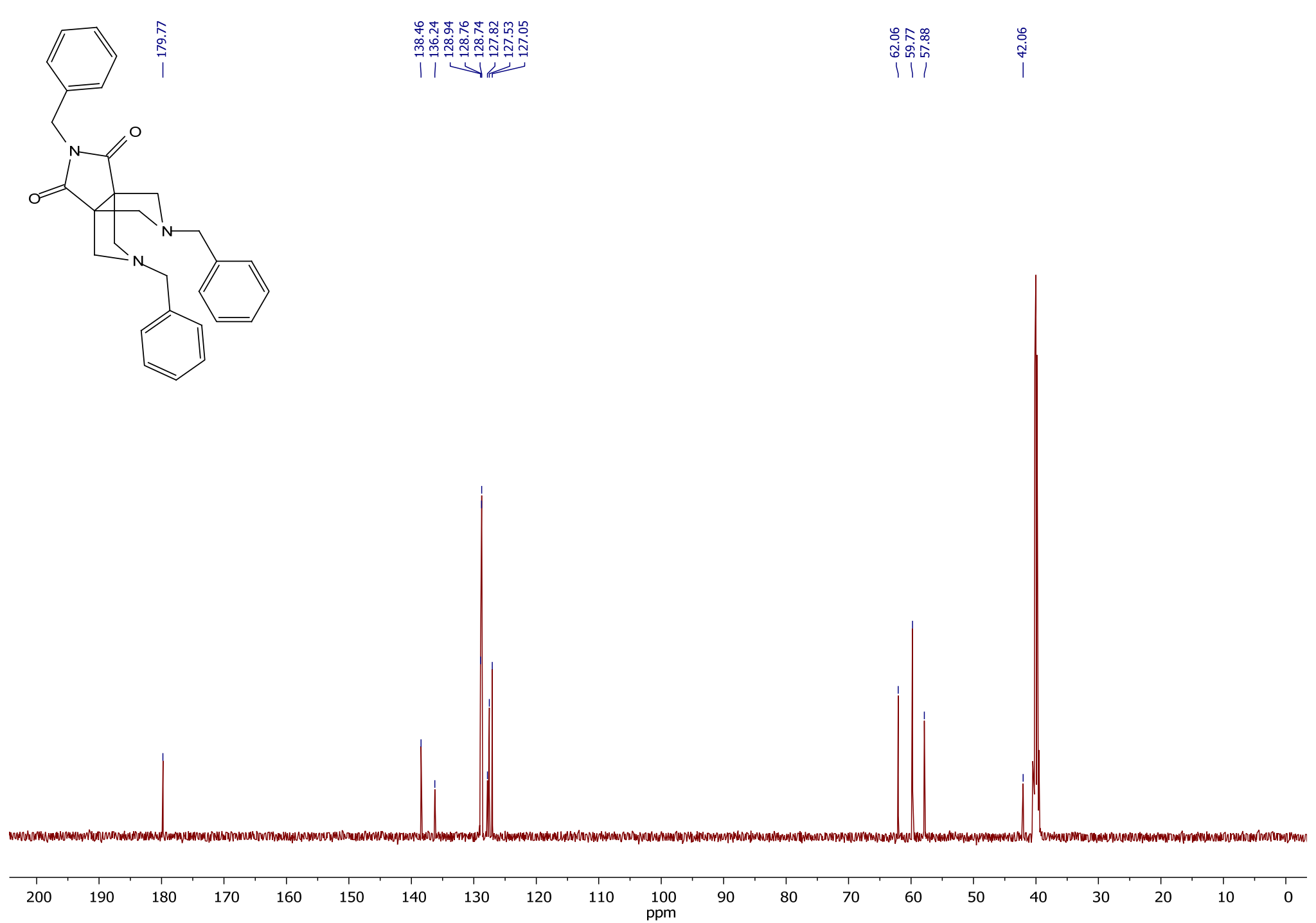

2,5,8-Tribenzyldihydro-3a,6a-(methanoiminomethano)pyrrolo[3,4-c]pyrrole-1,3(2H,4H)-dione (59) ${ }^{13} \mathrm{C}$ NMR 


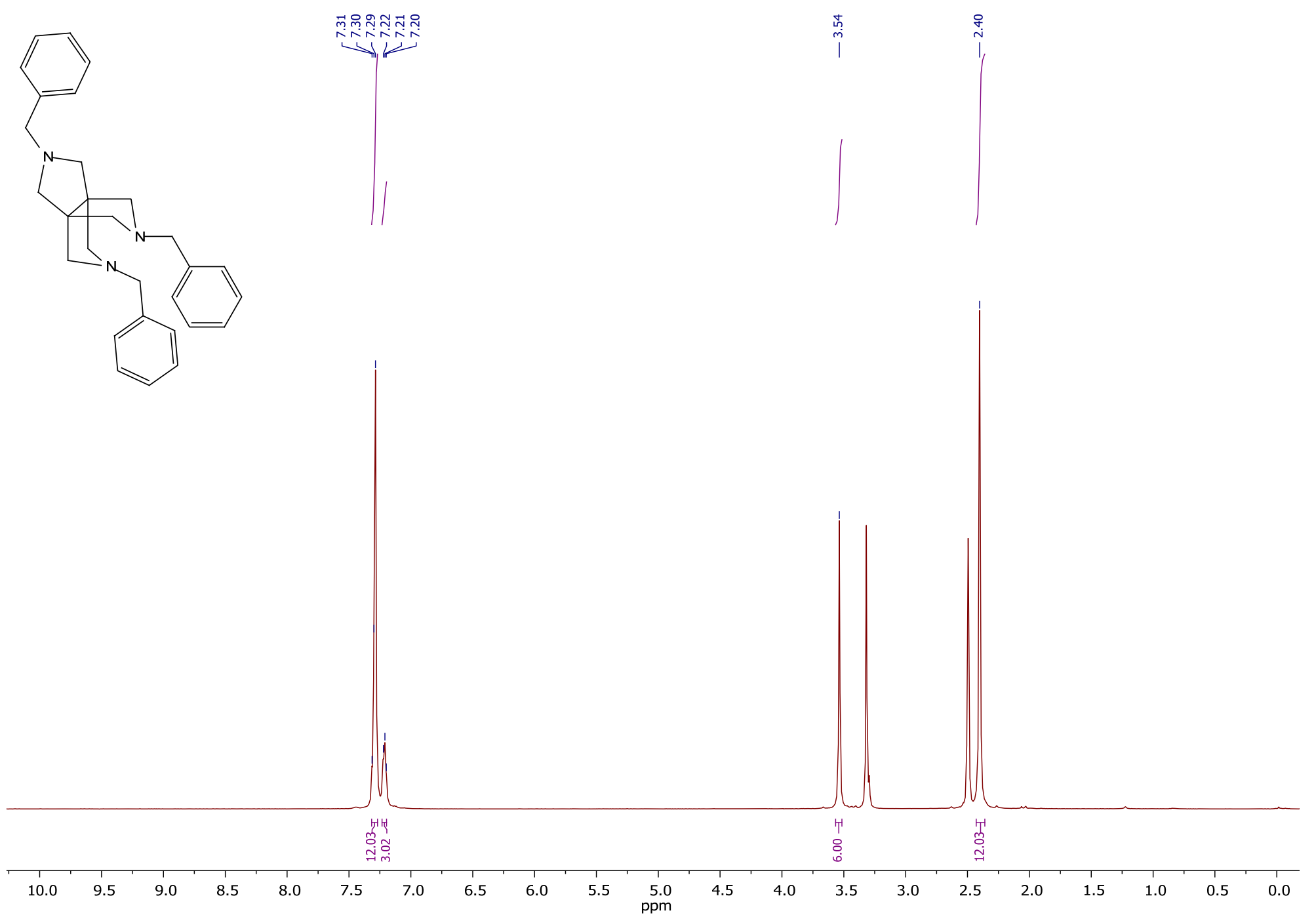

2,5,8-Tribenzylhexahydro-3a,6a-(methanoiminomethano)pyrrolo[3,4-c]pyrrole (60) ${ }^{1} \mathrm{H}$ NMR 


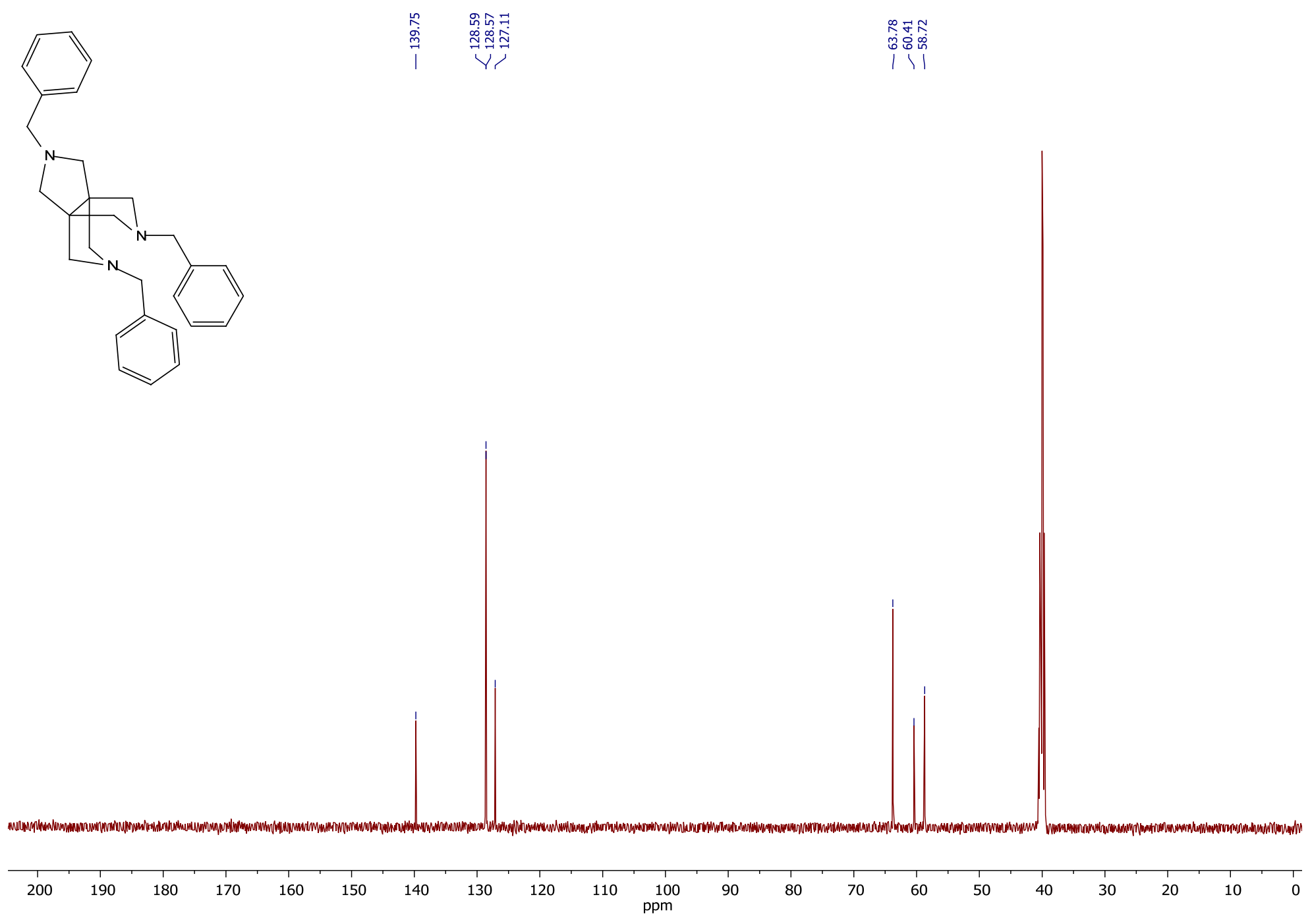

2,5,8-Tribenzylhexahydro-3a,6a-(methanoiminomethano)pyrrolo[3,4-c]pyrrole (60) ${ }^{13} \mathrm{C}$ NMR 


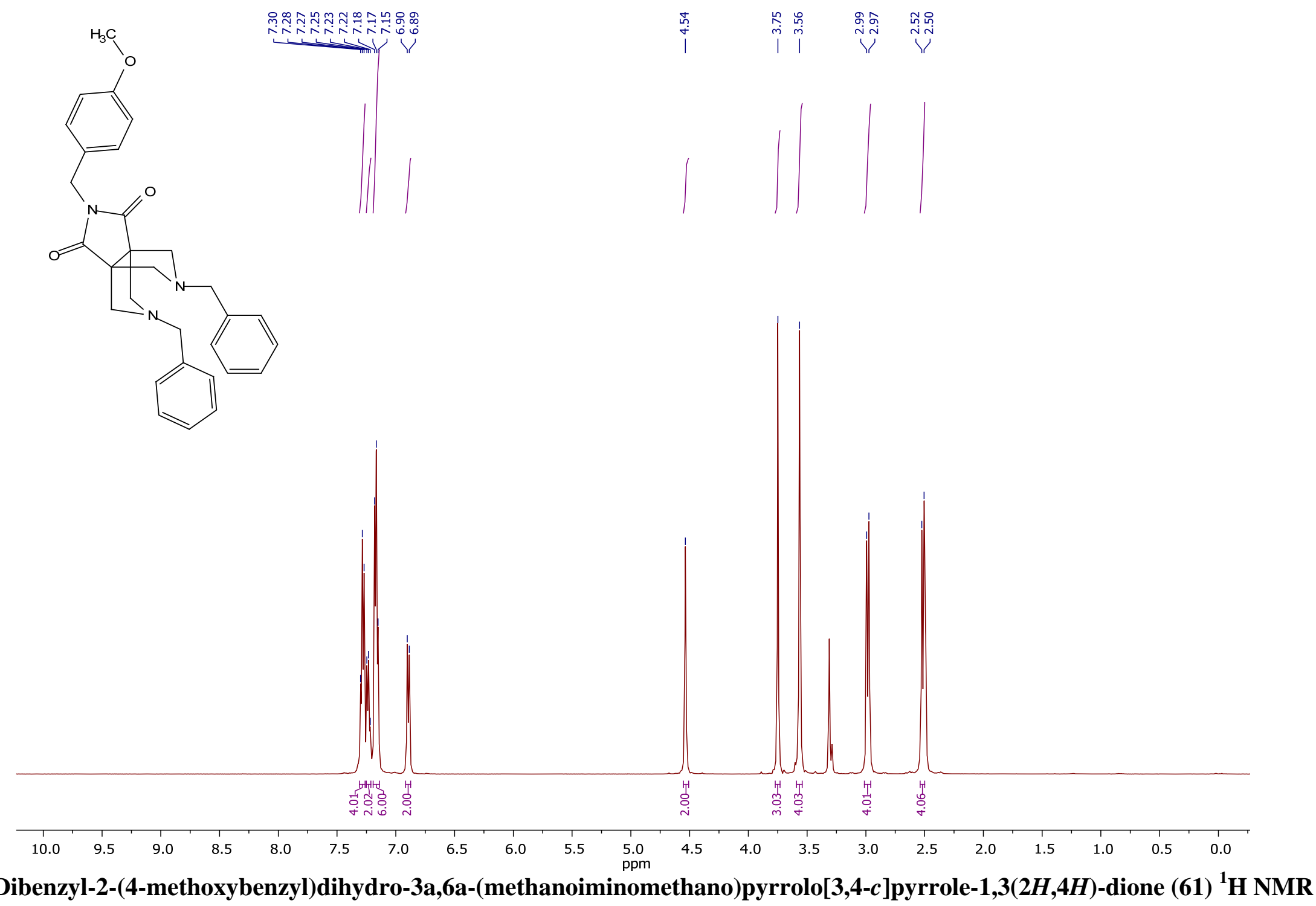




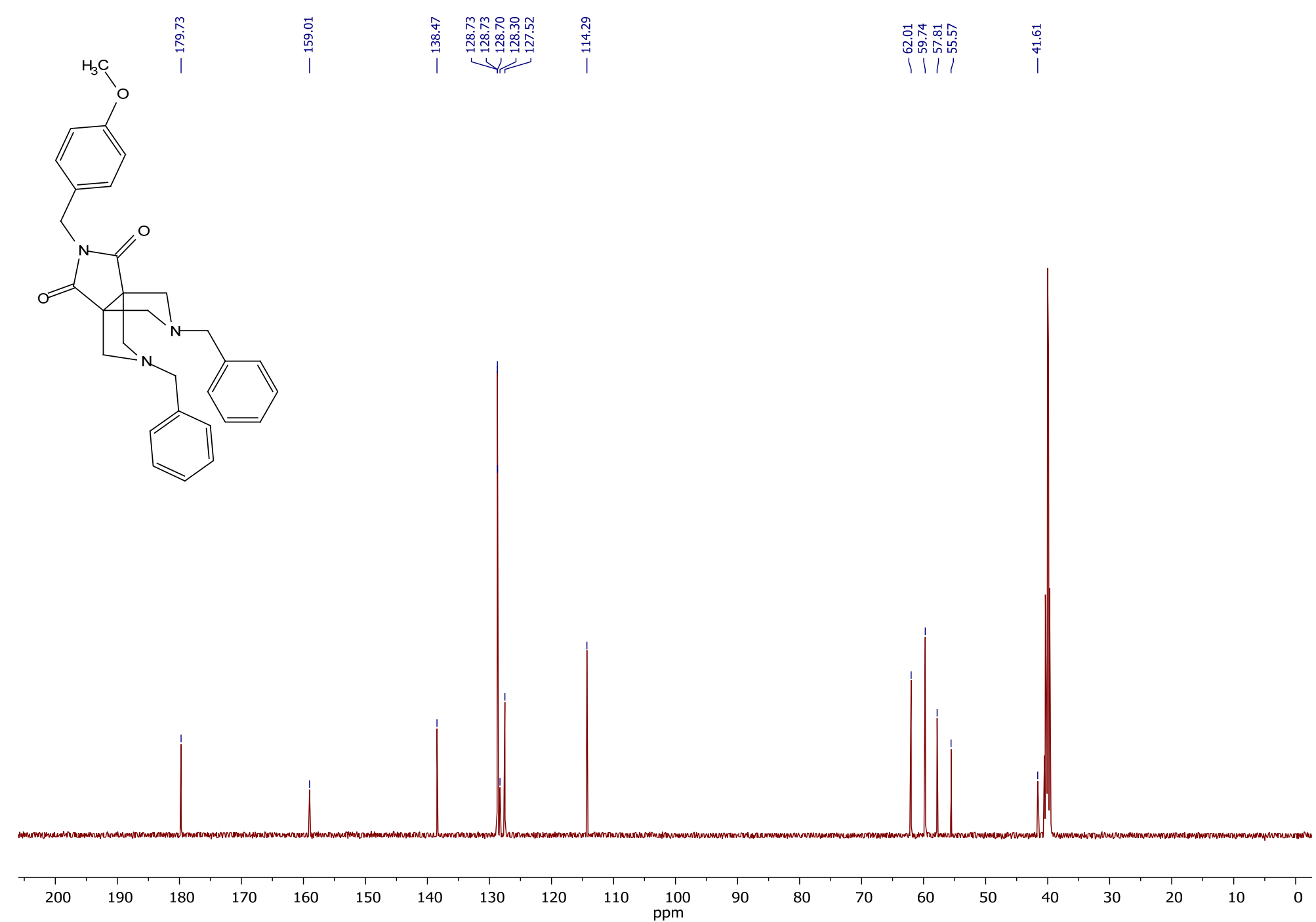

5,8-Dibenzyl-2-(4-methoxybenzyl)dihydro-3a,6a-(methanoiminomethano)pyrrolo[3,4-c]pyrrole-1,3(2H,4H)-dione (61) ${ }^{13} \mathrm{C}$ NMR 


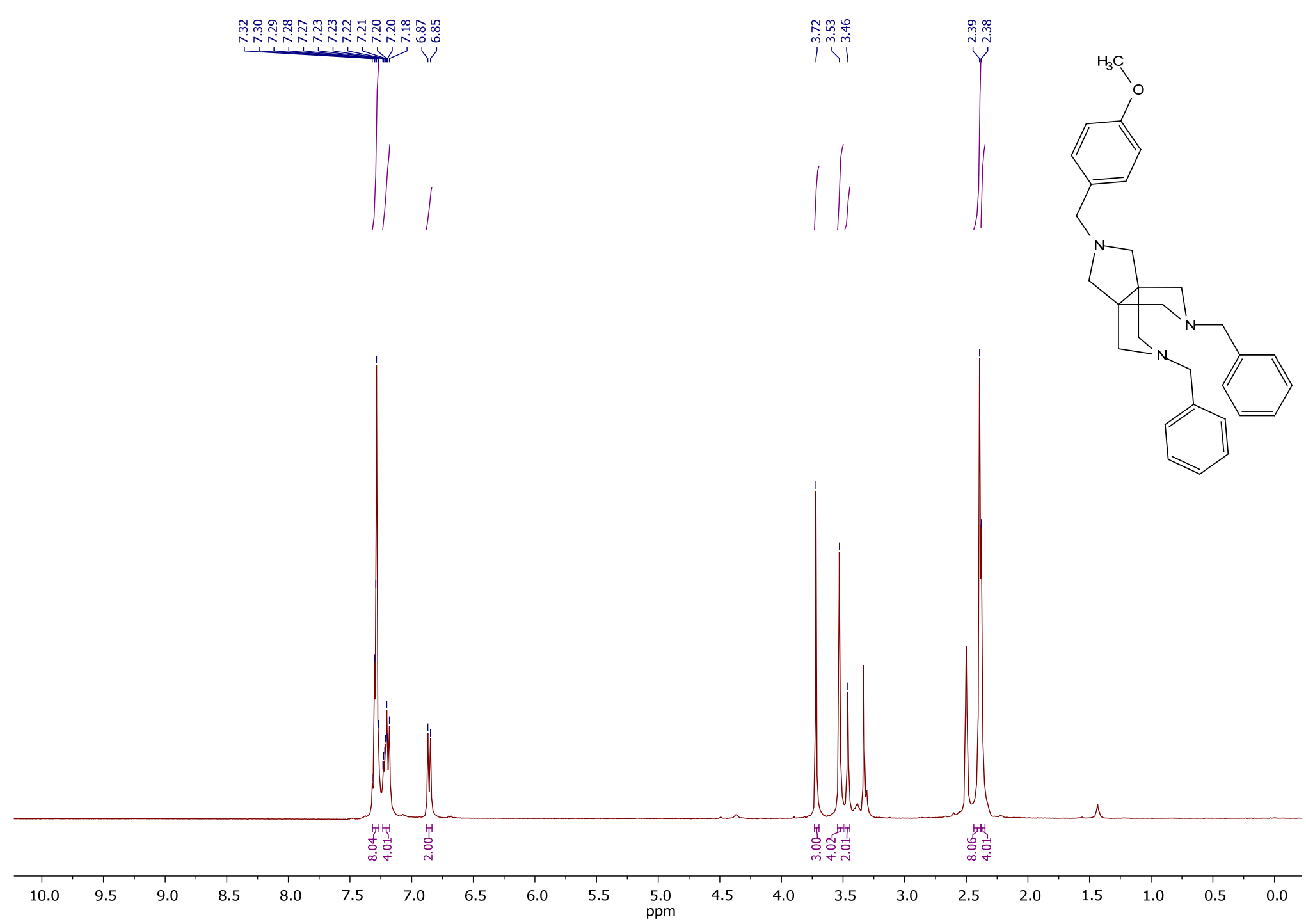

2,5-dibenzyl-8-(4-methoxybenzyl)hexahydro-3a,6a-(methanoiminomethano)pyrrolo[3,4-c]pyrrole (62) ${ }^{1}$ H NMR 


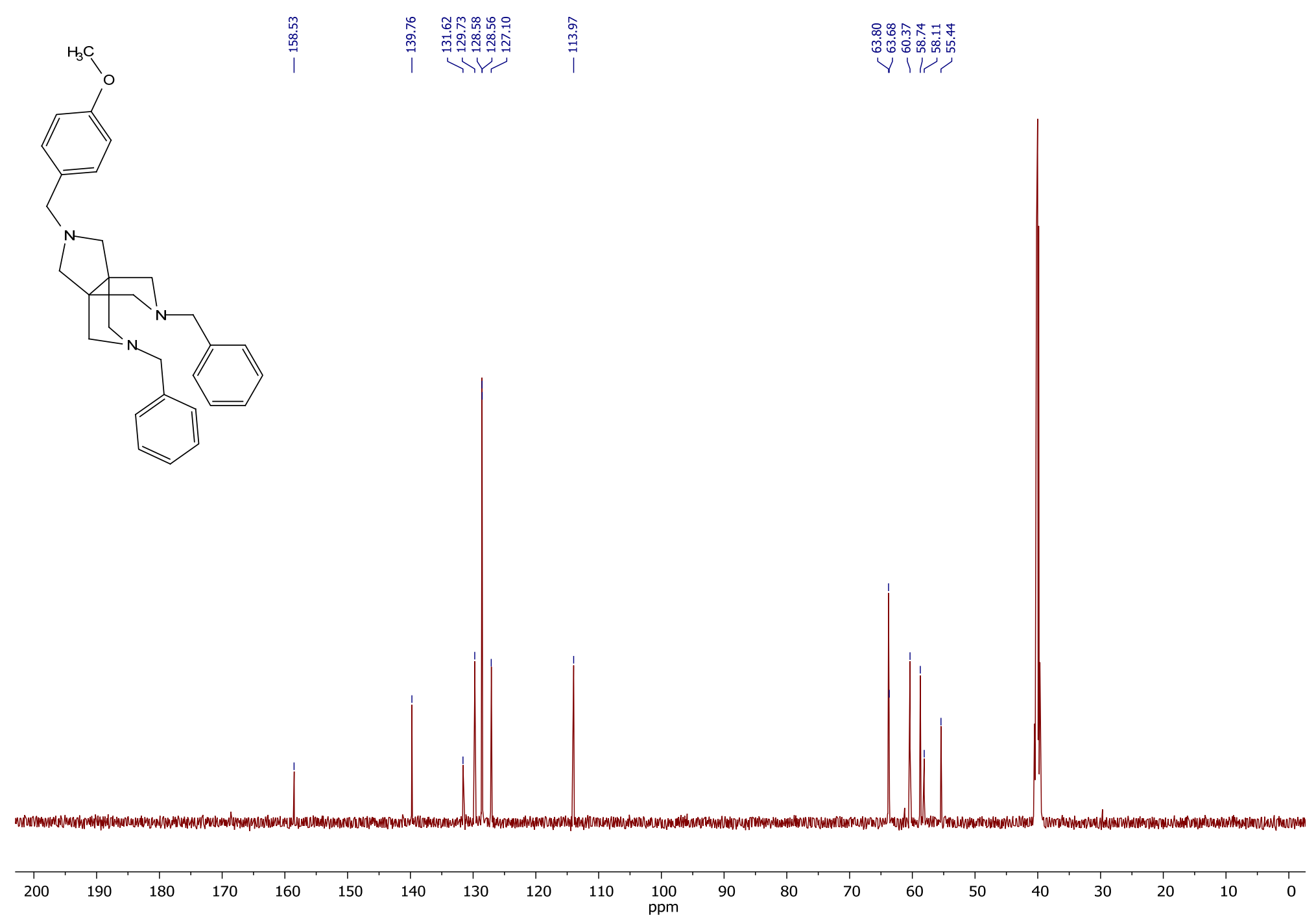

2,5-dibenzyl-8-(4-methoxybenzyl)hexahydro-3a,6a-(methanoiminomethano)pyrrolo[3,4-c]pyrrole (62) ${ }^{13} \mathrm{C}$ NMR 


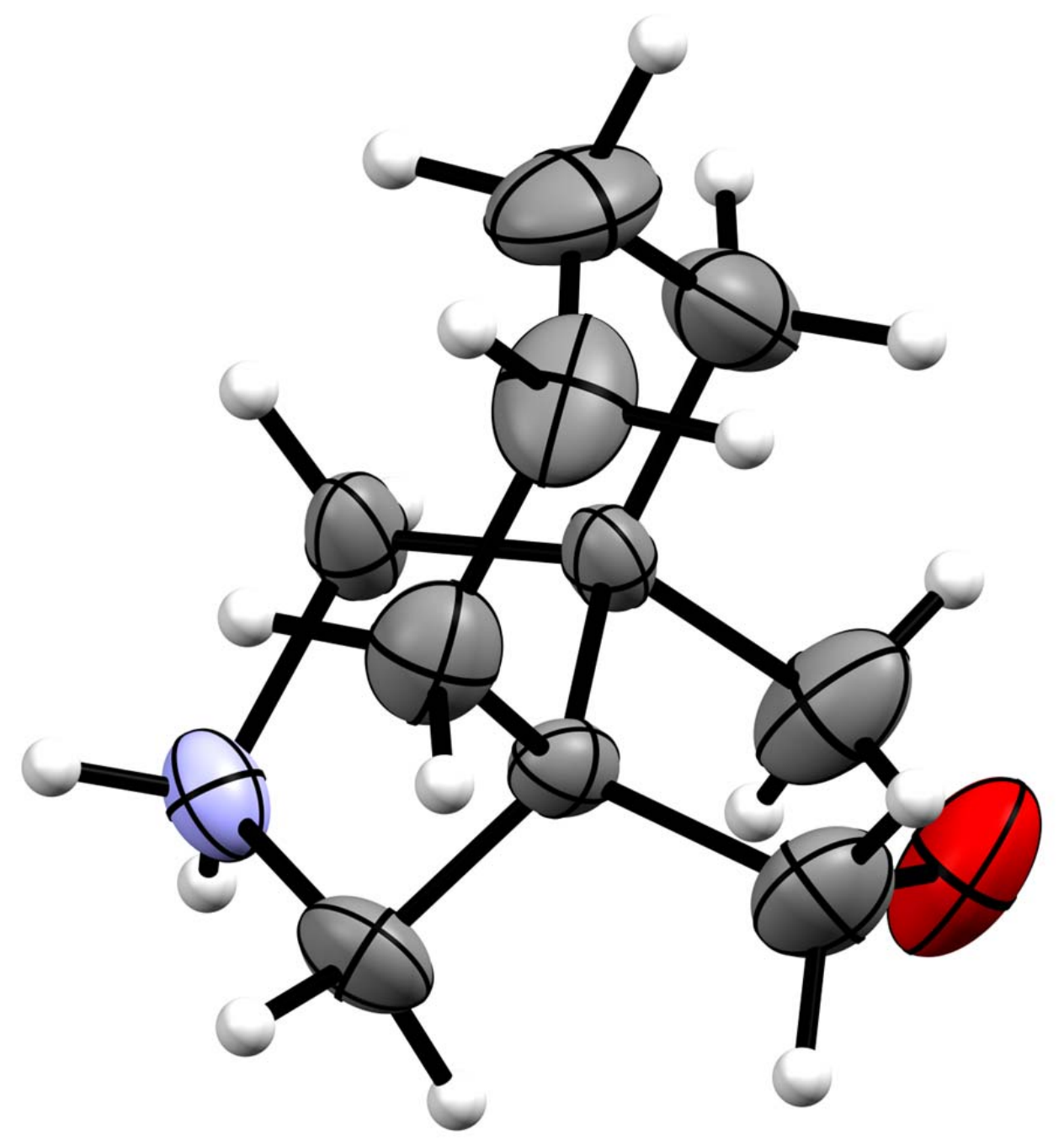

cis-Hexahydro-1H-3a,7a-(methanooxymethano)isoindol-2-ium chloride (14 (A)) ORTEP diagram (Projection 1) (Thermal ellipsoids are shown at 30\% probability level) 


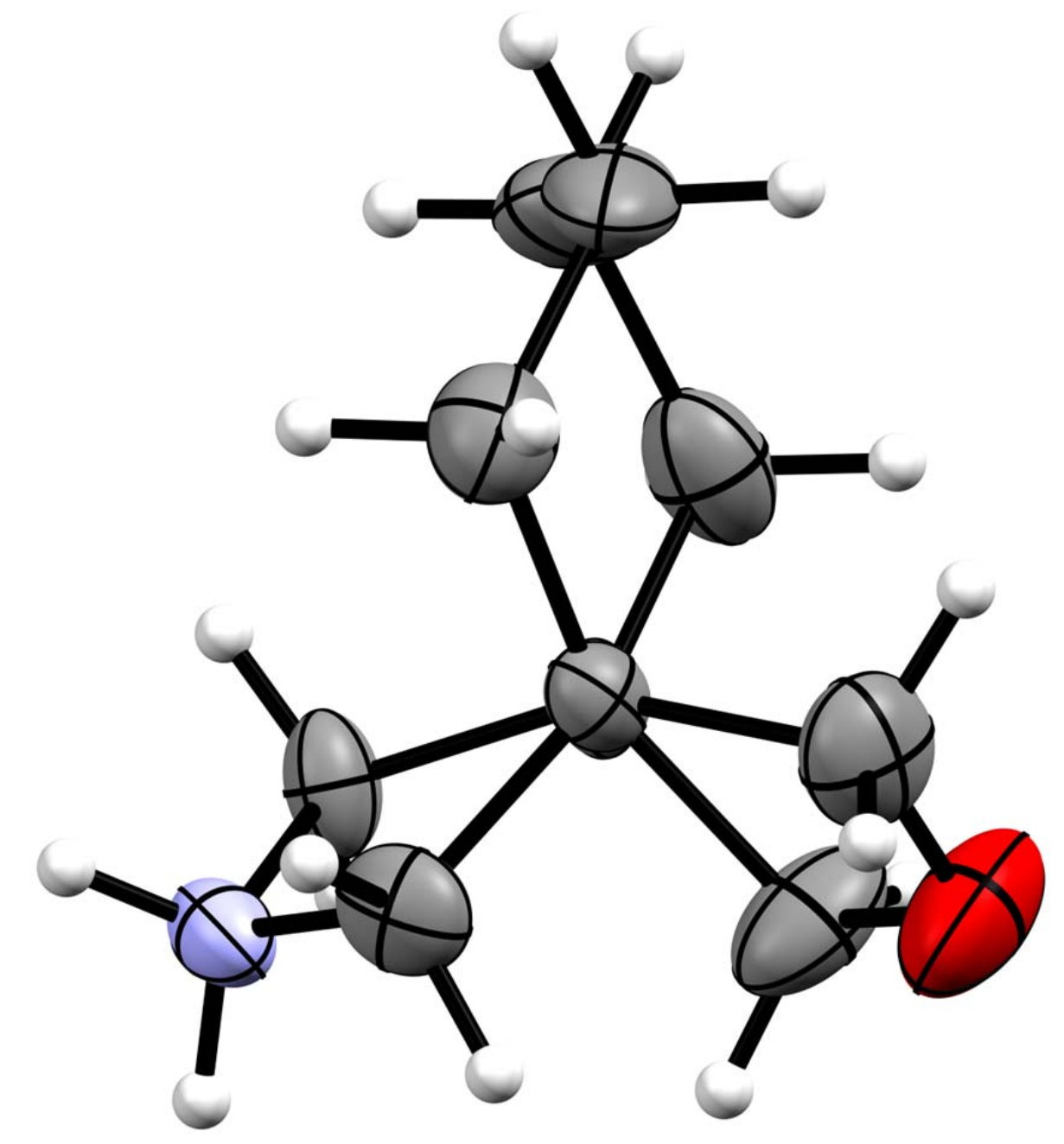

cis-Hexahydro-1H-3a,7a-(methanooxymethano)isoindol-2-ium chloride (14 (A)) ORTEP diagram (Projection 2)

(Thermal ellipsoids are shown at 30\% probability level) 


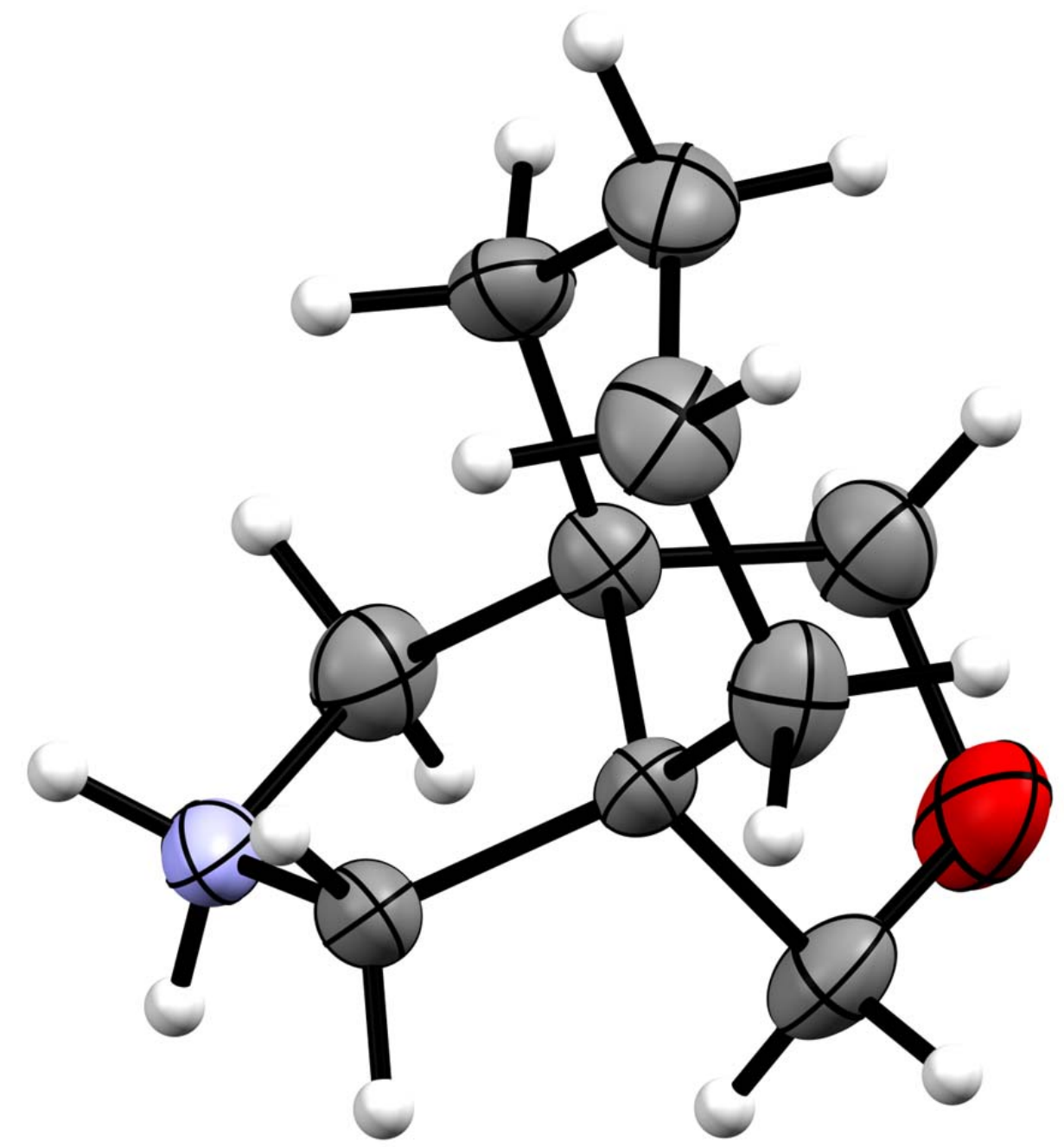

cis-Hexahydro-1H-3a,7a-(methanooxymethano)isoindol-2-ium chloride (14 (B)) ORTEP diagram (Projection 1) (Thermal ellipsoids are shown at $30 \%$ probability level) 


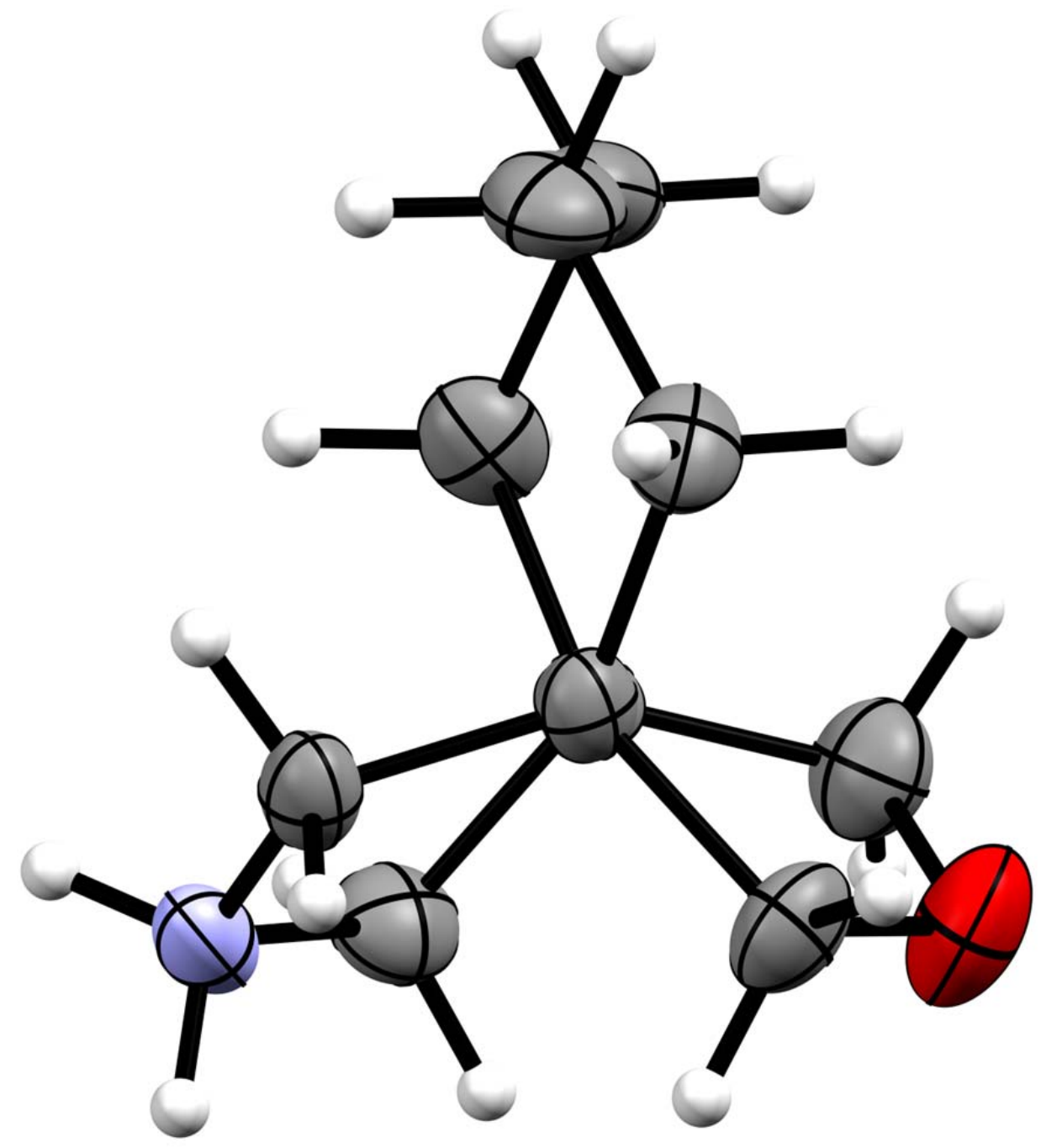

cis-Hexahydro-1H-3a,7a-(methanooxymethano)isoindol-2-ium chloride (14 (B)) ORTEP diagram (Projection 2) (Thermal ellipsoids are shown at $30 \%$ probability level) 


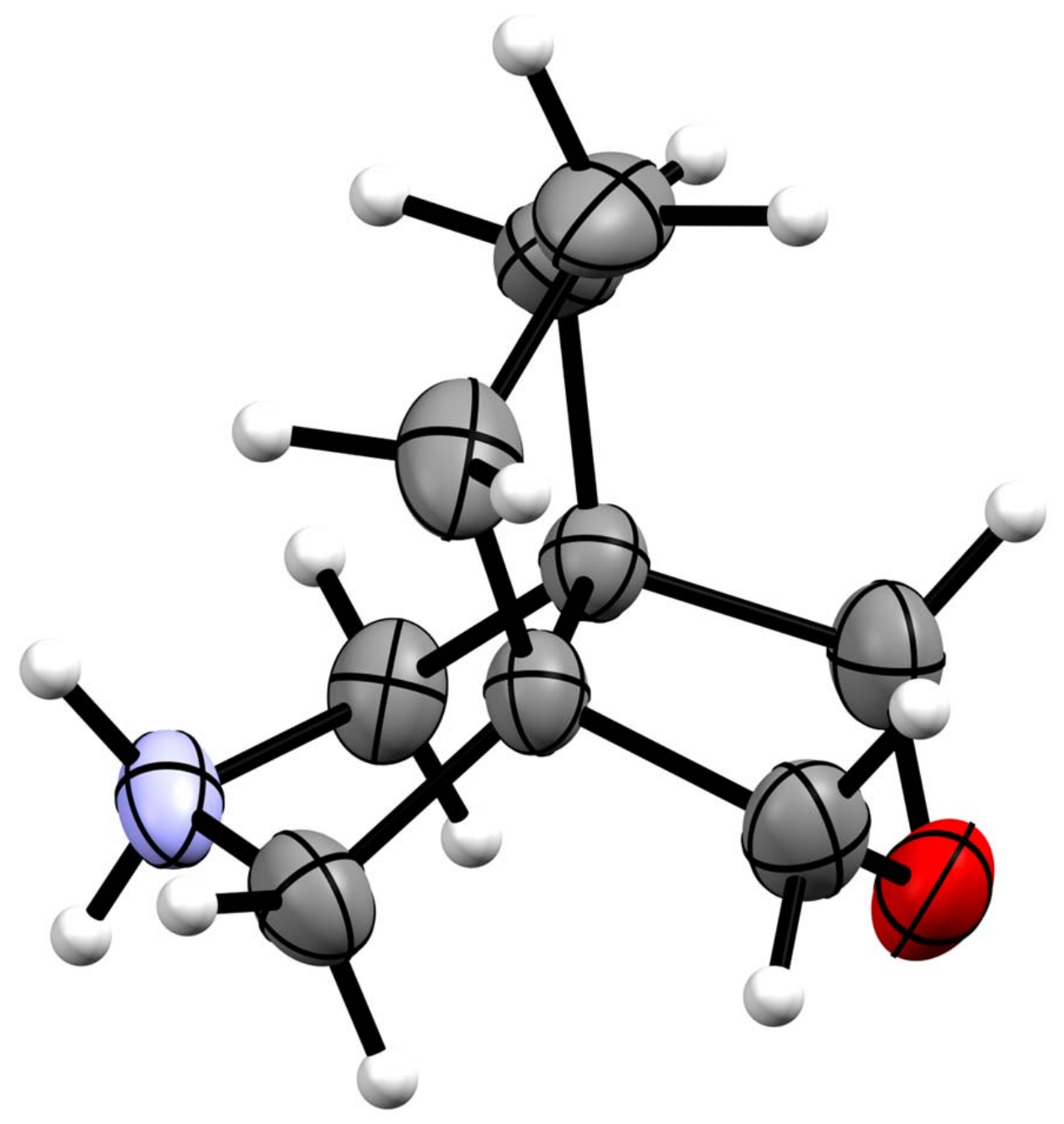

cis-Hexahydro-3a,6a-(methanooxymethano)cyclopenta[c]pyrrol-2-ium chloride (15) ORTEP diagram (Projection 1)

(Thermal ellipsoids are shown at $30 \%$ probability level) 


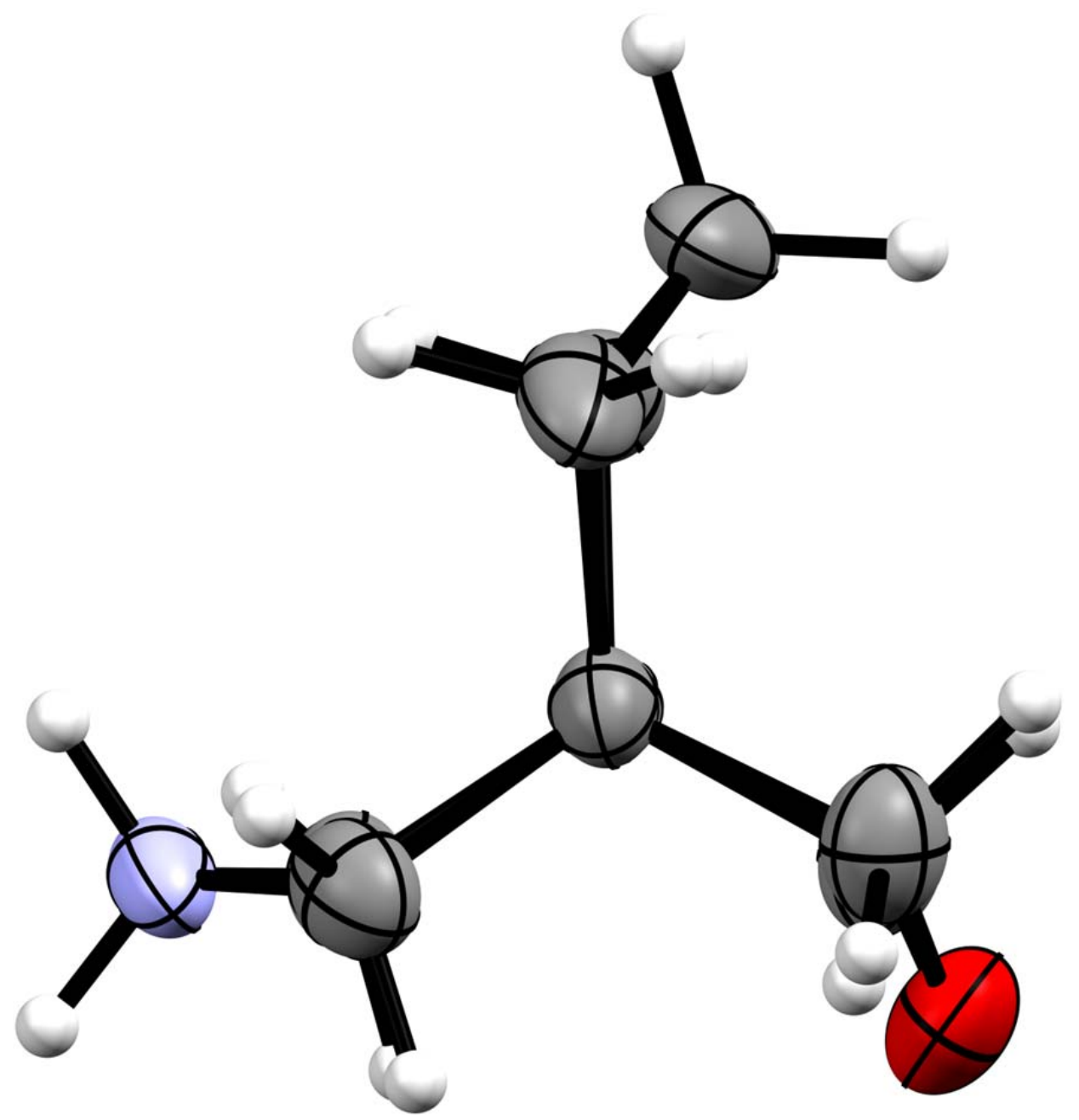

cis-Hexahydro-3a,6a-(methanooxymethano)cyclopenta[c]pyrrol-2-ium chloride (15) ORTEP diagram (Projection 2)

(Thermal ellipsoids are shown at $30 \%$ probability level) 


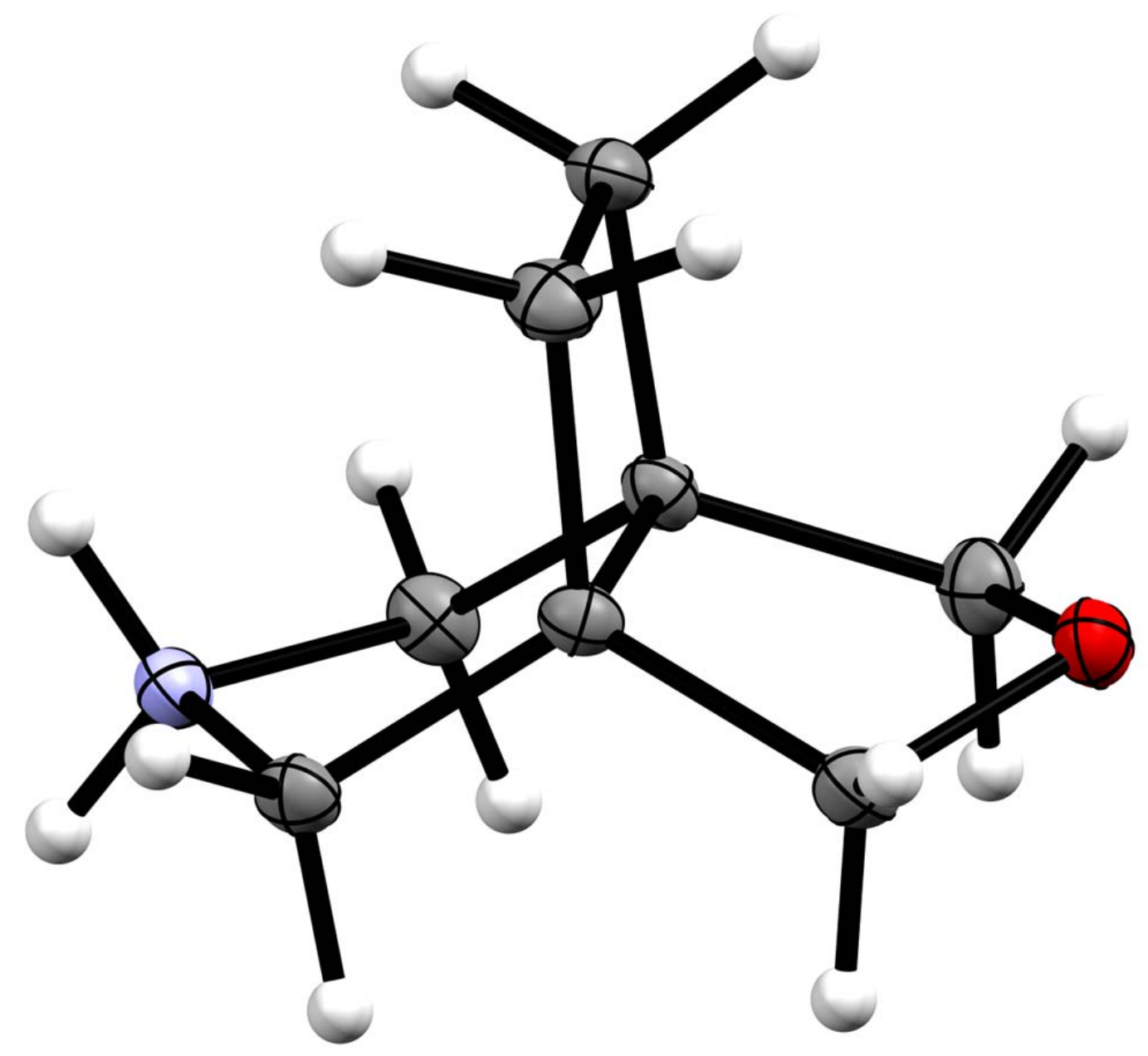

Tetrahydro-1H-3a,6a-ethanofuro[3,4-c]pyrrol-5-ium chloride (16) ORTEP diagram (Projection 1) (Thermal ellipsoids are shown at 30\% probability level) 


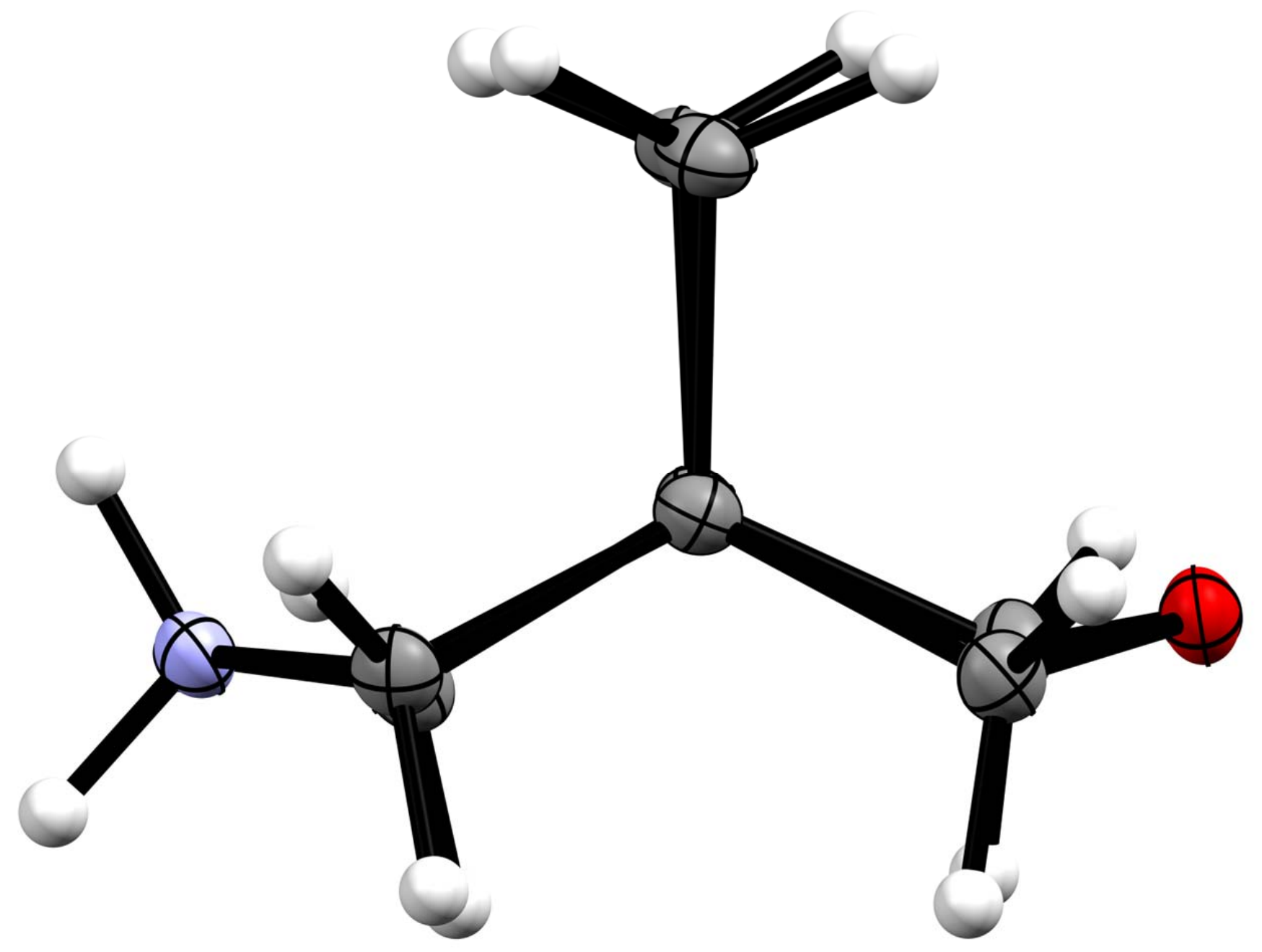

Tetrahydro-1H-3a,6a-ethanofuro[3,4-c]pyrrol-5-ium chloride (16) ORTEP diagram (Projection 2)

(Thermal ellipsoids are shown at $30 \%$ probability level) 


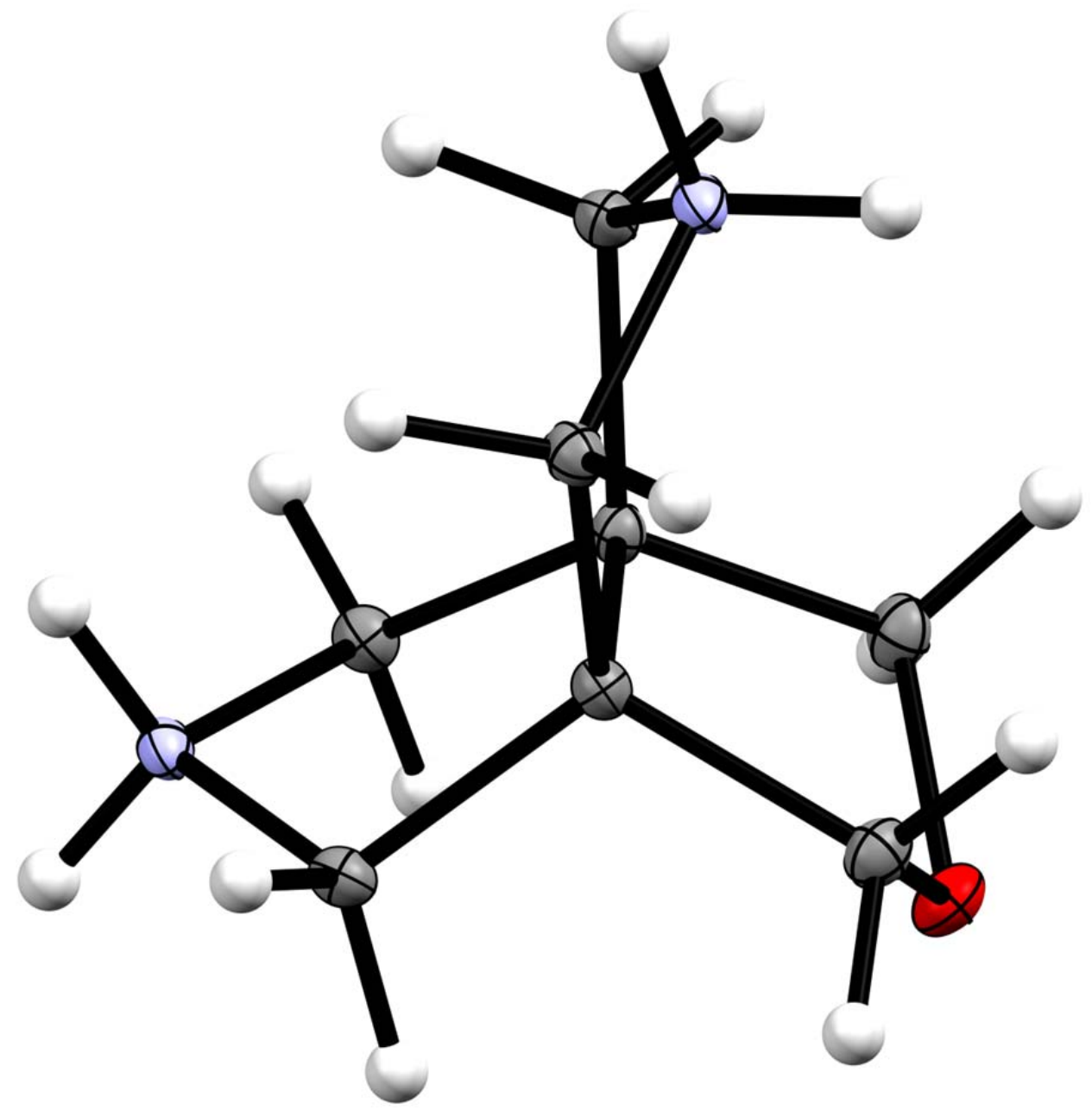

Hexahydro-3a,6a-(methanooxymethano)pyrrolo[3,4-c]pyrrole-2,5-diium chloride (18 (A)) ORTEP diagram (Projection 1) (Thermal ellipsoids are shown at $30 \%$ probability level) 


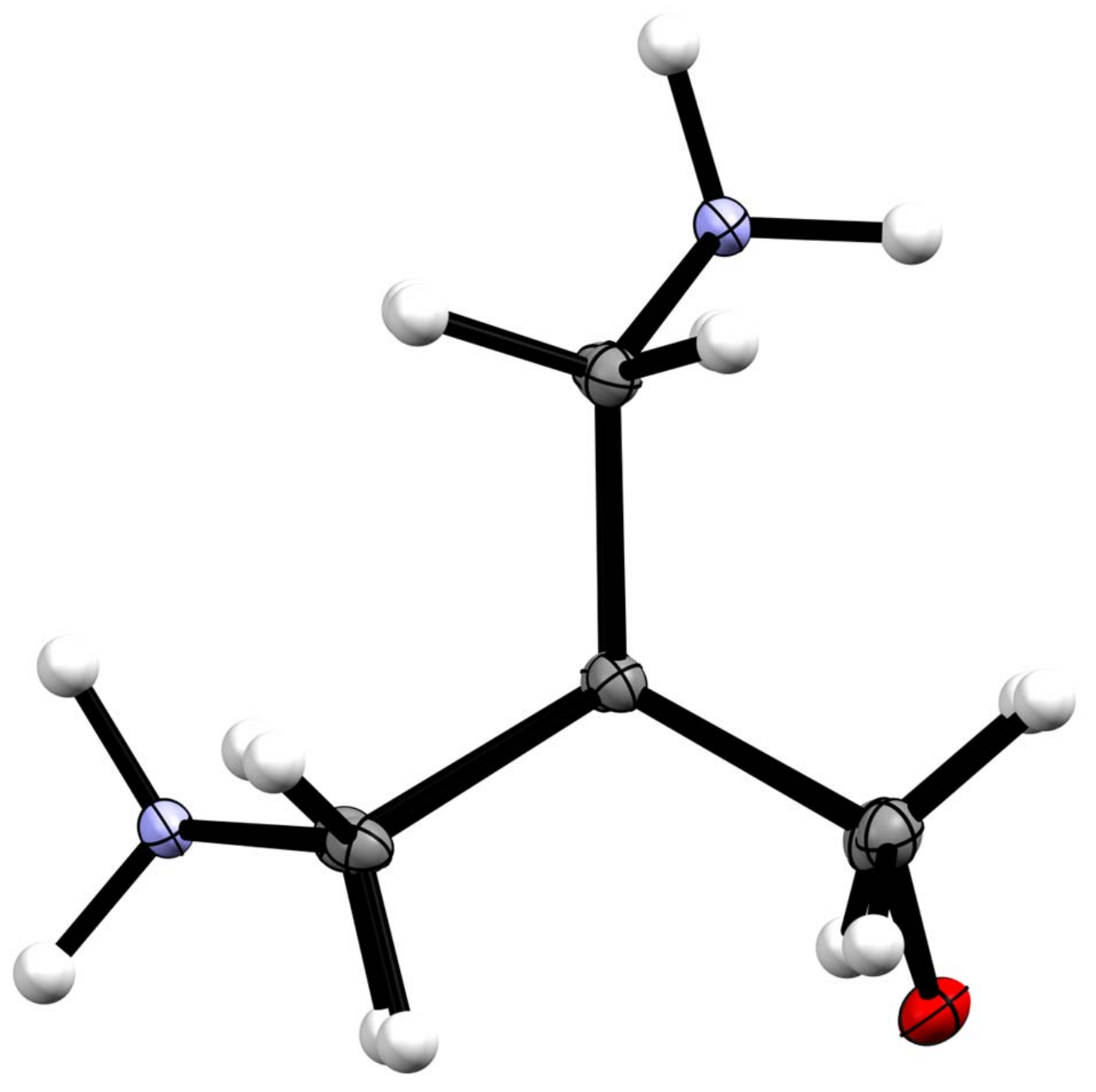

Hexahydro-3a,6a-(methanooxymethano)pyrrolo[3,4-c]pyrrole-2,5-diium chloride (18 (A)) ORTEP diagram (Projection 2) (Thermal ellipsoids are shown at $30 \%$ probability level) 


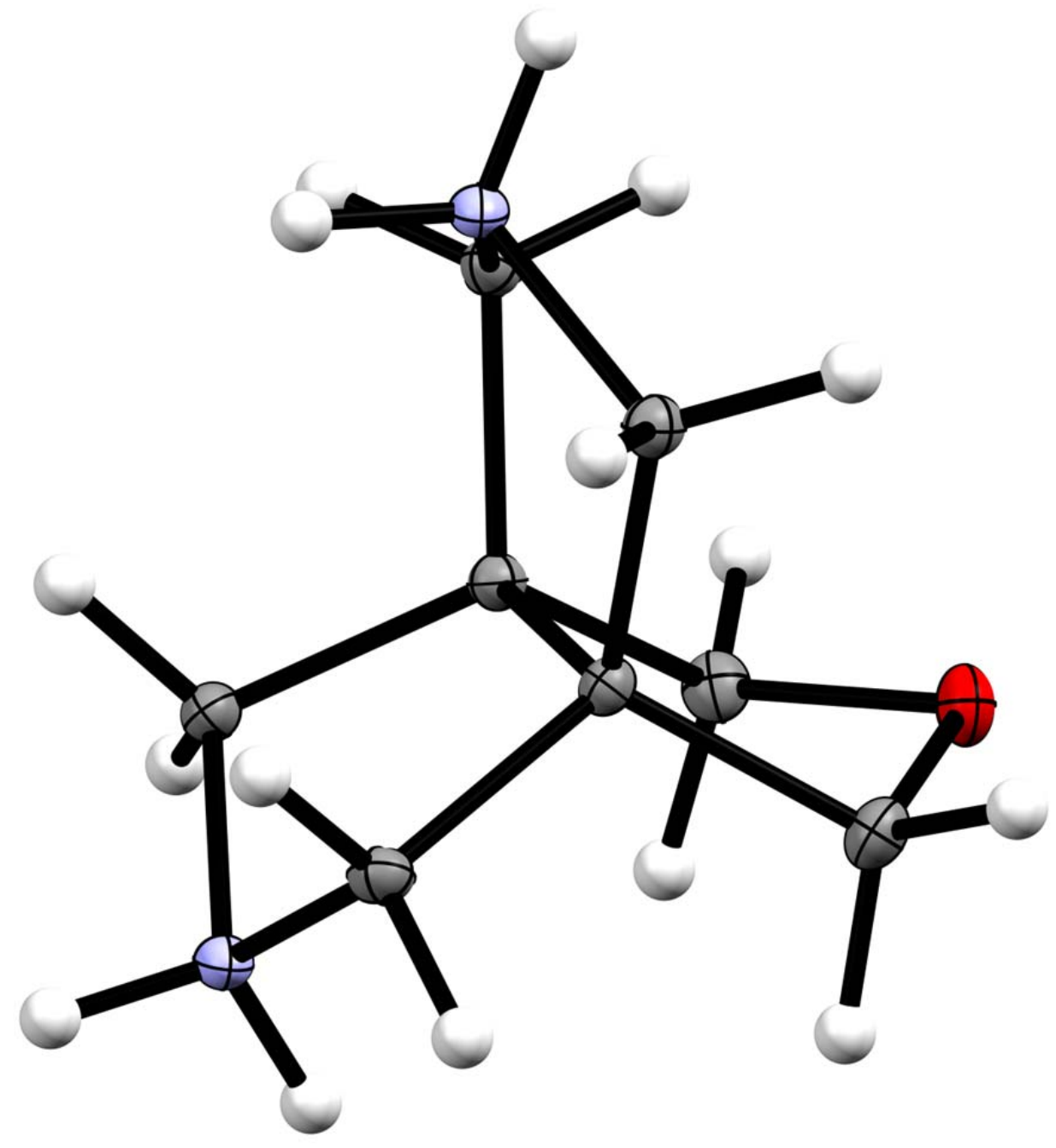

Hexahydro-3a,6a-(methanooxymethano)pyrrolo[3,4-c]pyrrole-2,5-diium chloride (18 (B)) ORTEP diagram (Projection 1) (Thermal ellipsoids are shown at 30\% probability level) 


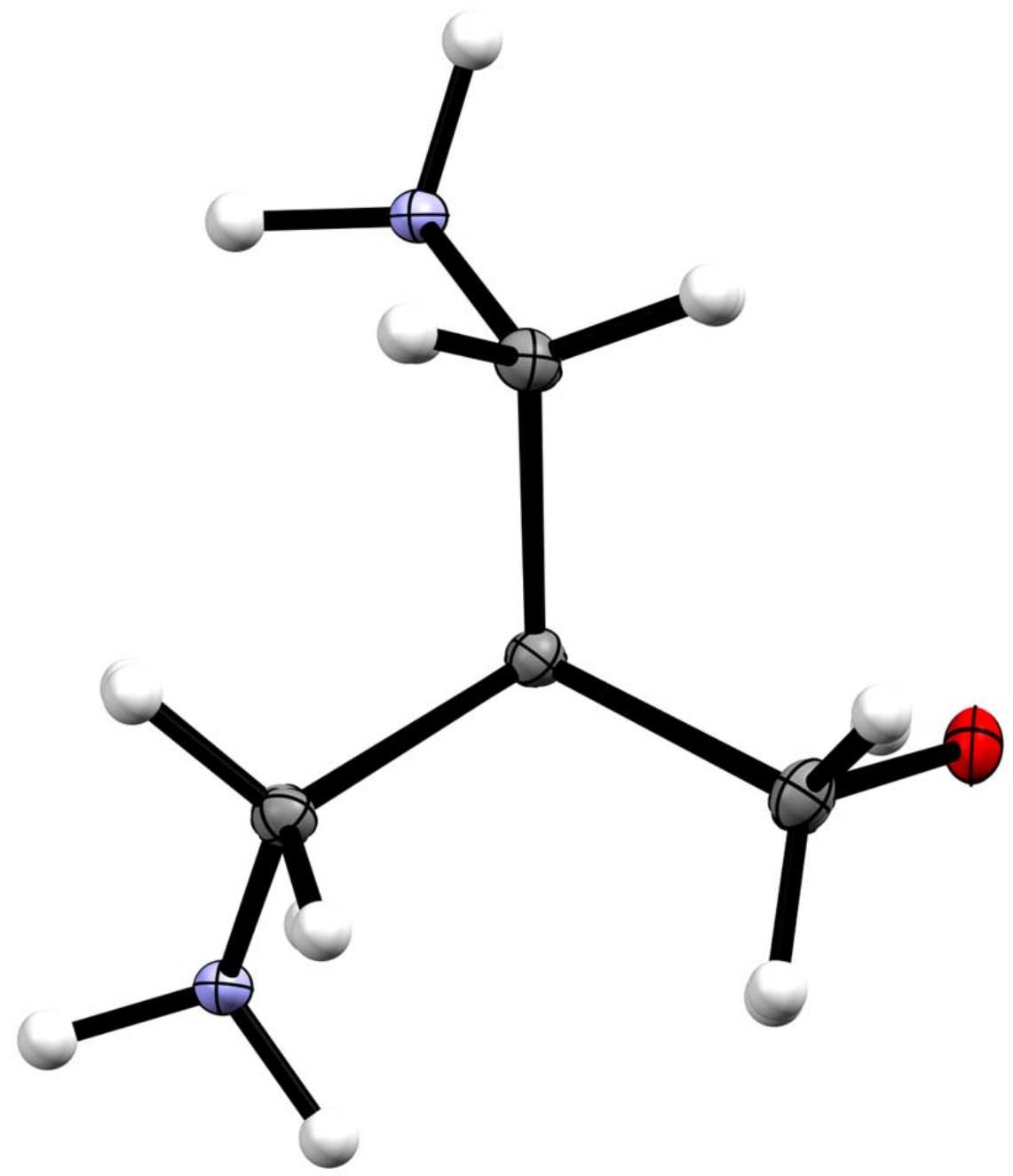

Hexahydro-3a,6a-(methanooxymethano)pyrrolo[3,4-c]pyrrole-2,5-diium chloride (18 (B)) ORTEP diagram (Projection 2) (Thermal ellipsoids are shown at $30 \%$ probability level) 


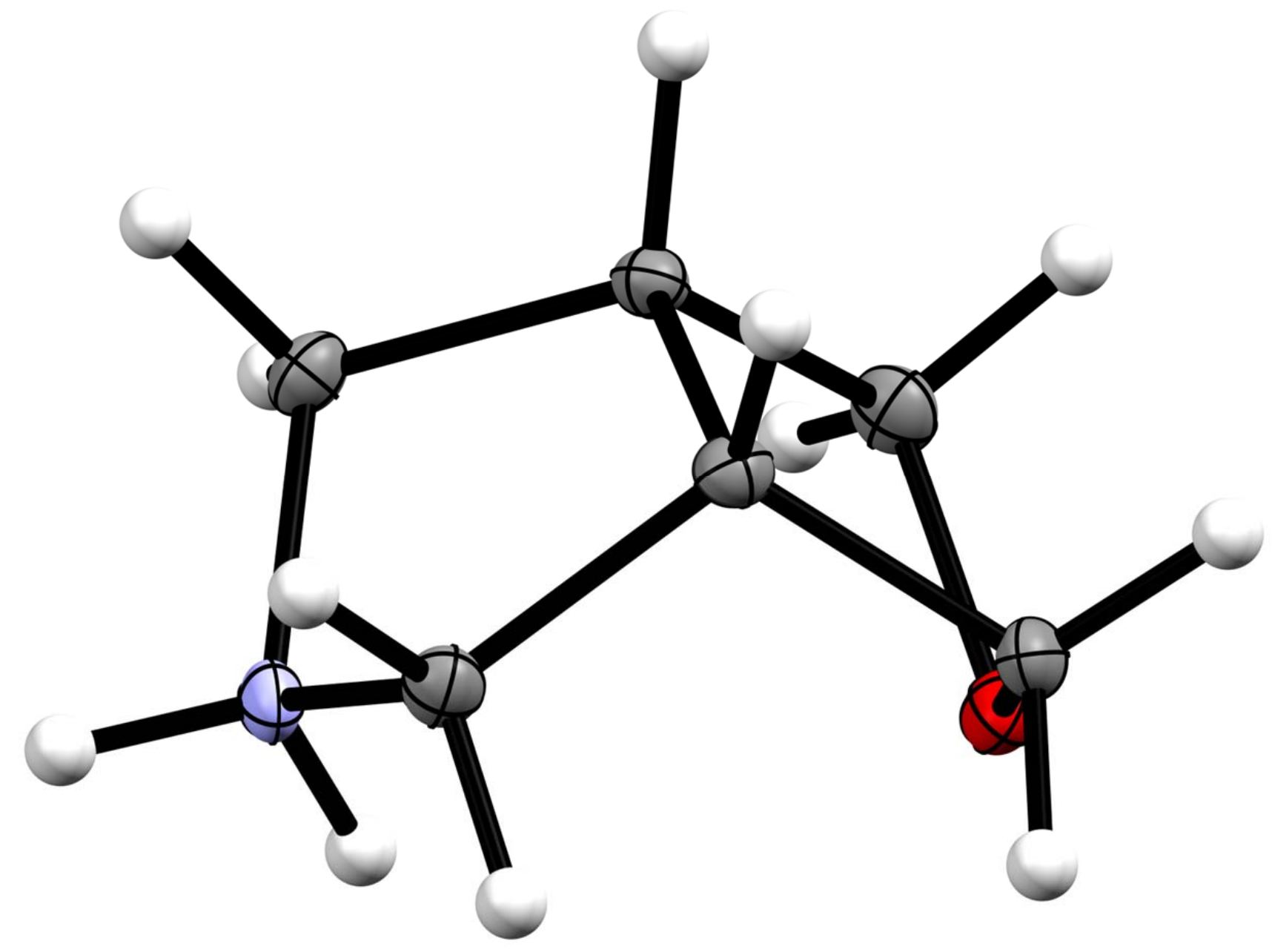

cis-Hexahydro-1H-furo[3,4-c]pyrrol-5-ium chloride (64) ORTEP diagram (Projection 1)

(Thermal ellipsoids are shown at $30 \%$ probability level) 


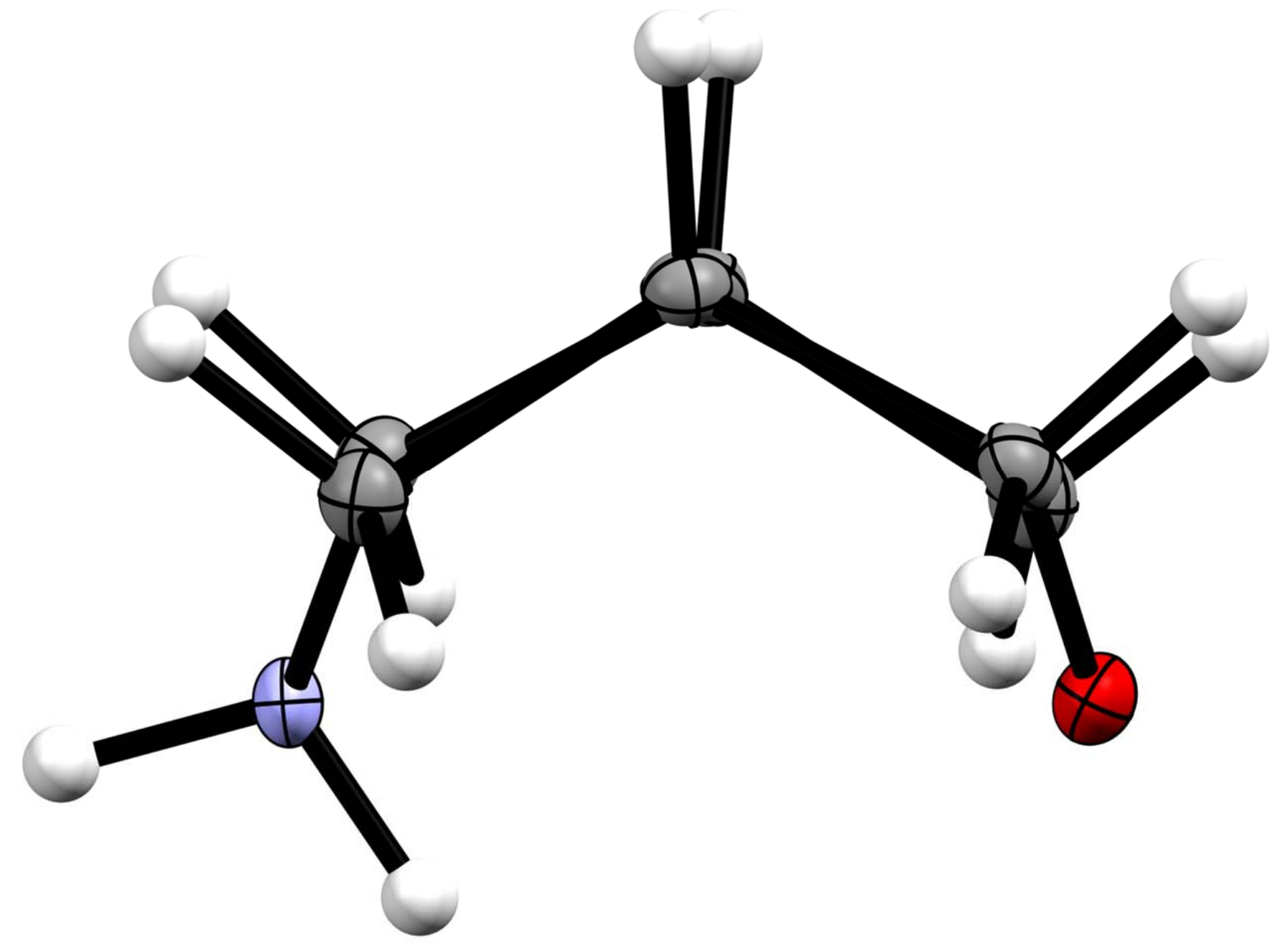

cis-Hexahydro-1H-furo[3,4-c]pyrrol-5-ium chloride (64) ORTEP diagram (Projection 2)

(Thermal ellipsoids are shown at $30 \%$ probability level) 
Table S1. Crystallographic data and experimental parameters for the compounds 14-16, 18, and 64.

\begin{tabular}{|c|c|c|c|c|c|}
\hline Parameter & 14 & 15 & 16 & 18 & 64 \\
\hline \multicolumn{6}{|l|}{ Unit cell dimensions } \\
\hline$a, \AA$ & $24.981(5)$ & $7.468(3)$ & $13.372(3)$ & $7.1176(10)$ & $6.9635(2)$ \\
\hline$b, \AA$ & 7.1333(13) & $10.606(6)$ & $5.9409(15)$ & $15.208(2)$ & $6.8977(2)$ \\
\hline$c, \AA$ & $12.150(2)$ & $12.627(4)$ & $12.492(3)$ & $10.1283(14)$ & $14.8768(4)$ \\
\hline$\alpha,{ }^{\circ}$ & 90 & 90 & 90 & 90 & 90 \\
\hline$\beta, \circ$ & 90 & 90 & $116.478(5)$ & $91.197(4)$ & $95.7031(18)$ \\
\hline$\gamma,{ }^{\circ}$ & 90 & 90 & 90 & 90 & 90 \\
\hline$V, \AA^{3}$ & $2165.0(7)$ & $1000.1(8)$ & $888.3(4)$ & 1096.1(3) & $711.03(3)$ \\
\hline $\mathrm{F}(000)$ & 880 & 408 & 376 & 520 & 320 \\
\hline Crystal system & Orthorhombic & Orthorhombic & Monoclinic & Monoclinic & Monoclinic \\
\hline Space group & Pna $2_{1}$ & $\mathrm{P} 2_{1} 2_{1} 2_{1}$ & $\mathrm{P} 2{ }_{1} / \mathrm{c}$ & $\mathrm{P} 2_{1} / \mathrm{c}$ & $\mathrm{P} 2_{1} / \mathrm{c}$ \\
\hline$Z$ & 8 & 4 & 4 & 4 & 4 \\
\hline$\mu, \mathrm{mm}^{-1}$ & 0.316 & 0.337 & 0.374 & 0.571 & 0.454 \\
\hline$d_{\text {calc }}, \mathrm{g} / \mathrm{cm}^{3}$ & 1.250 & 1.260 & 1.313 & 1.486 & 1.398 \\
\hline $2 \Theta_{\max }, \operatorname{grad}$ & 60 & 60 & 60 & 60 & 60 \\
\hline Measured reflections & 23892 & 7486 & 14085 & 11338 & 9573 \\
\hline $\mathrm{F}>4 \sigma(\mathrm{F})$ reflections & 2709 & 1127 & 1530 & 1867 & 1209 \\
\hline Parameters & 235 & 109 & 100 & 135 & 82 \\
\hline$R_{1}$ & 0.0677 & 0.0405 & 0.0359 & 0.0304 & 0.0392 \\
\hline$w R_{2}$ & 0.196 & 0.101 & 0.0936 & 0.0782 & 0.0932 \\
\hline$S$ & 1.05 & 1.06 & 1.036 & 1.063 & 1.087 \\
\hline CCDC number & 1943269 & 1943268 & 1943267 & 1943266 & 1943270 \\
\hline
\end{tabular}

